\title{
Scattering of Coastal-Trapped Waves by Irregularities in Coastline and Topography
}

\author{
by \\ John Lewis Wilkin \\ B.E.(Hons), University of Auckland (1982) \\ S.M., Massachusetts Institute of Technology (1985) \\ Submitted in partial fulfillment of the \\ requirements for the degree of \\ Doctor of Philosophy \\ at the \\ MASSACHUSETTS INSTITUTE OF TECHNOLOGY \\ and the \\ WOODS HOLE OCEANOGRAPHIC INSTITUTION
}

September 1988

(C) John L. Wilkin, 1988

The author hereby grants to MIT and to WHOI permission to reproduce and to distribute copies of this thesis document in whole or in part.

Signature of Author

Joint Program in Oefanographic Engineering Massachuset Institute of Technology

Woods Hole Oceanographic Institution August 9, 1988

Certified by

David C. Chapman

Associate Scientist, Woods Hole Oceanographic Institution Thesis Sџpervisor

Accepted by

W. Kendall Melville Chairman, Joint Committee for Oceanographic Engineering 


\title{
Scattering of Coastal-Trapped Waves by Irregularities in Coastline and
}

\author{
Topography \\ by \\ John Lewis Wilkin
}

\author{
Submitted to the Massachusetts Institute of Technology- \\ Woods Hole Oceanographic Institution \\ Joint Program in Oceanographic Engineering \\ on August 10, 1988, in partial fulfillment of the \\ requirements for the degree of \\ Doctor of Philosophy
}

\begin{abstract}
A study is conducted of the scattering of freely-propagating subinertial frequency coastaltrapped waves (CTWs) by large variations in coastline and topography using analytical and numerical techniques. Particular attention is paid to the role of stratification because, as shown, the introduction of even modest stratification can eliminate backscattered free-waves with large wavenumbers which occur, theoretically, in a barotropic ocean.

An analytical solution is presented for the scattering of barotropic waves incident upon a discontinuity in shelf width. Discussion of solutions relying on backscattered free-waves is avoided by considering only the range of parameters over which energy transmission is nearly $100 \%$. The solution shows there is a substantial transfer of energy to modes other than that of the incident wave. The transmitted mode most readily excited is that which has the acrossshelf structure most closely coinciding with that of the incident wave. For a widening shelf, energy is therefore readily transferred to higher modes. The resultant presence of multiple modes produces a strong modulation in flow intensity and phase progression downstream of the scattering region which may affect the interpretation of shelf wave observations. A nondispersive shelf wave 'pulse' of limited alongshelf extent scatters into a train of similarly shaped waves of all allowable modes, each propagating at its own free-wave speed.

To overcome limitations of the analytical study a numerical model which accomodates arbitrary density stratification, bathymetry, and coastline, is employed. Numerical simulations are conducted of the scattering of CTws by a set of topographic and coastline variations which are representative of many continental shelves. The strength of the scattering observed is found to be proportional to a topographic warp factor which estimates the severity of the topographic irregularities. The scattering is amplified by density stratification. A comparison of the effects of widening and narrowing topographies shows that the gross scattering effects of 'reciprocal' topographies are quite similar. Within the scattering region itself, the strengths of the scattered-wave-induced currents exhibit substantial variation over short spatial scales. On both widening and narrowing shelves, there is generally a marked intensification of the flow within the scattering region, and significant differences in the directions of the currents at points separated by a few tens of kilometers indicate the occurence of rapid variations in phase. On narrowing shelves, the influence of the scattering can extend upstream into the region of
\end{abstract}


uniform topography even when no freely-propagating backscattered waves exist.

A simulation is conducted of CTW scattering at a site on the East Coast of Australia where observations suggest the presence of scattered freely-propagating CTWs. The success of the model simulation in reproducing features of observations confirms that realistic shelf geometries can scatter significant levels of CTW energy, and that the scattered waves can have an appreciable signal in current-meter observations made on the continental shelf. This demonstrates that along irregular coastlines it is necessary to account for the possibility that CTW scattering processes may be in effect if oceanographic observations are to be interpreted correctly.

Thesis Supervisor: David C. Chapman

Title: Associate Scientist, Woods Hole Oceanographic Institution 


\section{Acknowledgements}

My very sincere thanks go to my advisor, Dave Chapman, for his consistent encouragement, enthusiasm, guidance, friendship and good humour throughout our collaboration. I could not have hoped for a better advisor.

I am also grateful to the other members of my committee: Ole Madsen, Bob Beardsley, Ken Melville, Ken Brink and Dale Haidvogel. In particular, I thank Dale for developing the PEM, and for answering all my questions about how it works, and Ken Brink for his interest, encouragement, and helpful discussions throughout this project.

This research was supported by the National Science Foundation under grants OCE8417769 and OCE85-21837, and by the WHOI Education Program. Computer facilities at the National Center for Atmospheric Research, which is funded by NSF, were used for the numerical experiments. The numerical work could never have been completed without the assistance of the consultants at NCAR, and Andy Maffei at WHOI who never tired of me asking why the network was down.

I have thoroughly enjoyed my time in the MIT/WHOI Joint Program, due to the friendship of way too many people to mention here. But special thanks go to Harry, Paul, Maggie, Rich and Teddi. To everyone in Bigelow, in particular the Tuesday/Thursday donut crew - thanks. The students of the PO Department adopted me as one of their own for which I am very grateful. And for instructing me in the finer points? of American sports I thank the OE softball team and everyone at Sunday touch football. Thanks to everyone at the WHOI Education Office, and at MIT a big thanks to Mary. However, no thanks to anyone for all the sheep jokes.

To Carol, my thanks for being such a great housemate and a wonderful friend, and for helping to cultivate my culinary skills. I'll miss you. To my compatriots Ross, Hamish and Peter, thanks for helping me retain some vestige of my accent. And thanks Mum and Dad for understanding that this is what I really wanted to do.

A very special thanks, and a big hug, goes to Liz — for her support and love, and for holding me together during the crunch (check out Figure 6-1!).

And to everyone I forgot to mention, 'thankyou for your support.' 


\section{Contents}

$\begin{array}{lll}1 & \text { Introduction } & 7\end{array}$

2 Background $\quad 12$

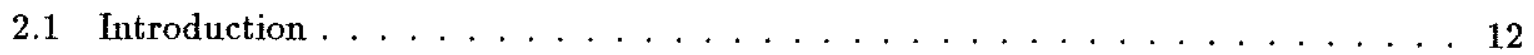

2.2 . Slow Alongshelf Variations . . . . . . . . . . . . . . . . . . . 13

2.3 Small Variations $\ldots \ldots \ldots \ldots \ldots \ldots$

2.4 Small Random Variations . . . . . . . . . . . . . . . . . . . . 14

2.5 Larger Variations . . . . . . . . . . . . . . . . . . . . 15

2.6 Abrupt Variations . . . . . . . . . . . . . . . . . . 17

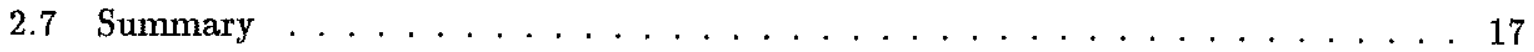

3 Analytical Model and Results: Barotropic Shelf Wave Scattering at a Discontinuity in Shelf Width 19

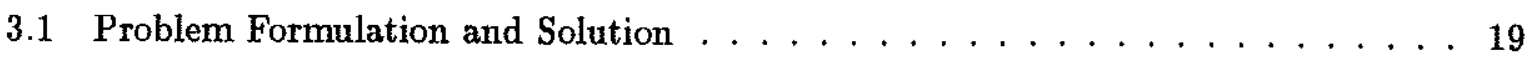

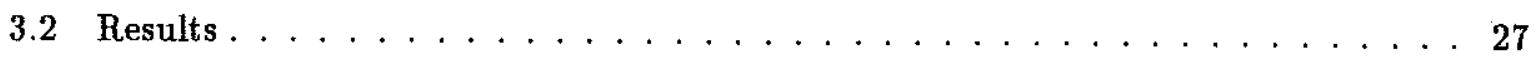

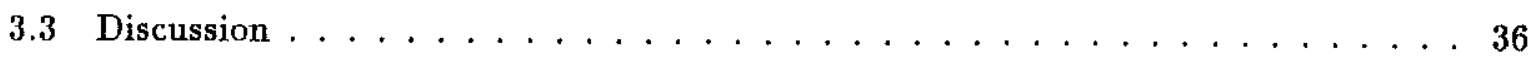

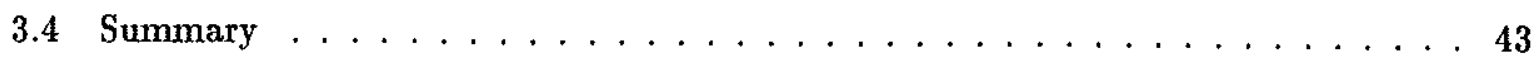

4 The Numerical Model $\quad \mathbf{4 5}$

4.1 Introduction . . . . . . . . . . . . . . . . . 45

4.2 The Haidvogel Primitive Equation Model $\ldots \ldots \ldots$

4.3 Orthogonal Curvilinear Coordinates . . . . . . . . . . . . . 48

4.4 Coastal-Trapped Wave Across-Shelf Modal Structures . . . . . . . . . . . . 49 
4.5 The Numerical Wavetank . . . . . . . . . . . . . . . . . . 51

4.6 Analysis: Modal Decomposition . . . . . . . . . . . . . . . . 53

4.7 Model Performance Test: Comparison with Analytical Results . . . . . . . . . . 54

5 Numerical Results and Discussion $\quad 58$

5.1 CTW Dispersion Properties and Scattering Regimes . . . . . . . . . . 58

5.2 Narrowing Shelf Results . . . . . . . . . . . . . . . . . . 63

5.3 Widening Shelf Results . . . . . . . . . . . . . . . . . . 74

5.4 Flow Field Near the Scattering Region . . . . . . . . . . . . . . . 79

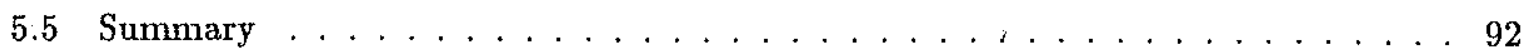

6 Observations of Coastal-Trapped Wave Scattering: A Case Study 95

7 Summary 111

Appendix A Scattering of a Barotropic Shelf Wave Pulse 114 


\section{Chapter 1}

\section{Introduction}

Over the past twenty years, oceanographic observations have revealed that patterns of sea-level and current fluctuations with periods of a day or more propagate along the continental shelves of many of the world's oceans. These subinertial-frequency wavelike motions, which extend across the width of the shelf and are often generated by winds many hundreds of kilometers from where they are observed, account for a major part of the large-scale, low-frequency current and sea-level variability along many coastlines. Lagged-correlation and cross-spectral analyses of oceanographic and meterological data show that patterns in sea-level and current fluctuations travel along the Pacific coasts of the United States (Halliwell and Allen, 1984), Mexico (Enfield and Allen, 1983) and Peru (Smith, 1978; Brink, 1982b) at speeds of typically a few hundred kilometers per day. An example of this propagation is shown in Figure 1-1 where it can be seen that the pattern of fluctuations in sea-level observed at San Juan, Peru $\left(15^{\circ} 20^{\prime} \mathrm{S}\right)$ during May-July, 1976 was very similar to that observed two days previously at Callao, $400 \mathrm{~km}$ to the north (Smith, 1978).

Theoretical studies have indicated that wave motions with subinertial frequencies propagating along continental shelves can be supported by two distinct physical characteristics of a coastal ocean. When considered separately, the properties of density stratification and acrossshelf sloping topography support the two classes of waves generally referred to as internal (or baroclinic) Kelvin waves, and barotropic shelf waves, respectively. In an ocean of coustant depth with a vertical coast, an infinite set of internal Kelvin wave (IKW) modes exists. IKWs are essentially internal gravity waves modified by the Earth's rotation. The lowest mode is 

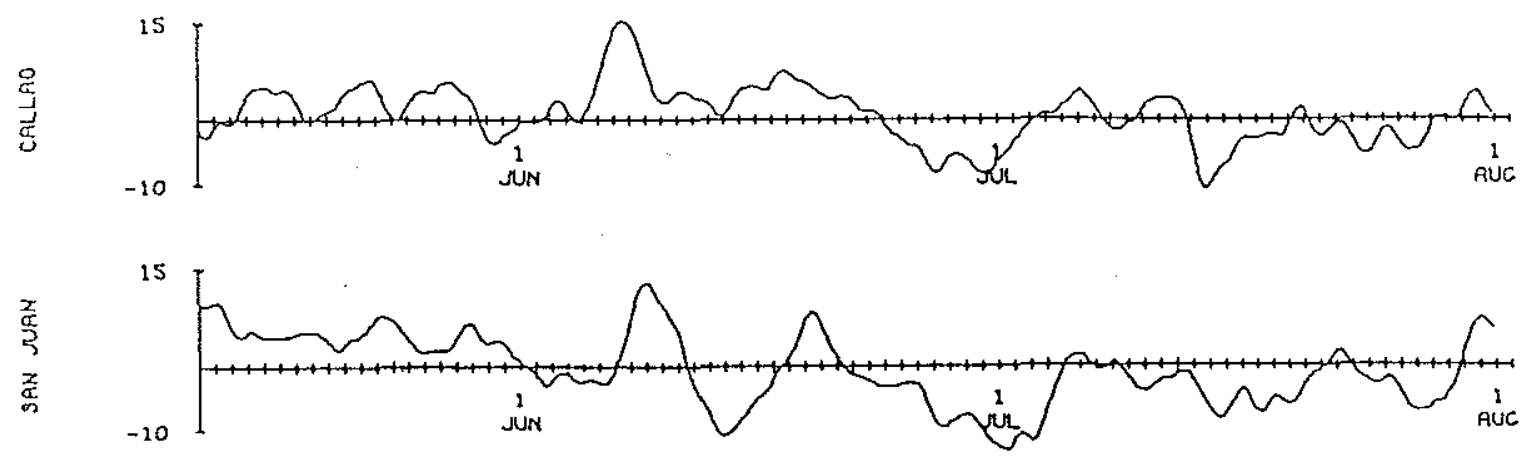

Figure 1-1: Low-pass filtered adjusted sea-level records in $\mathrm{cm}$ from Callao $\left(12^{\circ} 04^{\prime} S\right)$ and San Juan $\left(15^{\circ} 20^{\prime} S\right)$, Peru, during May-July 1976 (from Smith, 1978).

trapped against the coastal wall with an exponential offshore decay scale of the first internal Rossby radius of deformation. Higher modes are trapped more closely to the wall, propagate more slowly, and have more zero crossings in their vertical structure than lower modes. Alongshore propagation is always cyclonic with respect to the deep sea, i.e. with the coast on the right (left) in the northern (southern) hemisphere. The presence of sloping bottom topography in the absence of stratification admits the second class of wave motions, barotropic shelf waves (BSws). These are trapped against the coast with an offshore decay scale comparable to that of the topography and are supported by a potential vorticity restoring mechanism (e.g. Gill, 1982; p.411). The number of zero crossings in the across-shelf structures of BSWs increases with mode number. Unlike internal Kelvin waves, barotropic shelf waves are dispersive and are limited to a finite subinertial frequency range above which they cannot propagate. At low wavenumbers (long wavelengths), the group velocity of Bsws is cyclonic with respect to the deep sea but as wavenumber increases, the group velocity decreases eventually passing through zero, provided the bottom slope is bounded, to a region of short waves with group velocity in the opposite sense (Hutlinance, 1975). 
In a typical coastal ocean both stratification and topography are present. As a result the subinertial-frequency waves observed on many continental shelves are a hybrid class of waves, generally termed coastal-trapped waves (cTWs), having characteristics of both Kelvin and shelf waves. In actuality, IKWs and BSWs are not distinct sets of waves but rather they are the high and low stratification limits, respectively, of hybrid coastal-trapped waves. Huthnance (1978) showed that increasing stratification increases the frequency of CTws at all wavenumbers. This property, which is discussed in detail in section 5.1, can cause a qualitative alteration in the behavior of cTws at small wavelengths when the stratification is sufficiently strong to cause the dispersion curves to increase monotonically to the Coriolis frequency $f$. In this case, each mode can occur at any subinertial frequency but is limited in wavenumber. There are then no zero group velocity points and, consequently, phase and energy always propagate in the same direction. Chapman (1983) has demonstrated that such a wavenumber-limited regime generally occurs on shelves where $N h_{0} / f L>1$, where $N$ is the maximum buoyancy frequency, $h_{0}$ the deep-sea depth and $L$ the shelf-slope width. Furthermore, computations of free-wave dispersion curves using observed geometry and stratification for many shelves, including the Pacific Northwest (Battisti and Hickey, 1984) and Peru (Brink, 1982b), suggest that wavenumber-limited free waves are more nearly the rule than the exception.

Statistical analyses of sea-level, current and wind data combined with models of CTw dynamics along the West Coast of North America (Halliwell and Allen, 1987) and the East Australian coast (Freeland et al., 1986; Church et al., 1986a) confirm that the waves observed there have speeds and across-shelf structures consistent with those expected from theoretical analyses. Further validation of CTW theory is provided by so-called "first-order wave equation" (FOWE) studies. The FOWE method was first proposed by Gill and Clarke (1974) and Gill and Schumann (1974) and has since been generalized by Clarke (1977a), Brink and Allen (1978), Brink (1982a) and Clarke and Van Gorder (1986). If only low frequencies (periods in the range several days to a few weeks) are considered, and it can be assumed that alongshelf variations in the currents occur over distances much greater than the shelf width, then the propagation of a set of CTws is governed by a set of coupled first-order wave equations. (The combined assumptions of low-frequency and large alongshelf scale are frequently termed the "long-wave approximation".) In applications of the Fowe method, observed alongshelf winds are assumed 
to generate a set of long CTW modes and these are propagated along the coast according to the theory with variations in bottom topography and coastline shape being incorporated only parametrically through slow alongshelf changes in the long-wave phase speeds, wind-coupling coefficients and bottom-friction coefficients. Along relatively straight coasts, this Fowe approach has been quite successful. The studies of Battisti and Hickey (1984) using a single CTw mode, Church et al. (1986b) and Chapman (1987) using up to three modes, and Mitchum and Clarke (1986) using four to seven modes obtained generally good agreement between observed and hindcast sea-level and current fluctuations.

However, most coastlines vary significantly over spatial scales much shorter than cTw length scales. This introduces the possibility that appreciable scattering of CTW energy from one mode into others can occur over alongshelf distances comparable to the shelf width; a process not accounted for in FOWE analyses. Indeed, the poorest agreement between observations and the time series hindcast by Mitchum and Clarke (1986) occurs at the northern end of the West Florida shelf where the shelf narrows abruptly. Chapman (1987) found that the propagation of CTws through the irregular geometry south of Point Conception, California, where the shelf widens abruptly, the coastline bends sharply and the coastal waveguide is split by a chain of offshore islands, was not modelled adequately by FOWE dynamics. CTW signals observed south of the Gulf of California were found to be uncorrelated with sea-level fluctuations on either side of Baja California (Enfield and Allen, 1983) and observations by Griffin and Middleton (1986) suggest the possible generation of high mode CTws in the lee of Fraser Island, Australia. While likely to have a significant effect on the propagation of CTWs, scattering processes at places where the shelf width and topography change sharply are not yet well understood.

To date, with few exceptions, analytical studies of CTW scattering have been limited to the scattering of Kelvin waves by small or abrupt coastline variations in a uniformly stratified ocean without coastal topography, and to the scattering of barotropic shelf waves by slowly varying, small or randomly placed small changes in topography and coastline. While relevant to coasts at very low latitudes or with unusually strong stratification, Kelvin wave studies ignore the presence of coastal topography and therefore do not aid in improving understanding of the signature of CTW scattering in the shallow waters of the continental shelf. Barotropic shelf wave studies provide more insight into behavior on the shelf but few have considered scattering by 
large variations in coastline or topography and most studies have tended to emphasize results indicating scattering into short reflected Bsws or the resonance of modes with zero group velocity. It was noted earlier that the introduction of modest stratification can qualitatively alter the dispersion characteristics of BSws by eliminating zero group velocity points and short BSWs with opposite group velocity. This has important consequences when considering the scattering of CTws because it eliminates the possibility of reflection or backscattering of CTW energy and suggests that the neglect of stratification in BSW scattering studies could constitute a significant omission.

The objective of this study has been to investigate the scattering of CTWs by large variations in coastline and topography occuring over alongshelf distances comparable to the shelf width in a realistically stratified coastal ocean. The results of the study fall into two categories: analytical and numerical. Following a review of previous studies in Chapter 2, an analytical solution for the scattering of barotropic shelf waves at a discontinuity in shelf width is presented in Chapter 3. These analytical results answer some questions and pose others. They indicate the likely strength of scattering by large coastline variations yet skirt the issue of the role of stratification by limiting discussion to the range of parameters over which the solution shows that reflected short barotropic shelf waves, which may not be present in a realistically stratified ocean, are not generated. The most unsatisfactory result of this limitation is the exclusion of any discussion of narrowing shelves. Only through the use of the mumerical model described in Chapter 4 has a treatment of the full problem including stratification and arbitrarily varying topography and coastline been possible. In Chapter 5, results are presented from numerical simulations of CTW scattering by a set of topographic and coastline variations which are representative of many continental shelves. It is found that both the strength of the scattering induced by the topographic variations and the effects of stratification are related to CTW dispersion characteristics. Finally, in Chapter 6, the understanding of CTW scattering processes gained from the study is applied to the interpretation of a simulation of CTW scattering at a site on the East Coast of Australia where observations made by Griffin and Middleton (1986) suggest the presence of scattered freely-propagating CTWs. 


\section{Chapter 2}

\section{Background}

\subsection{Introduction}

The purpose of this Chapter is to review theoretical and numerical studies of relevance to the problem of CTw scattering. A detailed analysis of the properties affecting CTW energetics and dispersion characteristics may be found in Huthnance (1978), and for reviews of CTw observations and studies of the generation and propagation of CTws along straight coasts the reader is referred to Clarke (1977a), Mysak (1980a,b), Allen (1980) and Huthnance et al. (1986).

A general study of CTW scattering when both topography and stratification are present has so far proven analytically intractable. Nevertheless, several useful results have been obtained from analyses of barotropic shelf wave scattering by slow or small alongshelf variations and Kelvin wave scattering by abrupt coastline changes. Most of the latter studies concern the propagation of barotropic Kelvin waves but apply equally to internal Kelvin waves (IKws) since an internal Kelvin wave on the interface between two fluids of different density behaves exactly as a barotropic Kelvin wave with the gravitational acceleration reduced by the relative density difference of the layers. In a continuously stratified ocean multiple IKW modes exist but these remain uncoupled provided the ocean depth is constant. 


\subsection{Slow Alongshelf Variations}

Alongshelf variations occurring over distances greater than typical CTW wavelengths will not directly scatter CTW energy from one mode to another but can still slowly modify CTW waveforms. Dorr and Grimshaw (1986) considered the effect of variation of the Coriolis parameter (f) with latitude on barotropic shelf waves and found that the wave amplitude varies so as to conserve alongshelf energy flux. For waves with periods of roughly 2-10 days, the wave elevation varies as $f^{1 / 2}$. At longer periods, Rossby wave radiation to the deep sea can occur, particularly at high latitudes, causing damping of the shelf waves. However, the damping rate is low and less than that expected from bottom friction.

Miles (1972) showed that Kelvin wave amplitudes vary as $f^{1 / 2}$ also, and that Kelvin waves become more closely trapped to the coast as they propagate poleward. The phase speeds of both Kelvin waves (Miles, 1972; Clarke, 1977b) and barotropic shelf waves (Grimshaw, 1977) are increased around weakly curving capes and decreased around bays.

\subsection{Small Variations}

Studies of small variations in the coastal waveguide are generally conducted as perturbation analyses. Consequently, the scattered wave amplitudes are of the order of the small perturbation parameter and do not appreciably alter the total wave field. Nevertheless, such studies are useful for suggesting the likely relative importance of variations in waveguide parameters. The scattering of an internal Kelvin wave by a small ridge perpendicular to the coast was considered by Killworth (1978). All other possible IKW modes are excited and the scattered field for each mode consists of two contributions: a steady flow over the ridge and a propagating wave. Most scattered energy goes into the mode next highest from the incident mode.

Buchwald (1977) considered the scattering of barotropic shelf waves on an exponential shelf by a small perturbation to an otherwise straight coast. All possible transmitted and reflected modes are generated and the strength of the scattering increases with frequency. If the frequency is near that at which one of the scattered modes has zero group speed, a resonant response occurs. A similar study by Chao et al. (1979) considered Bsw scattering by a small, isolated (delta-function) bump in the topography. Scattering is strongest for high incoming modes and 
for a bump near the coast. The propagating scattered wave with the highest mode number is the most readily excited. As frequency increases, the amount of energy scattered forward increases while back-scattering decreases with, again, the possibility of resonance at zero group speed frequencies. Chao et al. (1979) note that while their results predict a cascade of energy to high modes and wavenumbers, integration of the solution over an extended topographic feature leads to self-cancelation of high-wavenumber waves.

Allen (1976) examined a slow variation in shelf width and topography occuring over a distance much greater than the shelf width. (This scaling is the longwave approximation used in FOWE analyses; it eliminates short reflected BSWs.) The lowest order free shelf wave has a cross-shelf structure and speed dictated by the local shelf geometry. In the region of slowly varying topography, disturbances in the other modes are generated. If $L_{I}$ is the length of the incident wave and $L_{B}$ the distance over which the topography varies, then the loss of energy from the incident wave due to scattering is comparatively smaller when $L_{I} \gg L_{B}$ than when $L_{B} \gg L_{I}$. (Note that $L_{B}$ is always much greater than the shelf width.) For $L_{B} \gg L_{I}$ the high modes lose relatively more energy than the lower modes. Brink (1986) also made the longwave approximation and obtained a solution for the scattering of long Crws by a small bottom irregularity on a shelf with both topography and stratification. The loss of energy from the incident wave to scattered waves is comparable to typical losses due to bottom friction. Brink (1986) found no clear trend in the changes in scattered mode amplitudes with changing stratification.

\subsection{Small Random Variations}

Kelvin wave propagation along a coast which is straight except for small random deviations was considered by Mysak and Tang (1974). The coastal variations reduce both the phase speed and energy flux of the coherent wave. Brink (1980) examined Bsws propagating in a channel with randomly perturbed exponential bottom topography. The small bottom bumps cause a modification to the coherent wave dispersion relation which is dominated by peaks centered at wavenumbers where the scattered modes have zero group speed. Scattering into propagating modes causes damping of the coherent wave comparable in strength to that expected from bottom friction. The frequency dependence of the damping creates frequency "pass bands" for 
free BSWs. Brink (1980) notes that the inclusion of a mean current can eliminate zero group speed points in the dispersion relation of the scattered modes, thereby reducing topographic damping. This suggests that stratification, which can also eliminate zero group speed points, will likely diminish the strength of topographic damping.

\subsection{Larger Variations}

Hsueh (1980) showed that large variations in shelf width occurring over large alongshelf distances (i.e., where the longwave approximation is valid) will not scatter BSws if the topography varies in a "shelf-similar" manner where the distance from each isobath to the coast remains a fixed fraction of the local shelf width. Hsueh (1980) proceeded to consider scattering due to small deviations from shelf-similarity, identifying that scattering could create cross-shelf phase differences in flow events comparable to the phase differences observed by Brink and Allen (1978) which were attributed to bottom friction effects. Davis (1983) showed that the restrictive longwave approximation need not be made in the definition of shelf-similar topographies. Using conformal mapping arguments he demonstrated that any shelf topography can be mapped to one of constant width in the new mapped coordinate, and that the equation governing the propagation of dispersive Bsws is invariant under such a mapping. As a result, the two topographies are geometrically similar (Davis calls them 'shelf-similar' which tends to confuse the issue) in the sense that the depth variations of the two topographies will scatter BSWs in the same manner. Furthermore, Davis showed that if the logarithm of the depth satisifies Laplace's equation then the shelf maps to a shelf of constant width with parallel isobaths which therefore will not scatter BSws. This property is the foundation of Hsueh's definition of shelf-similarity. Strictly speaking, Hsueh's definition is not valid for a shelf which changes width over an alongshelf distance comparable to the shelf width and Davis's more rigorous definition, which requires the evaluation of a conformal transformation to map the coast and offshore limit of the shelf to straight lines, should be applied. However, inspection of Davis's example (Davis, 1983, Figure 1) in which the shelf width changes by a factor of 1.5 over a distance twice the mean shelf width, it is clear that the definitions are almost identical. In the longwave limit the two definitions coincide. It should be stressed that this property holds only for barotropic shelves. With the introduction of stratification the governing equation is no longer invariant 
under conformal transformations and the possibility of CTW scattering cannot be ruled out.

Topography which is shelf-similar in the sense defined by Hsueh (1980) but varying over an alongshelf distance comparable to the shelf width was considered by Webster (1987). The scattering of Bsws is weak unless the relative change in shelf width is large and the length of the shelf width transition zone is less than the shelf width. Webster (1987) attributed this scattering to shelf wave dispersion at the high frequencies considered. However, the analysis by Davis (1983) suggests that the scattering may be due to a departure from truly shelf-similar topography and raises a question as to whether a change of coordinates might alter Webster's (1987) results.

The scattering of a BSW by variable topography on a shelf of constant width was considered by Wang (1980). Assuming a time periodic solution Wang solved numerically for the phase and amplitude of the mass transport streamfunction. The topographies studied included examples of a canyon, a ridge, and converging or diverging depth contours where, in the direction of wave propagation, isobaths moved shoreward or seaward, respectively. These topographic variations occurred over an alongshelf distance equal to the shelf width. In general, for the cases of convergence or divergence of depth contours, little backscattering occurs. However, the reflected energy flux increases as frequency increases and for converging isobaths it is possible for the incident wave frequency to exceed the maximum possible frequency of all outgoing modes, in which case total reflection results. No conclusion about scattering into multiple modes forward of a convergence was made because the analysis was limited to high frequencies at which only a single mode could propagate. (Note that the across-shelf resolution of Wang's (1980) model, 8 grid points, would not have been sufficient to resolve the many zero crossings in higher mode structures.) Wang (1980) did consider a divergent topography at sufficiently low frequency that two transmitted modes propagated. For a mode 1 incident wave more than $90 \%$ of the energy flux remained in mode 1 . The canyon and ridge topographies both backscatter a large portion of the incoming wave energy. They also significantly alter the phase progression of the wave immediately over the varying topography. However, when averaged across the ridge or canyon, the phase progression is nearly constant. This suggests that the presence of a ridge or canyon may not be apparent in phase changes estimated from observations made on either side of the ridge or canyon. 


\subsection{Abrupt Variations}

A Kelvin wave incident upon a right angle corner in the coastline was considered by Buchwald (1968). At subinertial frequencies the Kelvin wave turns the corner without change of amplitude. Packham and Williams (1968) deduced that this result holds for all angles of the coastline bend.

Using principles of geometrical optics, Davis (1981) obtained solutions for the scattering of a BSW on an exponential shelf due to a semicircular headland, and a slender peninsula perpendicular to the coast.

\subsection{Summary}

In a barotropic coastal ocean, scattering into all possible BSw modes occurs. Short reflected waves are readily generated and resonance at frequencies where scattered modes have zero group speed is possible. This resonance can trap motions over topographic features and can lead to damping of the incident wave. However, as noted in Chapter 1, and demonstrated in detail is section 5.1 , modest stratification can eliminate propagating reflected waves and may qualitatively alter these barotropic scattering results.

A class of shelf-similar topographies has been identified for which the dispersion relation of BSWs does not vary with alongshelf coordinate. BSWs propagate through these changing topographies without scattering. Dispersion properties also figure in Kelvin wave scattering studies. In an ocean of constant depth there is no coupling between internal Kelvin wave modes and, at subinertial frequencies, no class of wave motions with opposing group velocity. As a result, a subinertial frequency Kelvin wave encountering abrupt changes in coastline direction cannot scatter into any other waves and therefore emerges from the coastal irregularity without loss of amplitude. If the depth is perturbed slightly there is scattering into all other possible vertical modes.

With the exception of Wang's (1980) numerical study, no studies to date have considered large variations in coastline or topography which might scatter appreciable amounts of energy into multiple CTW modes. Furthermore, neither the effect that scattering might have on the interpretation of CTW observations, nor the likely role of stratification have been discussed. An initial step toward correcting this omission is taken in Chapter 3 where an analytical solu- 
tion is presented for the scattering of a BSW by a discontinuity in shelf width. Care is taken in Chapter 3 to avoid solutions where energy is scattered into barotropic short waves which would be substantially altered and might be altogether absent in a realistically stratified ocean. The succeeding chapters extend the study to include stratification and address more realistic topographic variations. 


\section{Chapter 3}

\section{Analytical Model and Results: Barotropic Shelf Wave Scattering at a Discontinuity in Shelf Width}

Previous analytical studies of barotropic shelf wave scattering have considered only small variations in bottom topography, small perturbations to a straight coast or slow alongshelf variations on a scale much longer than the shelf width. In this chapter, a theoretical investigation of the scattering of free barotropic shelf waves by an abrupt change in shelf width in a barotropic ocean is presented. (This chapter has been published as "Scattering of continental shelf waves at a discontinuity in shelf width" by J.L. Wilkin and D.C. Chapman in the Journal of Physical Oceanography (1987), volume 17, pp 713-724.)

\subsection{Problem Formulation and Solution}

An inviscid, barotropic, coastal ocean on a northern hemisphere $f$-plane is considered. Making the rigid-lid approximation, the linear shallow water equations become

$$
\begin{gathered}
u_{t}-f v=-g \eta_{x} \\
v_{t}+f u=-g \eta_{y} \\
(h u)_{x}+(h v)_{y}=0
\end{gathered}
$$


where $u$ and $v$ are the cross-shelf $(x)$ and alongshelf $(y)$ components of velocity respectively, $\eta$ is the surface height perturbation, $g$ the gravitational acceleration, $f$ the Coriolis parameter and $h$ the depth. Subscripts denote partial differentiation. Defining a transport streamfunction

$$
h u=-\psi_{y} ; h v=\psi_{x}
$$

(3.1) through (3.3) combine to yield a vorticity equation

$$
\left[\left(\frac{\psi_{y}}{h}\right)_{y}+\left(\frac{\psi_{x}}{h}\right)_{x}\right]_{t}-f\left[\left(\frac{\psi_{y}}{h}\right)_{x}-\left(\frac{\psi_{x}}{h}\right)_{y}\right]=0
$$

The depth is assumed to be independent of $y$, and to increase exponentially offshore:

$$
h(x)=h_{0} e^{-2 \lambda x}
$$

where $h_{0}$ is the depth at the outer edge of the shelf. For large values of $\lambda$, the shelf drops off steeply at its outer limit while for smaller values it is more uniformly and weakly sloping. Therefore, $\lambda$ will be referred to as the slope steepness. For a travelling wave with frequency $\omega$ and alongshelf wavenumber $\ell, \psi$ takes the form

$$
\psi=\phi(x) e^{i(\ell y-\omega t)}
$$

Substitution of (3.6) and (3.7) into (3.5) shows that the cross-shelf structure function $\phi(x)$ satisfies

$$
\phi_{x x}+2 \lambda \phi_{x}+\left(\frac{2 \lambda f \ell}{\omega}-\ell^{2}\right) \phi=0
$$

A straight coast is placed at $x=L$. The origin $x=0$ is the offshore edge of the shelf where a wall rather than an open boundary has been placed for analytical convenience. This retains the qualitative properties of the open boundary (Brink, 1980) while making the crossshelf modal structure independent of alongshelf wavenumber and allowing the use of a simple mode-matching method. The boundary conditions of no flow through the walls require that $\psi$ be constant along $x=0$ and $x=L$. To be consistent with $\ell \neq 0$, and the lack of a mean flow in the channel, the constant is chosen to be zero so that

$$
\phi(0)=\phi(L)=0
$$

Solving (3.8) subject to (3.9) yields

$$
\phi_{n}(x)=e^{-\lambda x} \sin k_{n} x
$$


where

$$
k_{n}=\frac{n \pi}{L} ; n=1,2,3 \ldots
$$

with $n$ being the mode number. The dispersion relation for mode $n$ is

$$
\omega=\frac{2 \lambda f \ell_{n}}{k_{n}^{2}+\lambda^{2}+\ell_{n}^{2}}
$$

The $\phi_{n}$ satisfy an orthogonality condition

$$
\frac{2}{L} \int_{0}^{L} \phi_{n} e^{2 \lambda x} \phi_{m} d x=\delta_{m n}
$$

where $\delta_{m n}$ is the Kronecker delta. Were the wall to be replaced by the more realistic condition that the shelf abuts a semi-infinite flat-bottom ocean, the solution over the shelf would be matched to a solution in the deep ocean which decays exponentailly toward $x=-\infty$, and (3.11) would be replaced by (e.g. Buchwald and Adams, 1968)

$$
\tan k_{n} L=\frac{-k_{n}}{\lambda+\ell_{n}}
$$

The resultant effect on the free-wave dispersion curves is shown in Figure 3-1 for the case $\lambda L=2.25$. The presence of the offshore wall has no qualitative, and little quantitative, effect, so its use should not limit the applicability of the present results.

The scattering problem considered is that of a shelf wave travelling in a channel whose width changes abruptly from $L_{1}$ to $L_{2}$ at $y=0$ (see Figure 3-2). A wave of amplitude $\hat{A}$, wavenumber $\hat{\ell}$ and mode number $I$ is assumed to be incident upon the coastline bend from $y=-\infty$. The solution on either side of the bend may then be written

$$
\begin{gathered}
\psi_{-}=\hat{A} \phi_{1 I} e^{i(\hat{\ell} y-\omega t)}+\sum_{p=1}^{\infty} A_{p} \phi_{1 p} e^{i\left(\ell_{1} y-\omega t\right)}, y<0 \\
\psi_{+}=\sum_{q=1}^{\infty} B_{q} \phi_{2 q} e^{i\left(\ell_{2} y-\omega t\right)}, y>0
\end{gathered}
$$

where

$$
\begin{aligned}
& \phi_{1 p}=e^{-\lambda x} \sin k_{1 p} x ; \quad k_{1 p}=\frac{p \pi}{L_{1}} ; p=1,2,3 \ldots \\
& \phi_{2 q}=e^{-\lambda x} \sin k_{2 q} x ; \quad k_{2 q}=\frac{q \pi}{L_{2}} ; q=1,2,3 \ldots
\end{aligned}
$$

and $k_{1 p}, \ell_{1 p}$ and $k_{2 q}, \ell_{2 q}$ satisfy (3.12). The first subscript ( 1 or 2$)$ on $k, \ell$ and $\phi$ denotes the shelf width $\left(L_{1}\right.$ or $\left.L_{2}\right)$ to which the variable corresponds. The second subscript is the mode 


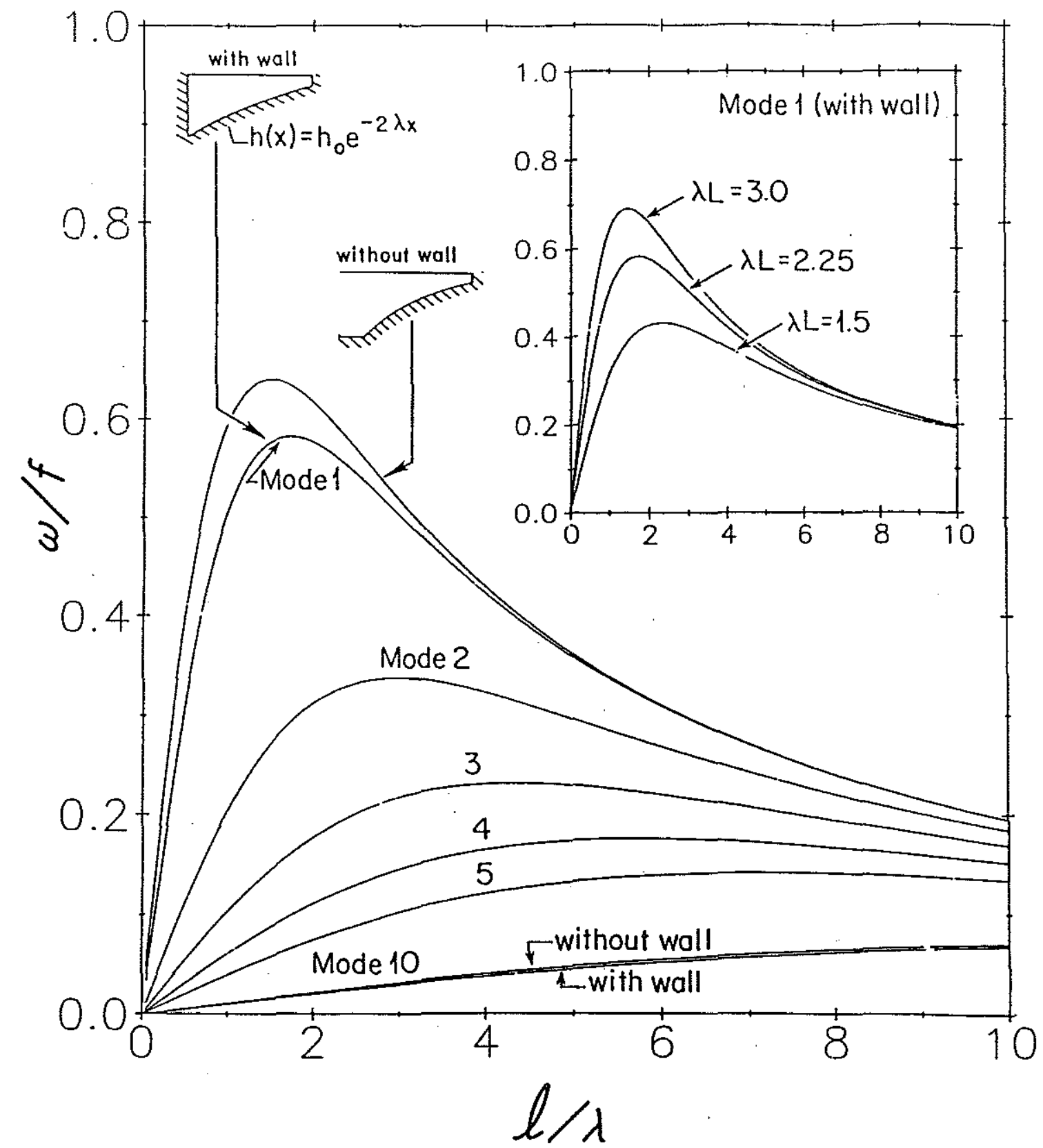

Figure 3-1: Barotropic shelf wave dispersion curves for a shelf with exponential depth profile for the case $\lambda L=2.25$. For modes 1 and 10 a comparison is made of two offshore boundary conditions. The remaining curves are computed with an offshore wall. Inset shows mode 1 dispersion curves for a range of $\lambda L$ values. 


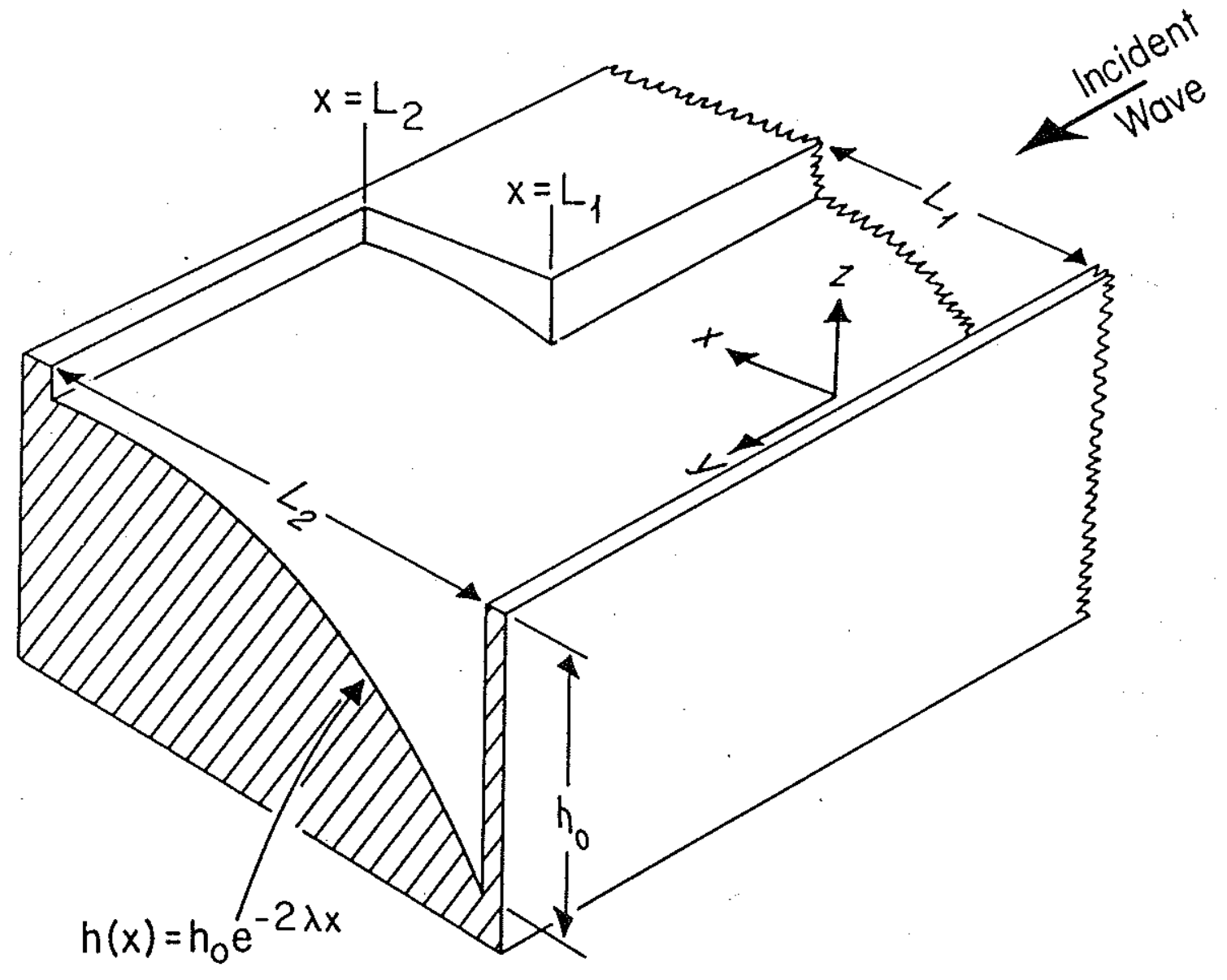

Figure 3-2: Problem geometry. 
number and corresponds to $n$ in equations (3.10) through (3.12). The dispersion relation (3.12) is a quadratic equation for $\ell_{n}$ with the solution

$$
\ell_{n}=\frac{f \lambda}{\omega} \pm\left[\left(\frac{f \lambda}{\omega}\right)^{2}-\left(k_{n}^{2}+\lambda^{2}\right)\right]^{1 / 2}
$$

If $(f \lambda / \omega)^{2}>k_{n}^{2}+\lambda^{2}$, then $\ell_{n}$ is real and mode $n$ propagates energy as a free wave. Since the $\ell_{1 p}$ represent reflected waves and the $\ell_{2 q}$ are transmitted waves, the radiation conditions require that if $\ell_{1 p}, \ell_{2 q}$ are real, they must correspond to negative, positive group , velocity respectively. This means that the $+(-)$ sign in (3.19) must be used to compute real $\ell_{1 p}\left(\ell_{2 q}\right)$. If $\ell_{1 p}, \ell_{2 q}$ are complex, i.e., $(f \lambda / \omega)^{2}<k_{n}^{2}+\lambda^{2}$, then the sign of the imaginary part must be selected to give an evanescent alongshelf structure trapped at the discontinuity (i.e. exponentially decaying away from $y=0)$. Thus the $-(+)$ sign must be used to compute complex $\ell_{1_{p}}\left(\ell_{2 q}\right)$.

The complex scattered wave amplitudes $A_{p}, B_{q}$ are determined by matching (i) surface height $\eta$ and (ii) alongshelf transport $\psi_{x}$ along the common fluid boundary and by requiring $\psi=0$ along the solid wall at $y=0, L_{1}<x<L_{2}$ because it is a continuation of the coastal streamline. To match $\psi_{x}$ it is sufficient to match $\psi$ since $\psi$ is continuously differentiable in $x$ and $\psi_{-}=\psi_{+}$at the solid walls $(x=0, L)$.

For $L_{2}>L_{1}$ the matching conditions are

$$
\begin{gathered}
\psi_{+}=\left\{\begin{array}{cc}
\psi_{-}, & 0<x<L_{1} \\
0, & L_{1}<x<L_{2}
\end{array}\right. \\
\eta_{+}=\eta_{-}, \quad 0<x<L_{1}
\end{gathered}
$$

Integration of the alongshelf momentum equation (3.2) yields an expression for $\eta$

$$
\begin{aligned}
\eta_{-}= & \frac{1}{g h} \hat{A}\left(f \phi_{1 I}+\frac{\omega}{\hat{\ell}} \frac{d \phi_{1 I}}{d x}\right) e^{i(\hat{\ell} y-\omega t)} \\
& +\frac{1}{g h} \sum_{p=1}^{\infty} A_{p}\left(f \phi_{1 p}+\frac{\omega}{\ell_{1 p}} \frac{d \phi_{1 p}}{d x}\right) e^{i\left(\ell_{1 p} y-\omega t\right)} \\
\eta_{+}= & \frac{1}{g h} \sum_{q=1}^{\infty} B_{q}\left(f \phi_{2 q}+\frac{\omega}{\ell_{2 q}} \frac{d \phi_{2 q}}{d x}\right) e^{i\left(\ell_{2 q} y-\omega t\right)}+\frac{f}{g h_{0}} C e^{-i \omega t}
\end{aligned}
$$

where $C$ is a constant of integration corresponding to the Kelvin wave mode. This is easily seen by obtaining the solution for a divergent barotropic Kelvin wave and formally taking the linit of a rigid-lid boundary condition. Under a rigid lid the phase speed and horizontal spatial scales 
of the Kelvin mode become infinite thereby reducing the wave to a time varying but spatially uniform fluctuation in the pressure field throughout the channel. The velocities vanish as the reciprocal of phase speed and thus the Kelvin mode has no signal in the streamfunction. Despite this vanishing velocity, the degenerate Kelvin mode is still capable of propagating energy in a semi-infinite ocean because it no longer decays offshore. However, in the present analysis, the flow is confined to a coastal channel of finite width so the vanishing alongshelf velocity leads to vanishing energy flux. Thus a non-zero value of $C$, while indicating that a Kelvin wave is generated, is not associated with any propagation of energy. Equations (3.15), (3.16), (3.22) and (3.23) may be substituted into (3.20) and (3.21) to obtain

$$
\sum_{q=1}^{\infty} B_{q} \phi_{2 q}= \begin{cases}\hat{A} \phi_{1 I}+\sum_{p=1}^{\infty} A_{p} \phi_{1 p} & 0<x<L_{1} \\ 0, & L_{1}<x<L_{2}\end{cases}
$$

and

$$
\sum_{q=1}^{\infty} B_{q} \frac{\omega}{\ell_{2 q}} \frac{d \phi_{2 q}}{d x}+\frac{h}{h_{0}} f C=\hat{A} \frac{\omega}{\hat{\ell}} \frac{d \phi_{1 I}}{d x}+\sum_{p=1}^{\infty} A_{p} \frac{\omega}{\ell_{1 p}} \frac{d \phi_{1 p}}{d x}, \quad 0<x<L_{1}
$$

The orthogonality property (3.13) is applied as in Yeh (1975) in the evaluation of

$$
\begin{aligned}
& \int_{0}^{L_{2}}(3.24) e^{2 \lambda x} \phi_{2 n} d x \\
& \int_{0}^{L_{1}}(3.25) e^{2 \lambda x} \phi_{1 m} d x
\end{aligned}
$$

to obtain a set of linear equations for the $A_{p}$

$$
\begin{aligned}
E_{m} C & +\frac{1}{f L_{2}} \sum_{p=1}^{\infty}\left\{-\frac{\omega}{\ell_{1 p}} H_{m p}+\sum_{q=1}^{\infty} \frac{\omega}{\ell_{2 q}} J_{q p} K_{m q}\right\} A_{p} \\
& =\hat{A} \frac{1}{f L_{2}}\left(\frac{\omega}{\hat{\ell}} H_{m I}-\sum_{q=1}^{\infty} \frac{\omega}{\ell_{2 q}} J_{q I} K_{m q}\right) \quad m=1,2,3 \ldots
\end{aligned}
$$

where

$$
\begin{aligned}
E_{m} & =\frac{2}{L_{2}} \int_{0}^{L_{1}} \phi_{1 m} d x \\
H_{m p} & =2 \int_{0}^{L_{1}} \frac{d \phi_{1 p}}{d x} e^{2 \lambda x} \phi_{1 m} d x \\
J_{n p} & =\frac{2}{L_{2}} \int_{0}^{L_{1}} \phi_{1 p} e^{2 \lambda x} \phi_{2 n} d x \\
K_{m q} & =2 \int_{0}^{L_{1}} \frac{d \phi_{2 q}}{d x} e^{2 \lambda x} \phi_{1 m} d x
\end{aligned}
$$


After solving (3.28) for the $A_{p}$ and $C$, the $B_{\mathrm{q}}$ are obtained directly from

$$
B_{q}=\hat{A} J_{q I}+\sum_{p=1}^{\infty} J_{q p} A_{p}
$$

A parallel analysis for the case $L_{1}>L_{2}$ leads to a similar solution.

$$
\begin{gathered}
\frac{L_{1}}{L_{2}} E_{m}^{*} C+\frac{1}{f L_{2}} \sum_{q=1}^{\infty}\left\{\frac{\omega}{\ell_{2 q}} H_{m q}^{*}-\sum_{p=1}^{\infty} \frac{\omega}{\ell_{1 p}} K_{m p}^{*} J_{p q}^{*}\right\} B_{q} \\
=\hat{A} \frac{1}{f L_{2}} K_{m I}^{*}\left(\frac{\omega}{\hat{\ell}}-\frac{\omega}{\ell_{1 I}}\right) m=1,2,3 \ldots \\
A_{p}=-\hat{A} \delta_{I p}+\sum_{q=1}^{\infty} J_{p q}^{*} B_{q}
\end{gathered}
$$

where $E^{*}, H^{*}, J^{*}$ and $K^{*}$ are equivalent to the integrals $E, H, J$ and $K$ defined above but with $L_{1}$ replacing every occurrence of $L_{2}$ and vice versa.

To obtain a solution, the infinite set of equations (3.28) is truncated at $M+1$. Then the constant $C$ and the amplitudes $A_{p}$ are computed for the first $M$ modes. The results presented in the following section were obtained by selecting $M=40$ and by truncating the inner summation over $q$ at 60 modes. This was sufficient to determine the amplitudes of the lowest 5 modes to within 1 percent (and the lowest 15 modes to within 5 percent) of the solution obtained by using $M=100$ modes and an inner summation over $q$ of 200 modes.

To verify the accuracy of the solutions, a check on energy conservation was made. The alongshelf energy flux in the wave field is given by the time averaged product of pressure and alongshelf transport integrated across the shelf:

$$
\mathbf{F}=\int_{0}^{L} \overline{(g \eta) \cdot(h v)} d x
$$

The overbar denotes a time average. Huthnance (1975) showed that if a set of shelf waves have $\omega$ specified, the wave energy flux separates into contributions from individual modes with no contribution from interactions between modes. Evaluation of (3.36) for a single mode gives

$$
\mathbf{F}_{n}=\frac{1}{4} \frac{\left|A_{n}\right|^{2} f \lambda L}{h_{0}}\left(1-\frac{\omega \ell}{f \lambda}\right)
$$

where $A_{n}$ is the wave amplitude. The total radiated energy flux is obtained by summing this quantity over all propagating modes. (The evanescent modes have zero energy flux.) In all 
cases, the sum of the reflected and transmitted energy fluxes was within 0.01 percent of the incident energy flux.

It is also of interest to examine the form of the solution under the long-wave and accompanying low-frequency assumptions. While it might be expected that the abruptness of the coastline change would render the long-wave approximation invalid, a well behaved solution is obtainable for the case $L_{2}>L_{1}$. For long waves, the dispersion relation (3.12) simplifies to $\omega=2 \lambda f \ell_{n} /\left(k_{n}^{2}+\lambda^{2}\right)$ which admits only a single class of waves, all of which propagate and have group velocity in the same direction as phase speed. There are therefore no waves available to carry a reflected flux of energy so the coefficients $A_{p}$ in (3.15), (3.16), (3.22) and (3.23) vanish. Applying the matching conditions (3.20) and (3.21) and using the orthogonality relation as in (3.24) and (3.25) gives the simple solution

$$
\begin{gathered}
B_{q}=\hat{A} J_{q I} \quad q=1,2,3 \ldots \\
E_{m} C=\frac{1}{f L_{2}}\left(\hat{A} \hat{c} H_{m I}-\sum_{q=1}^{\infty} B_{q} c_{2 q} K_{m q}\right) \quad m=\text { any integer }
\end{gathered}
$$

where $\hat{c}$ and $c_{2 q}$ are the non-dispersive long-wave phase speeds $(\omega / \ell)$ of the incident and scattered waves, respectively.

For the case $L_{1}>L_{2}$, the boundary condition $\psi=0$ along $y=0, L_{2}<x<L_{1}$ cannot be satisfied and the long-wave limit fails.

\subsection{Results}

The features of the solution may be illustrated by investigating the effect of varying the five free parameters of the model. These are: $\omega / f$, the incident wave frequency relative to the inertial frequency; $I$, the incident wave mode number; and the three length scales $\lambda, L_{1}$ and $L_{2}$. The length scales form three dimensionless groups: $L_{2} / L_{1}, \lambda L_{1}$ and $\lambda L_{2} . L_{2} / L_{1}$ is the relative change in shelf width encountered by the wave while $\lambda L_{1}$ and $\lambda L_{2}$ represent the respective non-dimensional slope steepnesses. Inspection of (3.28) and (3.33) shows that the solution is unaffected by the deep ocean depth $h_{0}$. 
(a)

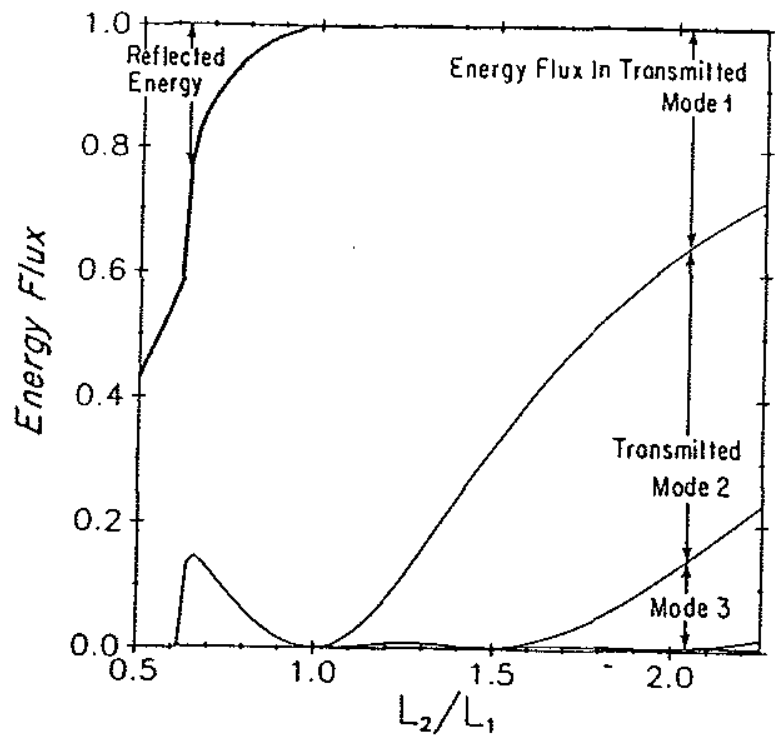

(b)

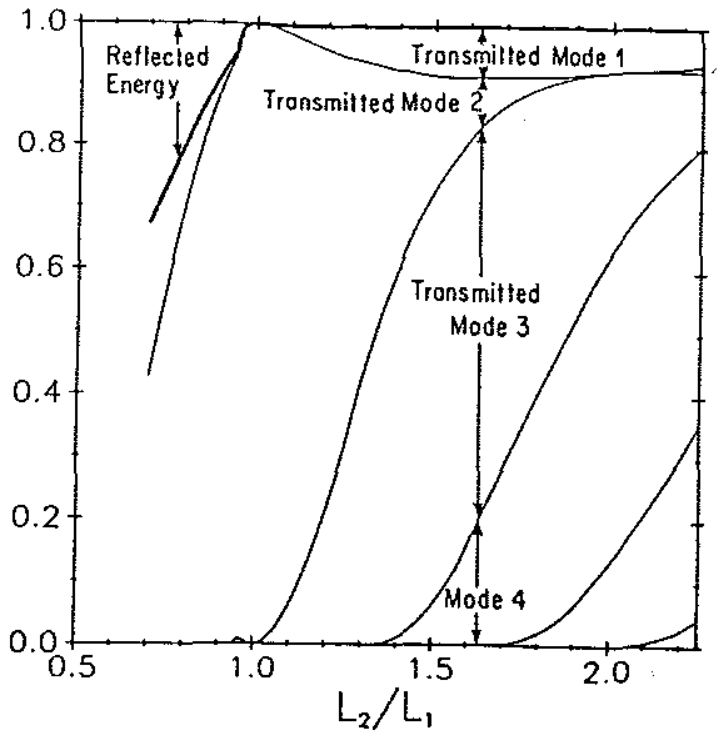

Figure 3-3: Modal energy fluxes normalized by the incident wave energy for $\omega / f=0.1, \lambda L_{1}=1.0$, and $L_{2} / L_{1}$ in the range 0.5 to 2.25 . The heavy line is the total transmitted energy. The light lines divide the energy between the various modes. (a) Mode 1 incident. (b) Mode 2 incident.

\section{(a) Relative shelf width $L_{2} / L_{1}$ varies}

The partitioning of the total scattered energy flux into individual transmitted and reflected modes is shown in Figure 3-3a for a mode 1 incident wave with $\omega / f=0.1$ and $L_{2} / L_{1}$ in the range 0.5 to 2.25 . The slope steepness over $y<0$ is fixed at $\lambda L_{1}=1$ so that the incident wave form does not vary. For $L_{2} / L_{1}<0.8$ an appreciable amount of energy (greater than 5 percent) is reflected. It was noted in Chapter 1 than realistic stratification is likely to appreciably alter, and may altogether eliminiate, short reflected Bsws. Therefore, the present barotropic solution is likely to be of limited applicability to realistic shelves over ranges of the model parameters for which the solution shows substantial reflection of energy. For this reason, discussion of narrowing shelf $\left(L_{2} / L_{1}<1\right)$ results will be henceforth omitted. For a widening shelf, energy is readily transferred into higher modes. For example, at $L_{2} / L_{1}=1.5$, transmitted mode 2 carries 33 percent of the scattered energy. Energy may also be transferred to modes lower than the incident. For example, Figure 3-3b shows the same situation as in Figure 3-3a but with a mode 2 incident wave. At $L_{2} / L_{1}=1.5,7$ percent of the incident energy has been transferred to transmitted mode 1 despite the cross-shelf structure being very different from that of the 
(a)

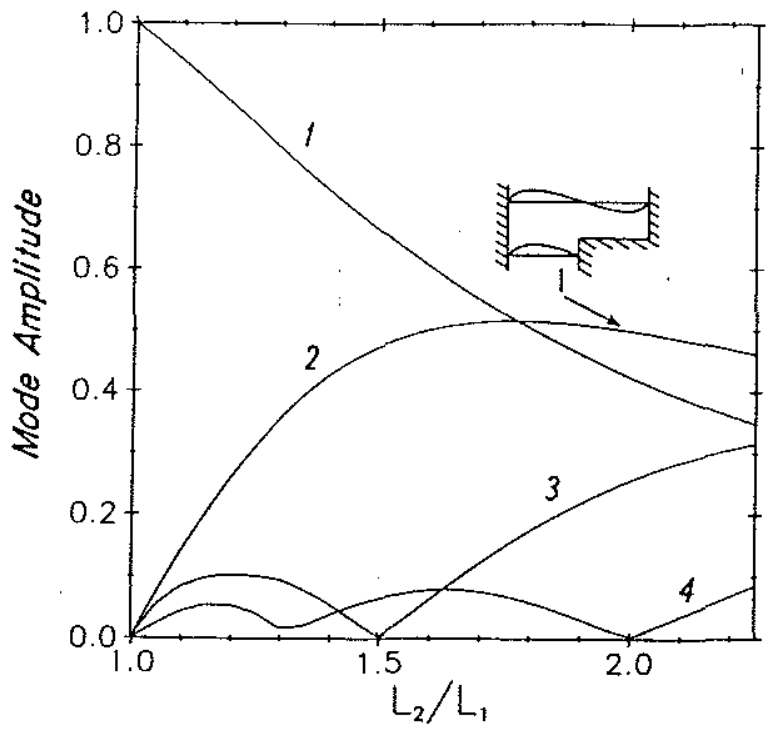

(b)

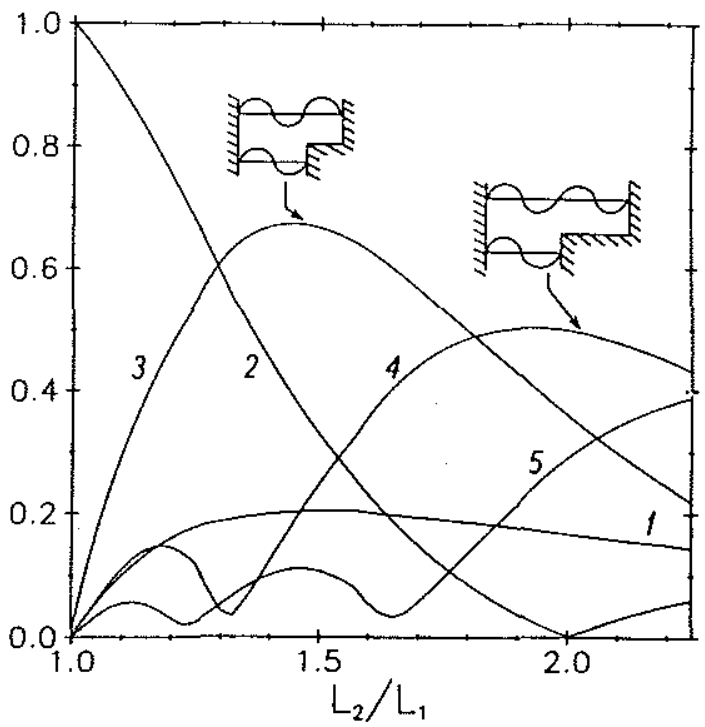

Figure 3-4: Magnitudes of transmitted mode amplitudes for the parameter range considered in Figure 3-3. Each mode $q$ attains its maximum amplitude near $L_{2} / L_{1}=q / I$ (where $I$ is the incident mode number) since this is where its cross-shelf structure most closely coincides with that of the incident wave.

incident wave. In general, the mode most readily excited is that which best fits the cross-shelf structure of the incident wave. A given transmitted mode $q$ has a cross-shelf structure of the form $\sin q \pi x / L_{2}$ which is similar to that of the incident wave, $\sin I \pi x / L_{1}$, in the vicinity of $q / L_{2}=I / L_{1}$. It may be expected then that each mode $q$ attains its maximum amplitude near $L_{2} / L_{1}=q / I$. This is verified in Figure 3-4 where the transmitted mode amplitudes for the cases in Figure 3-3 are plotted. At the local maxima, the amplitudes of the other modes do not vanish since a single transmitted mode is not sufficient to satisfy the condition $\psi=0$ along the solid wall at $y=0$. A significant contribution of the other modes is still required.

\section{(b) Frequency $\omega / f$ varies}

In this case the shelf geometry and incident mode number are fixed $\left(L_{2} / L_{1}=2.25 ; \lambda L_{1}=\right.$ $1.0 ; I=1$ ) while the wave frequency is varied. At low frequency, the modal partitioning of energy flux (Figure 3-5a) and the scattered mode amplitudes (Figure 3-5b) become independent of $\omega / f$. Their values are asymptotic to the results obtained from (3.38) and (3.39) indicating 
(a)

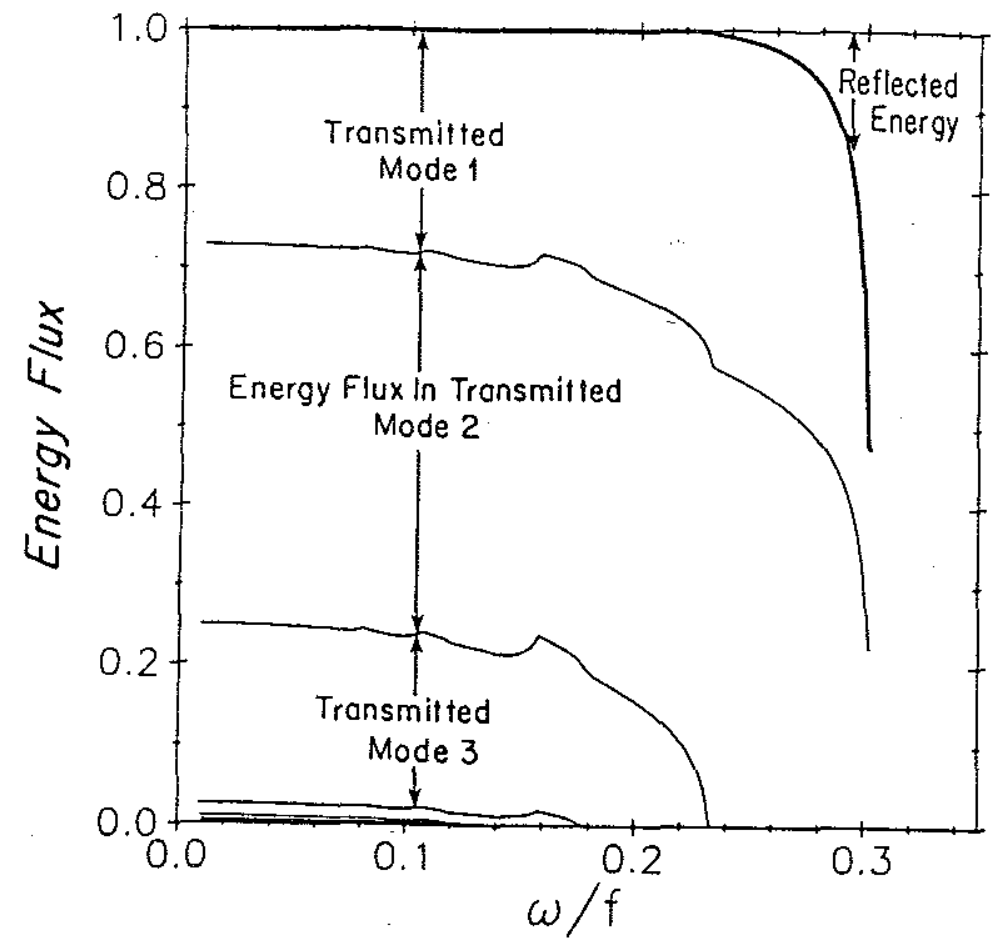

(b)

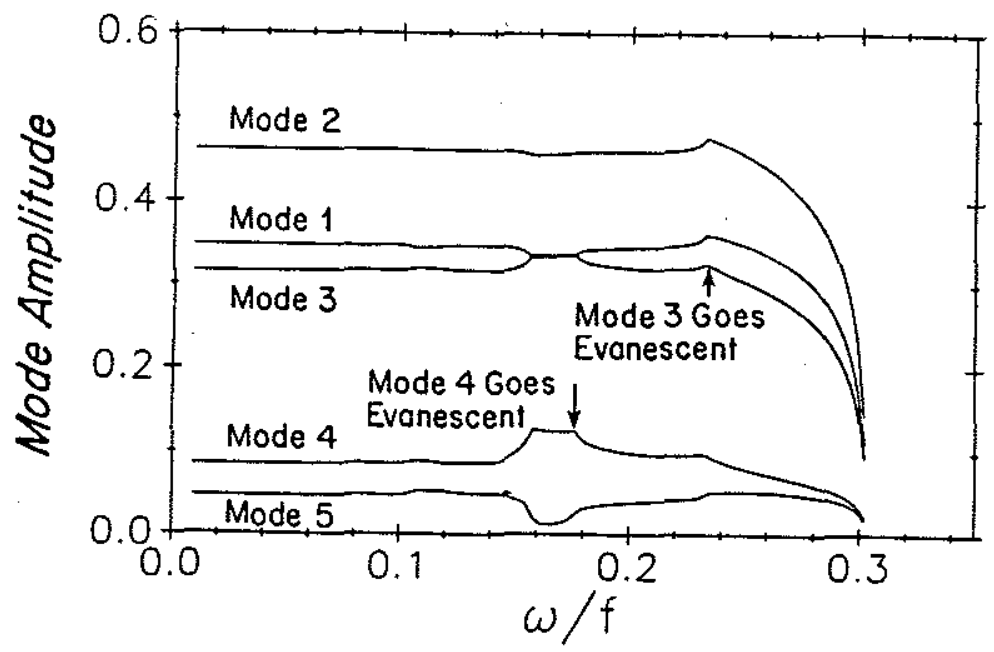

Figure 3-5: (a) Modal energy fluxes normalized by incident energy for mode 1 incident, $\lambda L_{1}=1.0$ and $L_{2} / L_{1}=2.25$ as a function of $\omega / f$. The heavy line is the total transmitted energy. Above the cutoff frequency for mode $3(\omega / f=0.232)$ reflection of energy occurs. (b) Corresponding magnitudes of mode amplitudes. As $\omega / f \rightarrow 0$ the scattered wave amplitudes become independent of frequency. 
that the long-wave limit appears valid despite the abruptness of the coastline variation.

As $\omega / f$ increases, the higher modes reach their cutoff frequencies and cease to propagate energy. In this example, for $\omega / f>0.232$ only two transmitted modes propagate. The energy carried by transmitted mode 3 diminishes rapidly as the cutoff frequency is approached and the group speed tends to zero. In passing through the cutoff frequency, the real part of the wavenumber does not change appreciably while the imaginary part increases slowly from zero. Thus the transition is not strongly manifested in the matching conditions, so the amplitudes of the remaining propagating transmitted modes do not alter significantly. This may be seen by examining the mode amplitudes plotted in Figure 3-5b. The energy flux carried in each of the remaining modes does not alter appreciably either, but the significant level of energy previously carried in mode 3 must be directed somewhere. It appears in the excitation of the lowest mode reflected wave. This shows that even for a widening shelf, the barotropic model predicts backscattering of shelf waves at high frequency. As noted above, realistic stratification is likely to eliminate the possibility of BSW reflection, and the application of the model results should be restricted to cases in which the higher transmitted propagating modes carry minimal amounts of energy; then reflected waves will not be generated as the cutoff frequency of those modes are approached.

\section{(c) Slope steepness $\lambda L$ varies}

The relative shelf width is held constant at $L_{2} / L_{1}=2.25$, a value which produced strong coupling between modes in the preceding results, and the slope steepnesses are varied. The energy flux partitioning is shown in Figure 3-6 for both mode 1 and mode 2 incident waves for $\omega / f=0.1$ as $\lambda L_{2}$ is varied from 1.125 to 4.0 . Correspondingly, $\lambda L_{1}$ varies from 0.5 to 1.78 . For small values of $\lambda L_{2}$, where the energy partitioning fluctuates, either the slope steepness or shelf width is small. On such a shelf, inspection of Figure 3-1 shows that few modes are available to propagate energy. In the mode 2 incident case it is seen that some reflection of energy occurs in the range $\lambda L_{2}<1.58$. As $\lambda L_{2}$ decreases through the value 1.58 , mode 5 ceases to propagate so that; by the same mechanism described above for increasing $\omega / f$, the significant energy flux previously carried by this mode is directed into the reflected modes. This suggests that the model results may not be applicable to narrow or weakly sloping shelves with realistic 

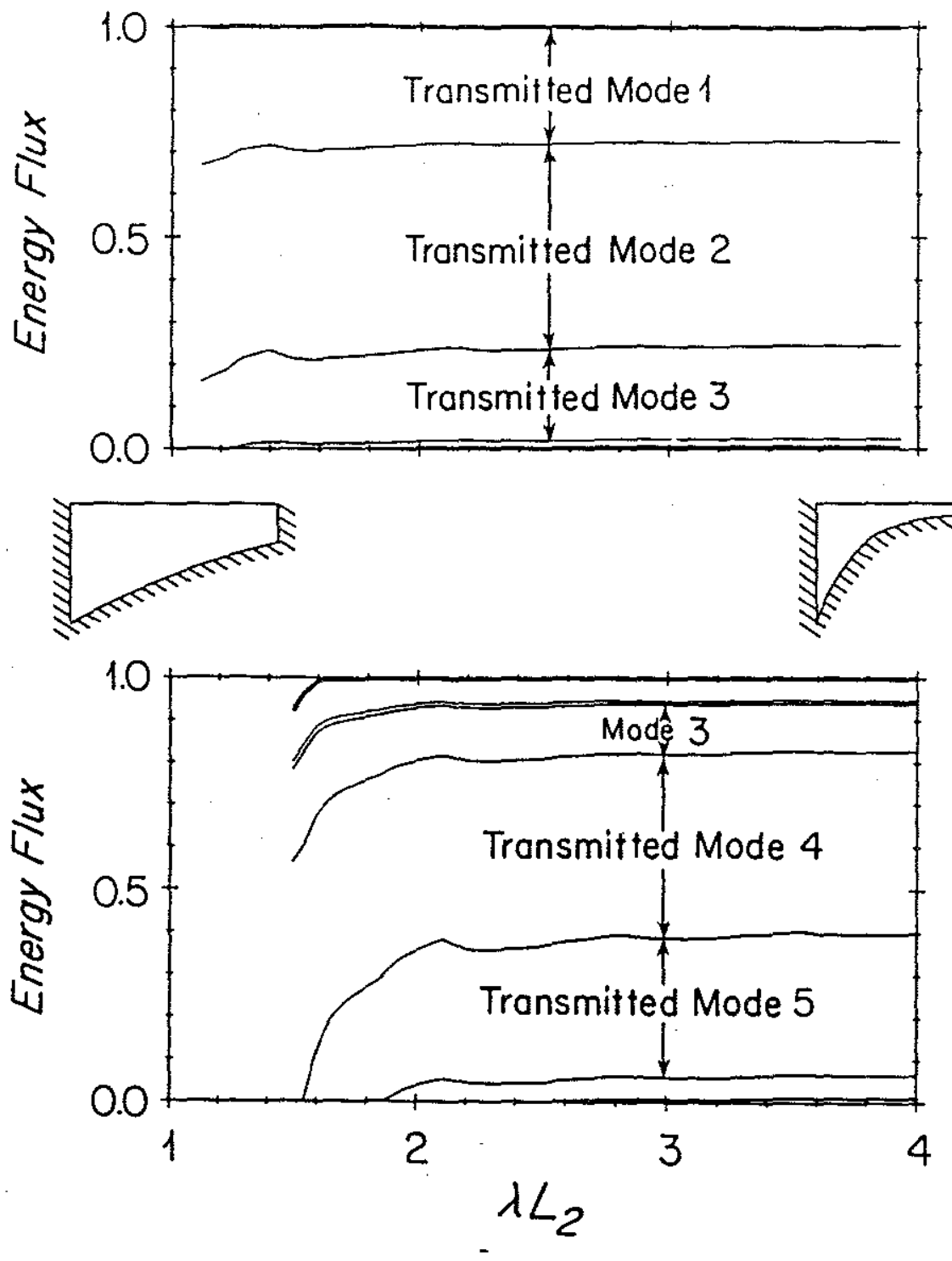

Figure 3-6: Normalized modal energy fluxes for $\omega / f=0.1, L_{2} / L_{1}=2.25$ as a function of $\lambda L_{2}$ (and $\lambda L_{1}$ ). Upper panel: Mode 1 incident. Lower panel: Mode 2 incident. The modal partitioning of energy becomes independent of $\lambda L$ at the moderate to high values typical of many continental shelves. 


\begin{tabular}{|c|c|c|c|c|c|c|c|c|c|c|c|}
\hline Mode number & 1 & 2 & 3 & 4 & 5 & 6 & 7 & 8 & 9 & 10 & 11 \\
\hline Amplitude & .346 & .461 & .316 & .085 & .047 & .043 & .013 & .026 & .005 & .013 & .005 \\
\hline \multirow[t]{2}{*}{ Alongshelf Scale } & \multicolumn{7}{|c|}{ (wavelength $/ L_{1}$ ) } & \multicolumn{4}{|c|}{$\left(e\right.$-folding length $\left./ L_{1}\right)$} \\
\hline & 42.3 & 14.0 & 6.45 & 3.56 & 2.16 & 1.36 & 0.77 & 0.20 & 0.13 & 0.10 & 0.09 \\
\hline
\end{tabular}

Table 3-1: Properites of first 11 transmitted modes for the case: mode 1 incident; $\omega / f=0.1 ; \lambda L_{1}=1.0$; $L_{2} / L_{1}=2.25$. Amplitudes are normalized by the incident mode amplitude. Characteristic alongshelf scale tabulated is the wavelength for the propagating modes and $e$-folding distance from $y=0$ for the evanescent modes. In this case the solution gives a value of $0.197 \hat{A}$ for the constant $C$.

stratification present. For moderate to larger values of $\lambda L_{2}$, the mode coupling is only weakly dependent on slope steepness which suggests that the results presented here are not particularly sensitive to details in the shape of the depth profile, and would therefore change little if a depth profile more general than the exponential were used.

\section{(d) Trapped field}

Over the entire parameter range considered in Figure 3-3 $\left(1<L_{2} / L_{1}<2.25 ; \lambda L_{1}=1.0\right.$; $\omega / f=0.1$ ) the amplitudes of the evanescent transmitted modes are an order of magnitude smaller than the principal propagating modes. The trapped field is therefore much weaker than the propagating field. As an example, transmitted wave amplitudes for the case $\omega / f=0.1$, $L_{2} / L_{1}=2.25, \lambda L_{1}=1.0$ are presented in Table 3-1. From these values are computed the current ellipses for the total field and the trapped field shown in Figure 3-7. The trapped field current ellipses are plotted with a scale 20 times greater than the total field ellipses which further indicates their lack of contribution to the overall flow. Also given in Table 3-1 are the alongshelf $e$-folding scales of the evanescent modes. Comparison with Figure 3-7b shows that the alongshelf extent of the evanescent field is approximately equal to the $e$-folding scale $\left(0.2 L_{1}\right)$ of the lowest trapped mode (mode 8). The total field shows the presence of a shadow zone of weak flow downstream $(y>0)$ of the coastline bend and no intensification near the corner. A 

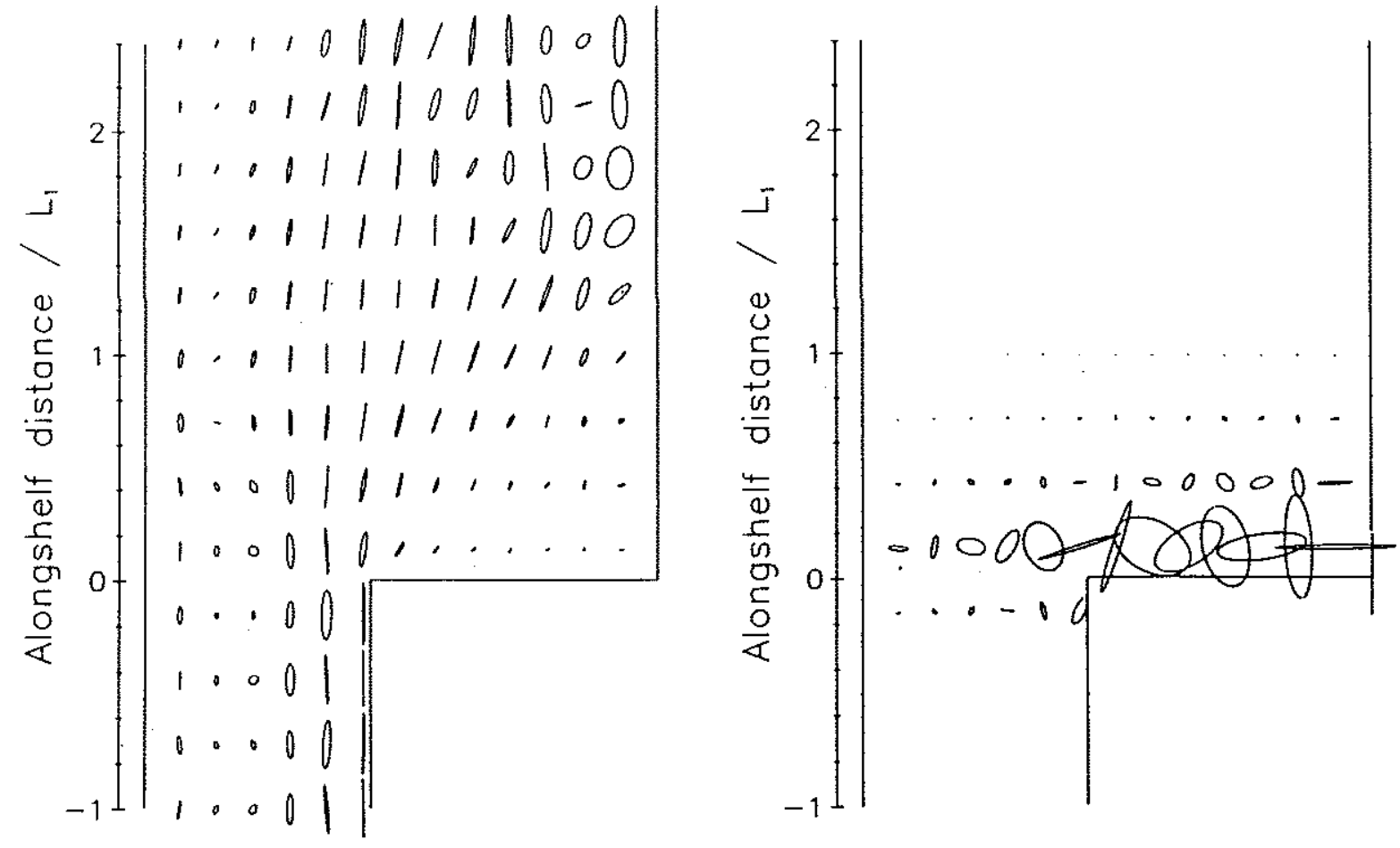

Figure 3-7: Current ellipses for $\omega / f=0.1, \lambda L_{1}=1, L_{2} / L_{1}=2.25$. Left: Total field. Right: Evanescent modes only. The velocity scale used to plot the evanescent modes is greater by a factor of 20 than that used for the total field which indicates the weakness of the trapped field relative to the propagating field.

plot of time and depth averaged kinetic energy (KE) density (Figure 3-8) shows that the KE density in the shadow zone is less than $1 / 20$ of that along the upstream coast $(y<0)$. Therefore both components of the velocity must be very weak in this region suggesting that the presence of a solid wall along, say, the unit KE density contour in Figure 3-8 should produce minimal alteration to the scattered wave solution since the boundary condition of no flow through the wall is already approximately satisfied.

The alongshelf extent of the shadow zone increases linearly with increasing $L_{2} / L_{1}$ and increases approximately as $(\omega / f)^{-1}$. This behavior is expected because of the tendency for low frequency flow to follow $f / h$ contours (isobaths on an $f$-plane). As $L_{2} / L_{1}$ increases, the relative change in coastal depth increases, so that the flow requires a longer alongshelf distance to make the adjustment, i.e. a longer shadow zone. Similarly, as the frequency decreases (for fixed $\left.L_{2} / L_{1}\right)$, the flow becomes more geostrophic and must follow the isobaths more closely, thus resulting in a longer shadow zone. The presence of the shadow zone suggests that shelf 


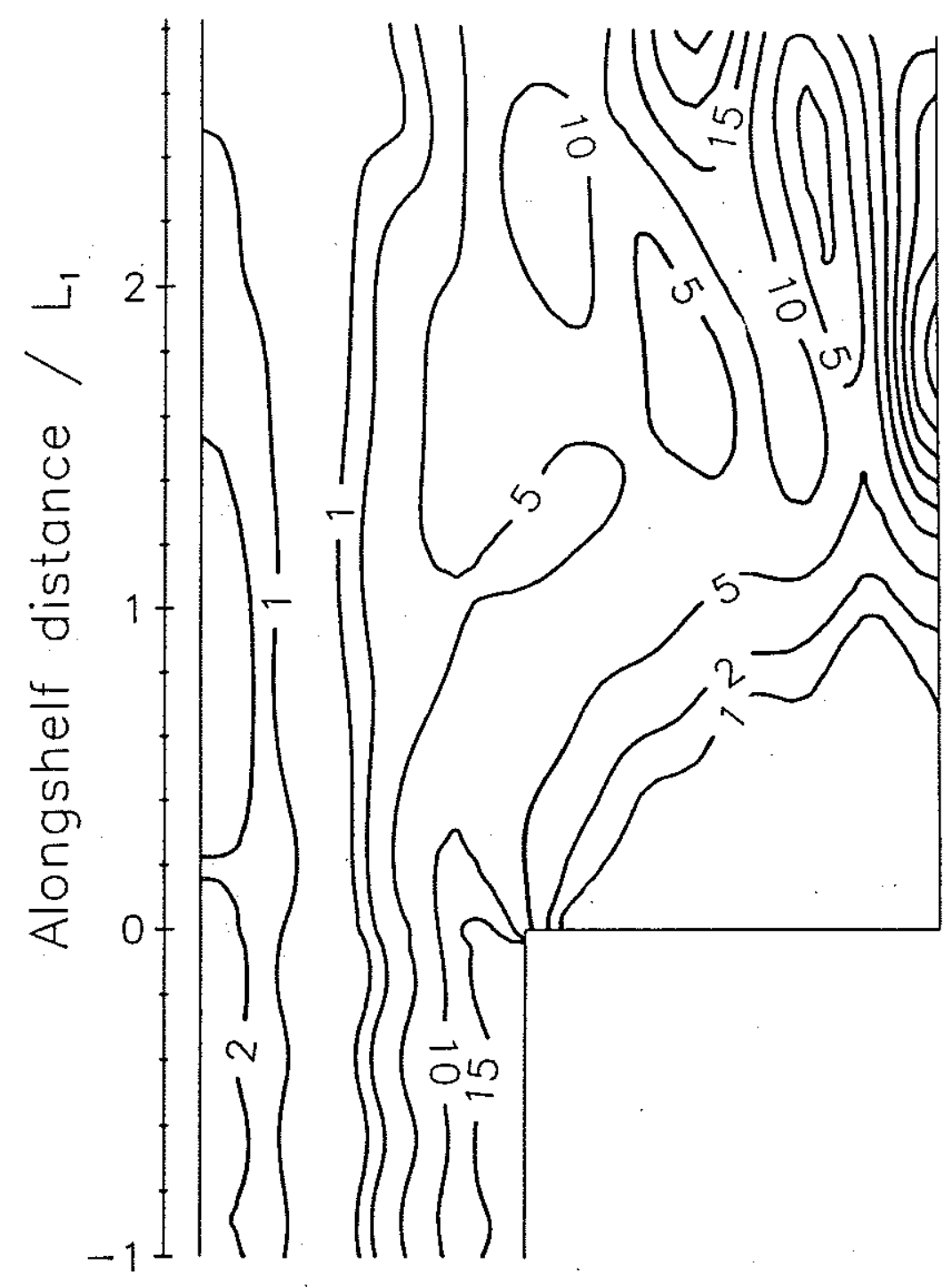

Figure 3-8: Time averaged kinetic energy (KE) density for $\omega / f=0.1, \lambda L_{1}=1, L_{2} / L_{1}=2.25$. A shadow zone of weak flow is present behind the coastline bend suggesting that were the, say, unit KE contour to be a solid wall, the solution should differ little. 
width variations which occur over an alongshelf distance comparable to or shorter than the length of the shadow zone should appear sufficiently abrupt to cause significant scattering.

\section{(e) Far field}

For the case considered in Table 3-1, the first three transmitted modes have comparable amplitudes but quite different wavelengths. These waves interact to produce a strong alongshelf modulation of the flow pattern downstream from the coastline bend. This can be seen in Figure 3-9 which presents an instantaneous contour plot of the surface height displacement. The simple cellular pattern of the crest and trough of the mode 1 incident wave scatters into a complex pattern with strong alongshelf and cross-shelf variability. The importance of the modal interaction is emphasized in Figure 3-10 which shows the phase and maximum range of surface height displacement along the coast. The uniform range and linear increase in phase upstream of the bend attest to the weakness of the backscattered field. Downstream, the varying range suggests that observers comparing sea-level oscillations at different locations along the coast could obtain erroneous estimates of barotropic shelf wave energy and phase speed if they were not cognizant of the scattering process in effect. Similarly, the cross-shelf structures of surface height variation (shown inset in Figure 3-10) exhibit local maxima in sea-level range well offshore, suggesting that weak shelf wave activity observed in coastal sea level could be misleading.

\subsection{Discussion}

Before considering the implications of the preceding results for shelf wave scattering in a real coastal ocean, it is instructive to review the limitations of the model from which the results were derived.

Care has been taken in the present analysis to avoid solutions where energy is scattered into barotropic short waves which would be substantially altered and might be altogether absent in a realistically stratified ocean. In general, reflected short waves are not generated when the following conditions apply: (i) The shelf widens $\left(L_{2} / L_{1}>1\right)$, (ii) the slope steepness parameters are not too small $\left(\lambda L_{2}\right.$ greater than approximately 2$)$ and (iii) wave frequencies are small $(\omega / f$ 


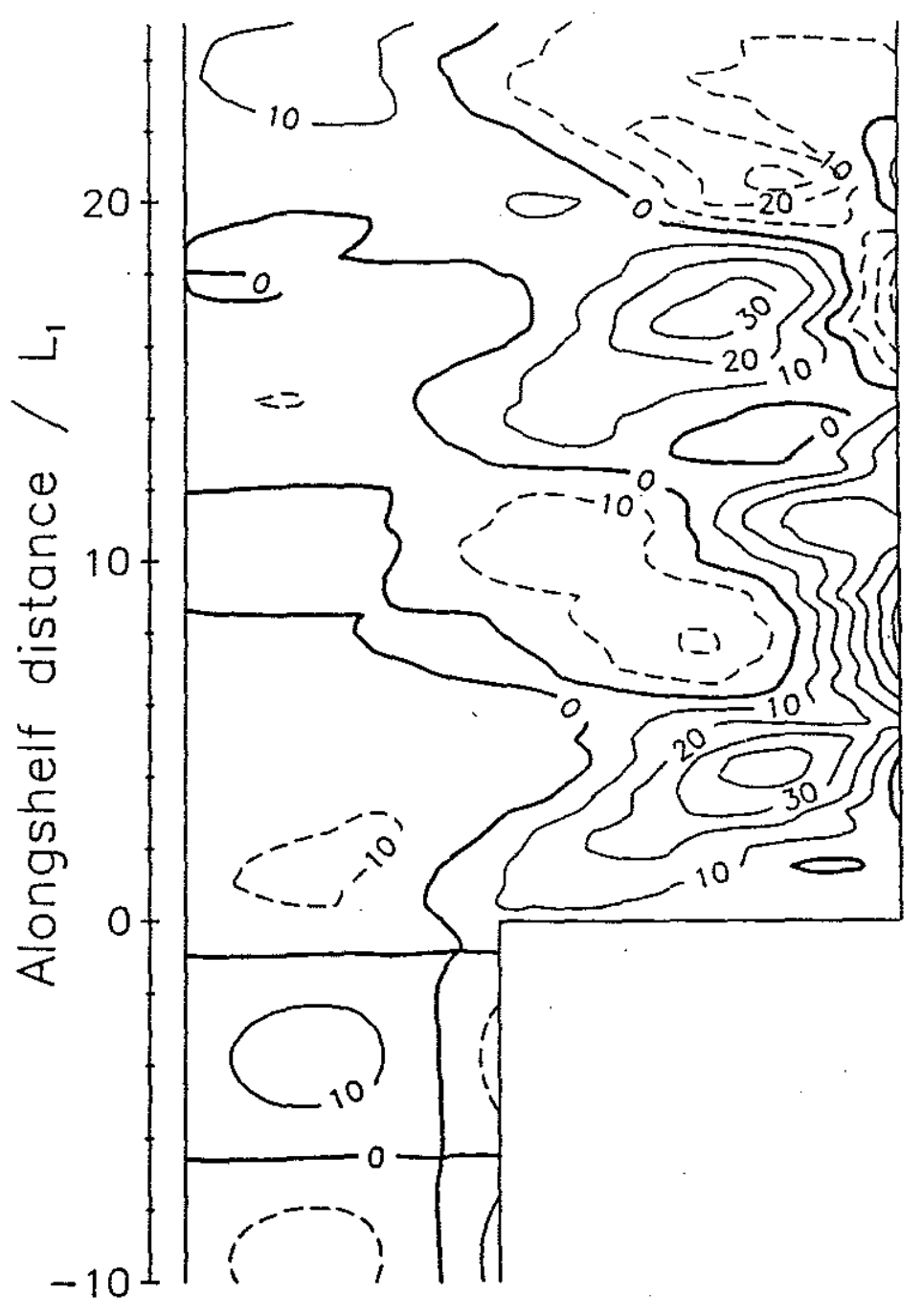

Figure 3-9: Instantaneous sea level displacement for $\omega / f=0.1, \lambda L_{1}=1, L_{2} / L_{1}=2.25$. The simple cellular pattern of a mode 1 incident wave is scattered into a complex pattern of interacting modes. The alongshelf scale is compressed. 

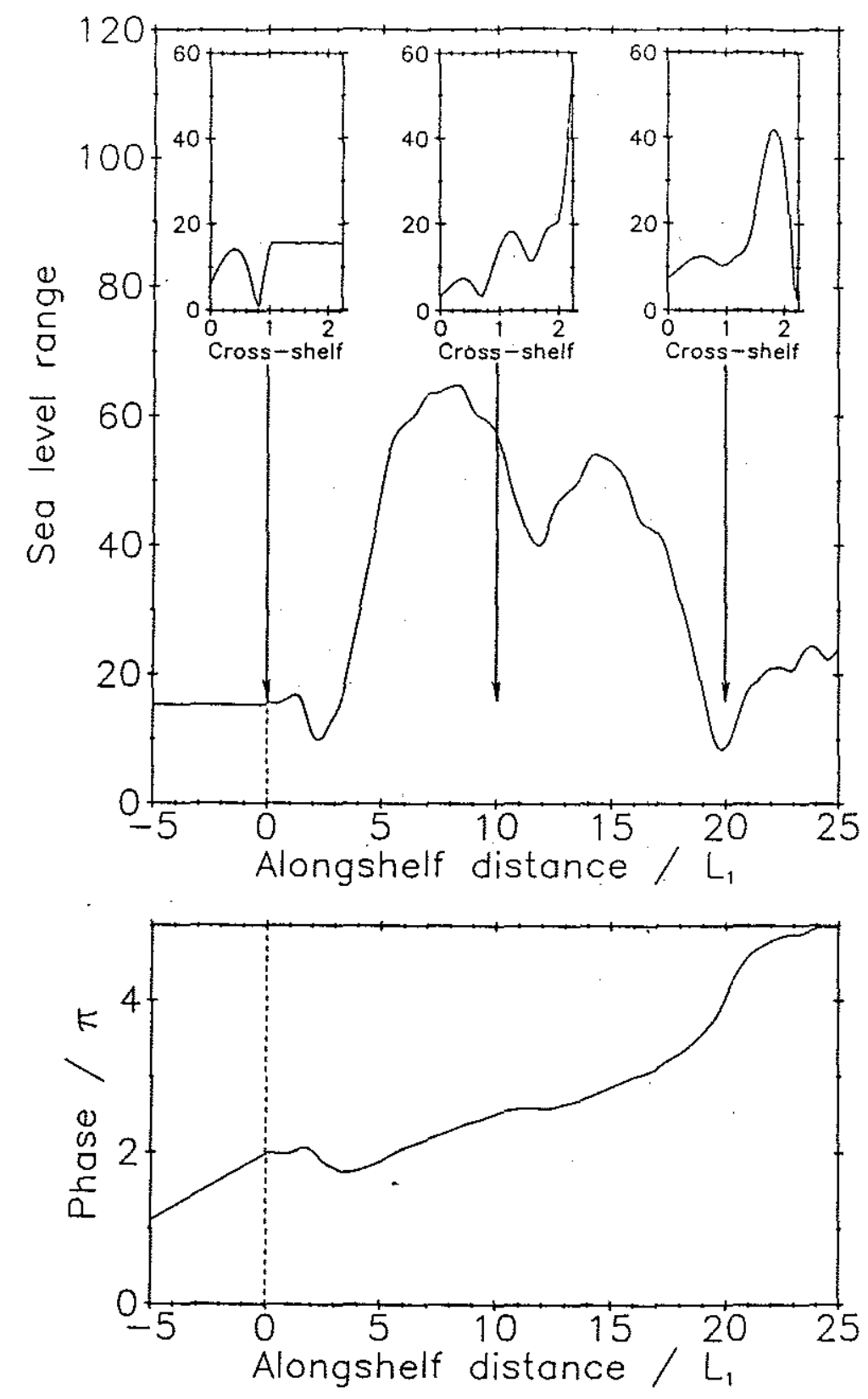

Figure 3-10: Range and phase of sea-level fluctuations along the coast (i.e. $\left.x=L_{1}, y<0 ; x=L_{2}, y\right\rangle 0$ ) for $\omega / f=0.1, \lambda L_{1}=1, L_{2} / L_{1}=2.25$. The cross-shelf structure at selected stations (including the coast along $y=0, L_{1}<x<L_{2}$ ) is shown inset. 
less than approximately 0.2 ).

Together, these conditions effectively ensure that there are a large number of propagating modes available to transmit energy forward of the coastline bend, so that energy may be conserved without exciting reflected waves. For a given shelf geometry, stratification increases the frequency at which a wave propagates, so there will be more propagating modes in the stratified case than in the barotropic case. This suggests that the results of the present analysis may be applicable qualitatively over a broader range of parameters than that defined above.

Neither the exponential topography nor the abruptness of the coastline bend appear to be limiting assumptions. The scattered wave amplitudes are not strongly dependent on $\lambda L$ for values typical of many continental shelves. This indicates that details of the shelf profile do not significantly affect the scattering process. The presence of a shadow zone of very weak currents behind the coastline bend suggests that a more general, less abrupt variation in shelf width, occurring over an alongshelf distance shorter than or comparable to the length of the shadow zone, should still produce the strong mode coupling evident in the present solution.

The solution to the model equations is assumed to be periodic both in space and time. However, the scattering of a non-dispersive shelf wave 'pulse' of limited alongshelf extent can be considered by superposing many periodic solutions. At the low-frequency limit, the present solution shows that a continuous wave is scattered into several modes with the partitioning being nearly independent of frequency and wavenumber. Under these conditions, the wavenumber spectra $^{1}$ of the scattered wave components will be proportional to the wavenumber spectrum of the incident wave (see Appendix A) so that the components of each scattered mode will combine to produce a wave pulse of that mode. Thus, a long shelf wave pulse will scatter into a train of pulses of differing modes, each travelling at its own free-wave speed and having an amplitude given by the periodic wave solution obtained here. This behavior is exact if the long-wave limit is assumed a priori.

So far, bottom frictional damping has not been included in the model. One might expect that frictional effects would eliminate the need to identify high mode waves in shelf wave observations because the higher mode waves would be damped over a length scale much shorter

\footnotetext{
${ }^{1}$ The term spectrum refers to the complex Fourier transform into wavenumber space of the alongshelf structure of the shelf wave pulse and should not be confused with a power spectrum which contains no phase information.
} 
than that of the lowest mode. To demonstrate that this is not the case, the analysis of Brink and Allen (1978) can be applied to the present model. The effect of weak bottom friction at lowest order is to damp barotropic free waves exponentially over a length scale $\Delta_{q}=\left(E^{1 / 2} a_{q q}\right)^{-1}$. Here, $E$ is an Ekman number defined by

$$
E^{1 / 2}=\left(\frac{1}{2} A_{v} / f\right)^{1 / 2} / h_{0}
$$

where $A_{v}$ is a constant vertical eddy viscosity and

$$
a_{q q}=\frac{1}{\lambda L} \int_{0}^{L} \frac{h_{0}^{2}}{h^{2}} \frac{d \phi_{q}}{d x} \frac{d \phi_{q}}{d x} d x
$$

Using this result, the effect of friction may be introduced into the present solution by exponentially damping each transmitted mode from its initial value at $y=0$. Frictional coupling between modes enters at second order. The above definitions differ slightly from Brink and Allen's because, in the present notation, $h_{0}$ is the depth at the offshore boundary rather than the depth at the coast. Consequently, the Ekman number used by Brink and Allen $(1978,1983)$ in their calculations $\left(E_{0}^{1 / 2}=0.06\right)$ must be multiplied by the relative depth change across the shelf to get $E^{1 / 2}$. For the example parameters used in Table 3-1 this gives $E^{1 / 2}=6.7 \times 10^{-4}$ and the corresponding values of $\Delta_{q}$ for the first three modes are: $\Delta_{1}=14.7 L_{2}, \Delta_{2}=5.96 L_{2}$ and $\Delta_{3}=3.16 L_{2}$. With friction included, the alongshelf modulation of coastal surface height range (Figure 3-11) due to interacting modes is less pronounced than in the frictionless case (Figure 3-10), but it may still be important observationally.

Periodic wind forcing along a coast is expected to radiate free waves of all modes at the forcing frequency (Gill and Schumann, 1974). The lowest modes are generally the most readily excited suggesting that the energy propagating in mode 1 should dominate that of higher modes. Scattering can act to reverse this situation. For example, consider two waves of the same frequency simultaneously incident upon a coastline discontinuity, such as might be generated by distant wind forcing. Table 3-2 shows two different superpositions of the scattered waves resulting from an incident second mode wave having half the amplitude of the incident first mode wave. The magnitude of mode 3 relative to mode 1 changes markedly depending on whether the incident waves are in phase $\left(\hat{A}_{2}=1 / 2\right)$ or $180^{\circ}$ out of phase $\left(\hat{A}_{2}=-1 / 2\right)$ at $y=0$. Such a shift in relative phase of the incident waves could occur if the generating weather system were to be displaced up or down the coast. This simple idea, in which the apparent scattering 

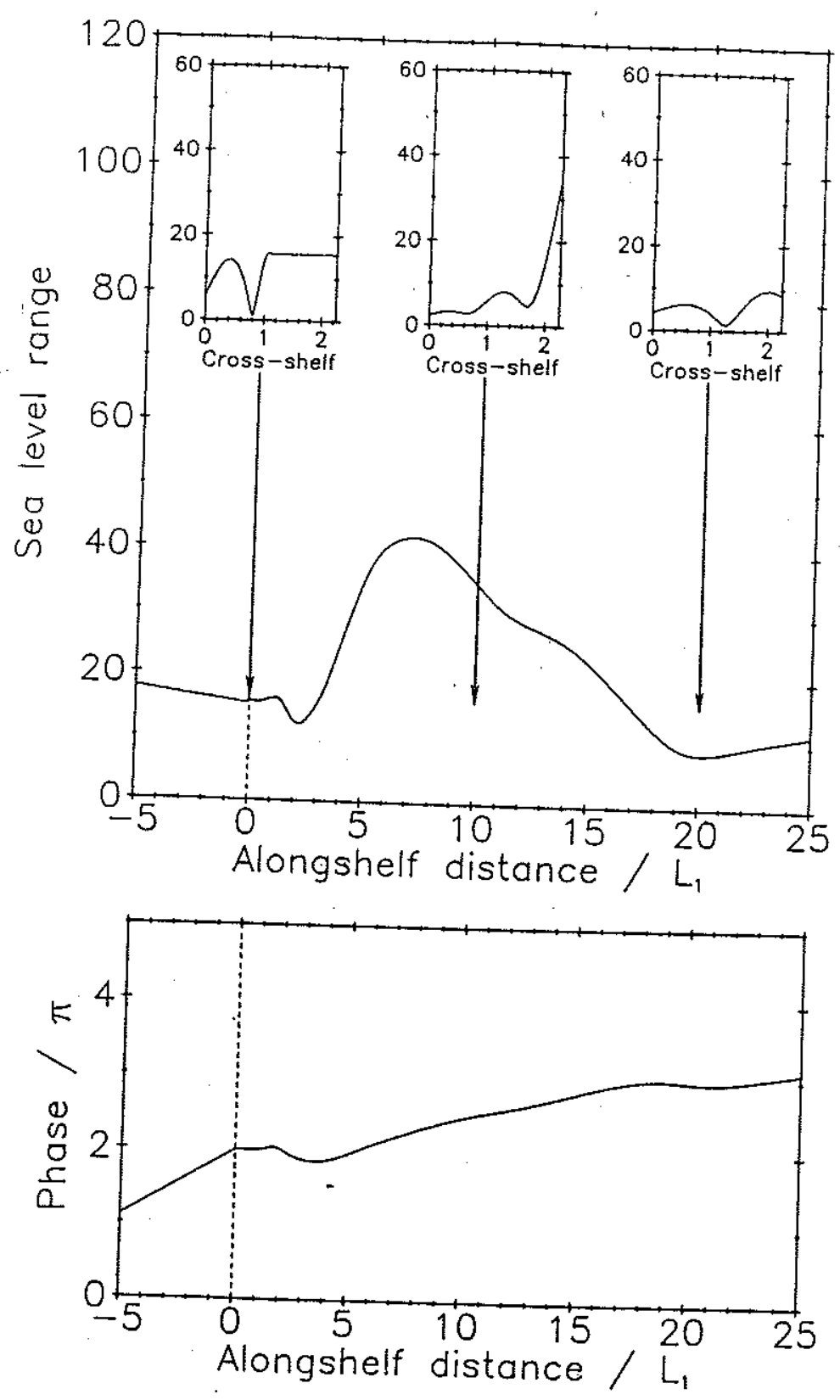

Figure 3-11: Same as Figure 3-10 but with weak bottom friction included. 


\begin{tabular}{|c|c|c|c|c|c|c|}
\hline \multirow[b]{2}{*}{$\begin{array}{c}\text { Mode } \\
\quad q \\
\end{array}$} & \multicolumn{2}{|c|}{$\begin{array}{c}\text { Amplitudes of } \\
\text { Transmitted Modes* }\end{array}$} & \multicolumn{2}{|c|}{$\begin{array}{c}\text { Incident waves in phase } \\
\text { at } y=0\end{array}$} & \multicolumn{2}{|c|}{$\begin{array}{c}\text { Incident waves } 180^{\circ} \text { out } \\
\text { of phase at } y=0\end{array}$} \\
\hline & $B_{1 q}$ & $B_{2 q}$ & $\left|B_{1 q}+\frac{1}{2} B_{2 q}\right|$ & Energy & $\left|B_{1 q}-\frac{1}{2} B_{2 q}\right|$ & Energy \\
\hline 1 & $0.346+i 0.001$ & $-0.145-i 0.003$ & 0.274 & 0.146 & 0.419 & 0.342 \\
\hline 2 & $0.461+i 0.001$ & $-0.059-i 0.002$ & 0.431 & 0.351 & 0.490 & 0.455 \\
\hline 3 & $0.316-i 0.001$ & $0.219+i 0.002$ & 0.425 & 0.323 & 0.206 & 0.076 \\
\hline
\end{tabular}

Table 3-2: Superposition of scattered modes from two incident waves of frequency $\omega / f=0.1$. The geometry is $\lambda L_{1}=1.0, \lambda L_{2}=2.25, L_{2} / L_{1}=2.25$. Energy fluxes are normalized by the incident energy flux.

* First subscript denotes incident mode number.

ability of a particular shelf geometry depends on the incident wave pattern, is suggestive of the confounding role that scattering can play in the interpretation of shelf wave observations.

Some qualitative features of the present solution have apparently been observed recently by Griffin and Middleton (1986) off the east coast of Australia at the southern end of the Great Barrier Reef. There, the promontory of Fraser Island causes a sudden increase in shelf width to the north. Over the wider shelf, Griffin and Middleton fit observed alongshelf velocities to a sum of free CTW modes. Near the coast the modes added in near antiphase to produce currents much weaker than those observed farther offshore. Closer to Fraser Island they added even closer to antiphase which is suggestive of a CTW shadow zone behind Fraser Island. The occurrence of a shadow zone and the indication that significant levels of energy are present in multiple modes are both consistent with scattering due to the changing shelf width. Furthermore, Griffin and Middleton conclude that the current variability north of Fraser Island results from CTws which propagate into the study region from somewhere south of Fraser Island. The observations made by Griffin and Middleton (1986) are discussed further in Chapter 6. 


\subsection{Summary}

A barotropic shelf wave of frequency $\omega$ travelling along a shelf whose width changes abruptly from $L_{1}$ to $L_{2}$ at some point scatters into all allowable modes of that frequency. A solution for the scattered wave amplitudes has been obtained for the case of an exponential cross-shelf depth profile, $h(x)=h_{0} e^{-2 \lambda x}$. Discussion is limited to solutions in which reflected short waves, which might not be present in a realistically stratified ocean, are not generated. This requires that the shelf widens $\left(L_{2} / L_{1}>1\right)$, the slope steepness is not too small $(\lambda L>$ roughly 2$)$, and the wave frequency is low $(\omega / f<$ roughly 0.2$)$. Within this parameter range, the following barotropic results should remain valid for a stratified coastal ocean.

1. The solution is relatively insensitive to the steepness of the exponential profile $(\lambda L)$ which suggests that the results are qualitatively applicable to more general cross-shelf depth profiles.

2. The transmitted mode which is most readily excited is that which has a cross-shelf structure most closely coinciding with that of the incident wave. For a widening shelf, energy is therefore readily transferred to higher modes.

3. For a widening shelf, there is a shadow zone of weak flow behind the coastline bend. The strong mode coupling is therefore primarily a result of the absolute width change and not merely its abruptness.

4. A non-dispersive shelf wave 'pulse' of limited alongshelf extent scatters into a train of similarly shaped waves of all allowable modes, each propagating at its own free-wave speed.

5. The interaction of the scattered modes strongly modulates the magnitude and phase progression of the flow field downstream of the scattering region. This modulation occurs over an alongshelf distance too short for the effect to be eliminated by weak frictional damping of the higher modes.

6. The scattered waves resulting from multiple incident waves of different modes can interfere to enhance the energy in some modes while reducing that in others. 
The above analytical study represents an initial step toward understanding the importance of coastal-trapped wave scattering in coastal processes. However, the neglect of stratification has placed a severe limitation on the usefulness of the results by excluding narrowing shelves from discussion. The next chapter describes a numerical mor' ' used to investigate CTW scattering by more general topographic variations in a realistically stratified coastal ocean. The results of the numerical study are compared with the above analytical results in the succeeding chapter. 


\section{Chapter 4}

\section{The Numerical Model}

\subsection{Introduction}

The numerical experiments to be discussed in Chapter 5 were conducted using a customized version of a four-dimensional $(x, y, z, t)$ primitive equation ocean circulation model developed by Dr. Dale Haidvogel of The Johns Hopkins University. The Haidvogel primitive equation model ( $P M$ ) is ideally suited to studies of CTW scattering because it allows for the specification of:

- irregular coastal geometry through the use of boundary-fitted orthogonal curvilinear coordinates in the horizontal

- irregular bottom topography by employing a stretched 'sigma' coordinate in the vertical

- arbitrary density stratification

- arbitrary open boundary conditions

In the numerical experiments the PEM is configured as a 'numerical wave-tank' in which a free $\mathrm{CTW}$, introduced at the upstream (in the sense of CTW propagation) open boundary of the computational domain, propagates through a region of irregular coastal geometry and/or bottom topography where it scatters into several CTWs of differing mode number. The scattered waves subsequently propagate out the downstream open boundary of the domain. 


\subsection{The Haidvogel Primitive Equation Model}

The formulation, implementation and testing of the PEM have been described in detail by Haidvogel et al. (1988) so only a brief synopsis of the model is presented here.

The primitive equations are derived from the incompressible Navier-Stokes equations of fluid flow by making the Boussinesq and hydrostatic approximations (e.g. Cox, 1985). In the Boussinesq approximation density variations are neglected in the momentum equations except in their contribution to the gravitational (or buoyancy) body force in the vertical momentum equation. Under the hydrostatic approximation it is assumed that the vertical pressure gradient balances the gravitational force. The primitive equations may be written

$$
\begin{aligned}
\mathbf{u}_{t}+(\mathbf{u} \cdot \nabla) \mathbf{u}+w \mathbf{u}_{z}+f \mathbf{k} \times \mathbf{u} & =-\nabla \phi+\left(\nu \mathbf{u}_{z}\right)_{z}+\mathbf{F}^{\mathbf{u}} \\
\phi_{z} & =-\frac{\rho g}{\rho_{0}} \\
\rho_{t}+(\mathbf{u} \cdot \nabla) \rho+w \rho_{z} & =\left(\kappa \rho_{z}\right)_{z}+\mathbf{F}^{\rho} \\
\nabla \cdot \mathbf{u}+w_{z} & =0
\end{aligned}
$$

where the notation used is the same as that in Chapter 3 except that $\phi=p / \rho_{0}$ where $p$ is the perturbation pressure. The horizontal velocity vector $\mathbf{u}$ has components $(u, v), \mathbf{k}$ is the unit vector along the vertical $z$ axis, $w$ is the vertical velocity, $\nabla$ is the horizontal gradient operator, $\nu$ and $\kappa$ are vertical diffusion coefficients for momentum and density, and the density is

$$
\rho_{\text {total }}=\rho_{0}+\rho(x, y, z, t)
$$

It is assumed that $\rho_{0} \gg \rho$.

The terms $F^{\mathbf{u}}$ and $F^{\rho}$ represent small-scale horizontal mixing processes unresolved by the grid scale of the numerical model. In practice, they are parameterized as simple diffusion terms and are retained to suppress small-scale computational noise. The numerical formulation of the PEM allows the prescription of any dynamically consistent surface, bottom and lateral boundary conditions provided the surface is assumed to be a rigid lid. The rigid lid eliminates surface gravity waves which would otherwise severely constrain the size of the numerical time step. The boundary conditions chosen for the present study are outlined in section 4.5 .

To accomodate irregular lateral boundaries, equations (4.1) through (4.4) are solved in orthogonal horizontal coordinates fitted to the coastal and offshore boundaries of the numerical 
domain. The generation of the boundary-fitted coordinate systems is described in section 4.3 . To accomodate irregular bottom topography a stretched vertical 'sigma' coordinate is introduced which effectively flattens out the variable bottom at $z=-h(x, y)$. The new vertical coordinate is defined by $\sigma=1+2 z / h$ and therefore spans the rangn $-1<\sigma<1$. This geometric simplification is achieved at the expense of complicating the governing equations (see Haidvogel et al., 1988).

The governing equations of the PEM are discretized using traditional centered second-order finite-differences in the horizontal and a spectral method in the vertical. The spectral technique represents the vertical dependence as an expansion in a set of Chebyshev polynomials. The solution of the discrete equations proceeds in a sequence of steps. First, the time rate of change of the depth-averaged vorticity is calculated by taking the depth-averaged curl of the momentum equations. Then, by application of the leapfrog-trapezoidal time-differencing scheme, an updated value of the vorticity is obtained from which the streamfunction is calculated through the solution of a boundary-value elliptic equation. The depth-averaged components of the velocity field are obtained directly from the streamfunction. The internal velocity field (that part of the velocity which has zero depth average) is obtained by time stepping the momentum equations. The separation of internal and depth-averaged components is necessary because the right-handside of the momentum equations contain an unknown contribution to the pressure field (which is uniform with depth) arising due to the rigid lid. The density equation is advanced in time by a leapfrog-trapezoidal time step. Once the $u, v$ and $\rho$ fields have been obtained at the new time level, the baroclinic pressure field is evaluated by integrating the hydrostatic relation (4.2), and the vertical velocity by integrating the continuity equation (4.4).

In order to study freely propagating linear inviscid CTWs it is appropriate to simplify the primitive equations further by omitting the non-linear terms and vertical diffusion terms from the numerical calculations. The magnitude of the omitted non-linear terms is examined at the end of section 5.4. Strictly speaking, the horizontal mixing terms should also be omitted, but as is frequently found in primitive equation models, some horizontal friction must be retained in the momentum equations to control small-scale computational noise. 


\subsection{Orthogonal Curvilinear Coordinates}

The computational domain of the PEM is a rectangular Arakawa C grid (e.g. Haidvogel et al., 1988) with uniform spacing in the orthogonal horizontal curvilinear coordinates. The mapping of an irregular physical domain to the regular computational coordinates introduces metric coefficients into the differential operators of the governing equations. While for some simple geometries, e.g. polar coordinates, these metric coefficients can be calculated analytically, in general a coordinate mapping which conforms to the irregular boundaries of a region of geophysical interest must be computed numerically.

The coordinate transformation sought is of the form $x(\xi, \eta), y(\xi, \eta)$ such that the computational coordinates $\xi, \eta$ are orthogonal and the boundaries of the physical domain, described in Cartesian $x, y$ coordinates lie along lines of constant $\xi$ or $\eta$. Once the coordinate mapping is known, the metric coefficients of the transformation can be readily computed. The metric coefficients $m$ and $n$ of the orthogonal curvilinear coordinate system relate differential distances in the $\xi, \eta$ directions to actual physical arc lengths

$$
(d s)_{\xi}=\frac{d \xi}{m} \text { and }(d s)_{\eta}=\frac{d \eta}{n}
$$

A line element of length $d s$ therefore satisfies

$$
d s^{2}=\frac{d \xi^{2}}{m^{2}}+\frac{d \eta^{2}}{n^{2}}
$$

In terms of Cartesian coordinates $x, y$ the line element may also be written

$$
\begin{aligned}
d s^{2} & =d x^{2}+d y^{2} \\
& =\left(x_{\xi} d \xi+x_{\eta} d \eta\right)^{2}+\left(y_{\xi} d \xi+y_{\eta} d \eta\right)^{2} \\
& =\left(x_{\xi}^{2}+y_{\xi}^{2}\right) d \xi^{2}+\left(x_{\eta}^{2}+y_{\eta}^{2}\right) d \eta^{2}+2\left(x_{\xi} x_{\eta}+y_{\xi} y_{\eta}\right) d \xi d \eta
\end{aligned}
$$

where subscripts denote partial differentiation. In an orthogonal coordinate system, $x_{\xi} x_{\eta}+$ $y_{\xi} y_{\eta}=0$ (e.g. Margenau and Murphy, 1943, Chapter 5), so that the last term in (4.8) vanishes and comparison with (4.7) shows how $m$ and $n$ can be computed from Cartesian coordinates defined as functions of $\xi$ and $\eta$ :

$$
\begin{aligned}
& m=\left(x_{\xi}^{2}+y_{\xi}^{2}\right)^{-\frac{1}{2}} \\
& n=\left(x_{\eta}^{2}+y_{\eta}^{2}\right)^{-\frac{1}{2}}
\end{aligned}
$$




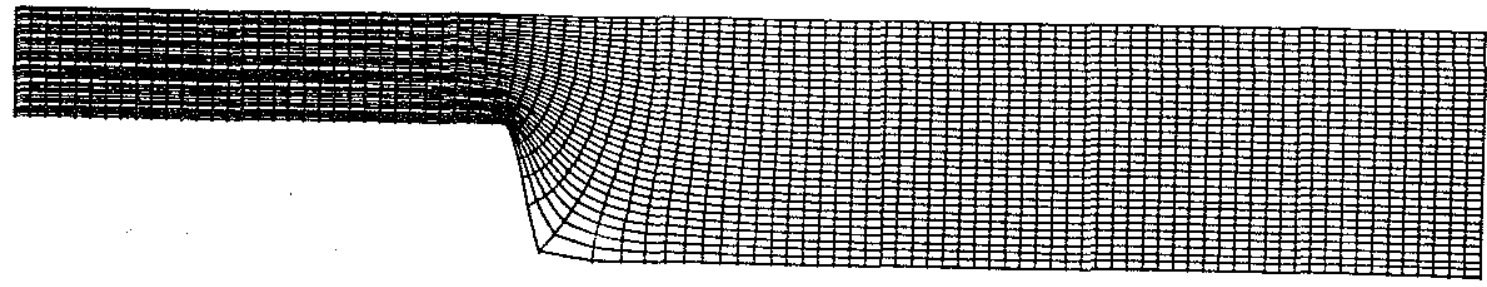

Figure 4-1: An example of the boundary-fitted orthogonal curvilinear coordinate grids used in the numerical experiments. The grid was computed using the algorithm described by Wilkin (1988).

The physical domain of the numerical experiments is typically, although not necessarily, bounded by an irregular coastline, a straight offshore boundary, and two straight across-shelf open boundaries. The computer code used in the present application to generate the curvilinear grids fitted to the domain (Wilkin, 1988) is based on an algorithm described by Ives and Zacharias (1987) which first maps the irregular geometry to a rectangle using repeated applications of a power (or hinge point) conformal transformation. Once the boundary mapping is complete the grid can be filled in by solving Laplace's equation for both the $x$ and $y$ coordinates. The metric factors $m, n$ are calculated from the computed grid according to (4.9) and (4.10) using centered differences. An example of one of the coordinate grids used in the experiments is shown in Figure 4-1.

\subsection{Coastal-Trapped Wave Across-Shelf Modal Structures}

In order to introduce free CTWs into the PEM, and to identify scattered CTWs in the model output, it is necessary first to compute their across-shelf modal structures. This section presents a synopsis of an algorithm (Wilkin, 1987) developed specifically for use in conjunction with the PEM which computes the modal structures of free CTWs for arbitrary bottom topography and stratification using the same numerical discretization scheme employed in the PEM.

The governing equations which result after making the approximations outlined in sec- 
tion 4.2 (linear, inviscid, hydrostatic, Boussinesq) are

$$
\begin{aligned}
\frac{\partial}{\partial t}\left(\frac{u}{m n}\right)-\frac{f}{m n} v & =-\frac{1}{n} \frac{\partial \phi}{\partial \xi} \\
\frac{\partial}{\partial t}\left(\frac{v}{m n}\right)+\frac{f}{m n} u & =-\frac{1}{m} \frac{\partial \phi}{\partial \eta} \\
\frac{\partial \phi}{\partial z} & =-\frac{\rho^{\prime} g}{\rho_{0}} \\
\frac{\partial \rho^{\prime}}{\partial t}+w \frac{\partial \rho}{\partial z} & =0 \\
m n\left(\frac{\partial}{\partial \xi}\left(\frac{u}{n}\right)+\frac{\partial}{\partial \eta}\left(\frac{v}{m}\right)\right)+\frac{\partial w}{\partial z} & =0
\end{aligned}
$$

where $u, v$ are the velocity components in the direction of the orthogonal curvilinear coordinates $\xi, \eta$ introduced in section 4.3. The density $\rho(x, y, z, t)$ has been further decomposed into a background vertical stratification $\bar{\rho}(z)$ and a perturbation due to the wave $\rho^{\prime}(x, y, z, t)$ so that the total density is now

$$
\rho_{\text {total }}=\rho_{0}+\bar{\rho}(z)+\rho^{\prime}(x, y, z, t)
$$

with $\rho_{0} \gg \bar{\rho} \gg \rho^{\prime}$.

Assuming solutions which are waves of fixed wavenumber $k$ propagating in the alongshelf $(\xi)$ direction with radian frequency $\omega$, equations (4.11) through (4.15) can be manipulated to give a single equation for the across-shelf modal structure of the pressure perturbation $\hat{\phi}(\eta, z)$ of a free cTw

$$
n \frac{\partial}{\partial \eta}\left(n \frac{\partial \hat{\phi}}{\partial \eta}\right)+\left(f^{2}-\omega^{2}\right) \frac{\partial}{\partial z}\left(\frac{1}{N^{2}} \frac{\partial \hat{\phi}}{\partial z}\right)-k^{2} \hat{\phi}=0
$$

where $N^{2}(z)=-\frac{g}{\rho_{0}} \frac{\partial \bar{\rho}}{\partial z}$ is the square of the Brunt-Väisälä frequency, assumed to vary only with $z$. The boundary conditions on (4.17) are:

- no flow through the coast or the solid wall placed at the offshore extent of the numerical domain $\left(v=0\right.$ at $\left.\eta=0, \eta_{\max }\right)$

- no vertical velocity at the rigid lid ( $w=0$ at $z=0$ )

- no flow through the bottom $\left(n \frac{\partial h}{\partial \eta} v+w=0\right.$ at $\left.z=-h\right)$

where the depth $h$ is assumed to vary in $\eta$ only. Stated in terms of the pressure $\hat{\phi}$ these boundary conditions are

$$
n \frac{\partial \hat{\phi}}{\partial \eta}+\frac{f k}{\omega} \hat{\phi}=0 \quad \text { at } \quad \eta=0, \eta_{\max }
$$




$$
\begin{aligned}
& \frac{\partial \hat{\phi}}{\partial z}=0 \text { at } \quad z=0 \\
& n \frac{\partial h}{\partial \eta}\left(n \frac{\partial \hat{\phi}}{\partial \eta}+\frac{f k}{\omega} \hat{\phi}\right)+\frac{f^{2}-\omega^{2}}{N^{2}} \frac{\partial \hat{\phi}}{\partial z}=0 \quad \text { at } \quad z=-h
\end{aligned}
$$

For a given $h, N^{2}, f$ and $k$ there is a sequence of solutions to (4.17) through (4.20) at discrete eigenfrequencies $\omega_{1}, \omega_{2}, \omega_{3} \ldots$ which are free CTWs (e.g. Huthnance, 1978).

This eigenvalue problem is solved by the method of resonance iteration in the following manner. At each iteration a guess is made for the free-wave wavenumber $(k)$ corresponding to the prescribed frequency $(\omega)$. The equations, after transformation to sigma coordinates, are discretized using the same numerical scheme employed in the PEM, i.e. horizontal finite-differences and a vertical expansion in Chebyshev polynomials. The resulting set of simultaneous linear equations is nearly singular if the chosen $k, \omega$ pair is close to that of a free CTW. By repeatedly solving the difference equations for different trial values of $k$ until a near resonant response to weak forcing occurs, a free-wave $k, \omega$ pair is found. The solution for $\hat{\phi}$ at this wavenumber is the across-shelf modal structure of the pressure perturbation of the free wave. The mode number will be the number of zero crossings in $\hat{\phi}$ along the bottom. The modal structures of the other variables are calculated from $\hat{\phi}$. The manner in which these computed modal structures are used in the initialization and analysis of the numerical experiments is outlined in the next section. Alternatively, the wavenumber $k$ can be prescribed and resonance iteration employed to compute the corresponding $\omega$. By repeating this calculation for many prescribed values of $k$, the dispersion relation $(\omega(k))$ of a free CTW can be computed. This procedure is described in greater detail by Wilkin (1987).

\subsection{The Numerical Wavetank}

The numerical experiments were conducted by combining the model components described in the preceding sections into what is best described as a 'numerical wavetank' (Figure 4-2). The wavetank is enclosed by four boundaries. Along the coastal wall a boundary condition of no normal flow is applied, this being appropriate for the study of inviscid CTws. For computational convenience, the offshore boundary is also treated as a free-slip wall. The wall is placed well offshore - typically 2 to 3 times the shelf width - and moving it farther offshore produces 


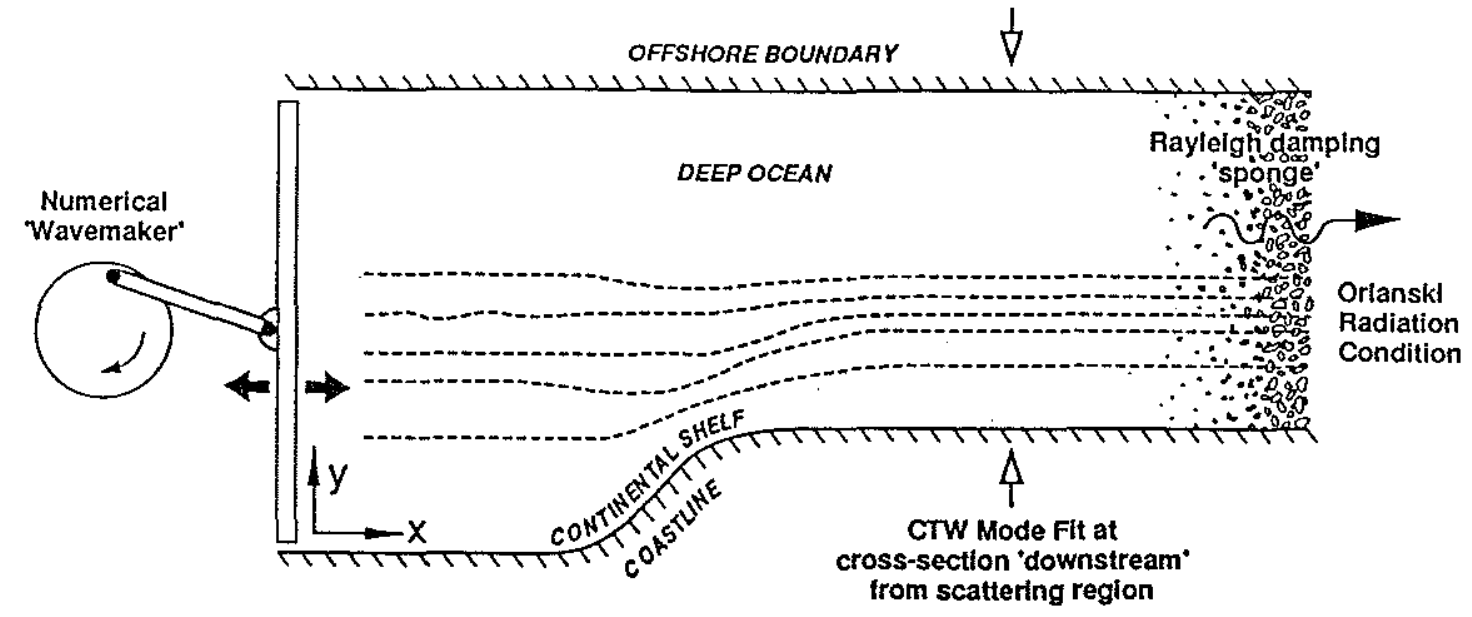

Figure 4-2: The 'numerical wavetank' configuration of the numerical model. The tank is bounded by free-slip lateral walls. A free coastal-trapped wave is introduced by a wavemaker at the upstream (left) end of the tank. Scattered waves propagate out through the radiation boundary at the downstream end of the tank.

negligible change in the $\mathrm{CTW}$ dispersion curves. Therefore, little difference in the numerical CTW scattering results would be obtained if a more realistic offshore boundary condition were employed. The incident wave is introduced into the computational domain by a numerical 'wavemaker' which prescribes $u, v$ and $\rho$ at each time step at the upstream across-shelf boundary. The prescription is a simple harmonic time-modulation of the incident-wave modal structures. The simulations begin with a quiescent wavetank and, to minimize initial transients, the wavemaker amplitude increases smoothly over one wave period to a constant value. As the wave propagates through the width change it scatters into a set of transmitted waves of different modes. An Orlanski (1976) radiation condition combined with a Rayleigh damping sponge at the downstream across-shelf boundary allows the scattered waves to propagate out of the computational domain with little reflection.

The horizontal spatial resolution of the model is set when the curvilinear coordinate grid is generated. The model runs reported in Chapter 5 were conducted with an alongshelf grid spacing of approximately $15 \mathrm{~km}$, an across-shelf spacing of 4 to $6 \mathrm{~km}$, and 7 Chebyshev poly- 
nomials in the vertical. Tests of CTW propagation in a straight channel with periodic open boundary conditions showed that for spatial resolution of this order, root-mean-square errors in the horizontal velocity components after one wave period were at most a few percent for the low modes (Haidvogel et al., 1988).

Laplacian diffusion applied on constant sigma surfaces was employed to damp out the smallscale (two grid point) computational noise which arises in the simulations. The Laplacian operator includes both alongshelf $(\xi)$ and across-shelf $(\eta)$ terms. It was found that, by using a large diffusion coefficient $\left(500 \mathrm{~m}^{2} \mathrm{~s}^{-1}\right)$ for the $\xi$ term and a smaller value $\left(100 \mathrm{~m}^{2} \mathrm{~s}^{-1}\right)$ for the $\eta$ term, the noise could be controlled without affecting significantly the propagation of the CTws. No damping was applied to the density equation.

\subsection{Analysis: Modal Decomposition}

In order to understand the simulated CTW scattering it is desirable to extract the amplitudes of the scattered modes from the PEM output. This is achieved by performing a least squares fit of the model output to a sum of propagating CTW modes downstream from the scattering region. Evanescent motions trapped at the scattering region have an alongshelf decay scale comparable to the across-shelf scale of the topography or the lowest deep-ocean internal Rossby radius, whichever is greater. In practice, the topographic length scale almost always dominates, so provided the modal fit is performed 200 to $300 \mathrm{~km}$ downstream of the scattering region, the motions present ought to be due solely to freely propagating scattered cTws.

The fit is performed to the alongshelf velocity which has a strong signal for all stratifications and topographies. Let $\xi_{0}$ be the alongshelf location at which the fit is performed. At $\xi_{0}$ the grid must be rectangular and the topography must vary only with $\eta$. The alongshelf velocity field calculated by the PEM is represented as

$$
u_{\mathrm{PEM}}\left(\xi_{0}, \eta, z, t\right)=\sum_{q=1}^{Q} B_{q}\left(\xi_{0}, t\right) U_{q}(\eta, z)+\varepsilon\left(\xi_{0}, \eta, z, t\right)
$$

where $U_{q}(\eta, z)$ is the modal structure of the alongshelf velocity of transmitted mode $q$ and $B_{q}\left(\xi_{0}, t\right)$ is the instantaneous amplitude of mode $q$ at time $t$. The residual error between the CWW mode fit and the PEM output is $\varepsilon\left(\xi_{0}, \eta, z, t\right)$. A total of $Q$ transmitted modes is included in the analysis. Once the initial transients have passed, and provided the section $\xi_{0}$ is not too 
close to the Rayleigh sponge, the modal decomposition returns time series of $B_{q}\left(\xi_{0}, t\right)$ values which are periodic with the frequency of the incident wave.

Integrating $\varepsilon^{2}$ over the vertical across-shelf plane at $\xi_{0}$ gives the square of the total residual error in the mode fit:

$$
R^{2}=\int_{\eta=0}^{\eta_{\mathrm{tnax}}} \int_{z=-h}^{0}\left(u_{\mathrm{rEM}}\left(\xi_{0}, \eta, z, t\right)-\sum_{q=1}^{Q} B_{q}\left(\xi_{0}, t\right) U_{q}(\eta, z)\right)^{2} d z d \eta
$$

Minimizing $R^{2}$ (by setting $\partial R^{2} / \partial B_{m}=0$ for $m=1,2 \ldots Q$ ) gives a set of simultaneous equations for the time series of the mode amplitudes:

$$
\frac{\partial R^{2}}{\partial B_{m}}=\int_{0}^{\eta_{\max }} \int_{-h}^{0}-2 U_{m}(\eta, z)\left(u_{\mathrm{PEM}}\left(\xi_{0}, \eta, z, t\right)-\sum_{q=1}^{Q} B_{q}\left(\xi_{0}, t\right) U_{q}(\eta, z)\right) d z d \eta=0
$$

which may be written

$$
\sum_{q=1}^{Q} A_{m q} B_{q}\left(\xi_{0}, t\right)=r_{m} \quad m=1,2 \ldots Q
$$

where

$$
A_{m q}=\int_{0}^{\eta_{\max }} \int_{-h}^{0} U_{m}(\eta, z) U_{q}(\eta, z) d z d \eta
$$

and

$$
r_{m}=\int_{0}^{\eta_{\mathrm{rax}}} \int_{-h}^{0} U_{m}(\eta, z) u_{\mathrm{PBM}}\left(\xi_{0}, \eta, z, t\right) d z d \eta
$$

In practice, the integrations over $\eta$ and $z$ in (4.25) and (4.26) are performed numerically.

The reader may wish to look ahead to Figure 4-3, an example of the modal fit results, to see that the $B_{q}\left(\xi_{0}, t\right)$ time series do indeed become periodic once the initial transients have passed. The magnitude of each harmonic series gives the amplitude of the corresponding CTW mode. The normalization of the $U_{q}(\eta, z)$ modal structures is performed in such a manner that the energy flux of each mode, integrated across the shelf, is given simply by the amplitude squared.

\subsection{Model Performance Test: Comparison with Analytical Results}

By generating an orthogonal curvilinear coordinate grid fitted to a coastal channel whose width increases abruptly, it has been possible to conduct a test of the numerical wavetank configuration 
of the PEM, and the modal decomposition method, by running a simulation of BSW scattering which nearly duplicates the problem considered in Chapter 3 .

The grid used in the simulation is that shown in Figure 4-1. It differs slightly from the problem considered in Chapter 3 in that, rather than being discontinuous, the channel width increases smoothly over a distance of $50 \mathrm{~km}$. A step width change in the numerical grid would produce extremely small grid spacings in at least one region of the domain and place a prohibitive constraint on the numerical model time-step. The particular case simulated is that of Table 3-1. The incident wave is mode 1 , the frequency is $\omega=1 \times 10^{-5} \mathrm{~s}^{-1}(0.1 \mathrm{f})$, the shelf widths are $100 \mathrm{~km}$ and $225 \mathrm{~km}$, and the exponential topography has a decay scale of $\lambda=1 \times 10^{-2} \mathrm{~km}^{-1}$. For these values the analytical solution shows that a shadow zone of weak flow extends roughly $100 \mathrm{~km}$ downstream from the width change (Figure 3-8). Therefore, the $50 \mathrm{~km}$ long region of changing channel width in the numerical simulation should be within the shadow zone, and the numerical and analytical results should not differ appreciably.

The incident wave was introduced into the numerical domain in the manner described in section 4.5. The time series of the transmitted mode amplitudes computed by a least-squares mode fit performed $200 \mathrm{~km}$ downstream from the scattering region are shown in Figure 4-3. Shortly after the scattered waves reach the alongshelf location where the mode fit is performed the time series become periodic with the frequency of the incident wave, as they ought (section 4.6). The dashed line in Figure $4-3$ is a time series of the residual error of the mode fit ( $R$ of equation 4.22 ) normalized by the total root-mean-squared velocity. Such a small residual error shows that the alongshelf velocity downstream from the scattering region is represented well by a set of freely propagating Bsws. The magnitudes of the time series in Figure 4-3 are $0.549,0.687,0.428$ and 0.108 for modes 1 through 4 respectively, which correspond to energy fluxes of $0.302,0.470,0.183$ and $1.17 \times 10^{-2}$. The energy in the higher modes is negligible. The total transmitted energy flux measured by the mode fit is therefore 0.967 , which differs by $3 \%$ from the analytical value of 1.0 . This error level is comparable to that determined by Haidvogel et al. (1988) in their tests of $\mathrm{Crw}$ propagation in a straight periodic channel version of the PEM.

Before comparing the individual mode amplitudes to the analytical solution, the values computed by the mode fit must be adjusted to account for the different normalization method 


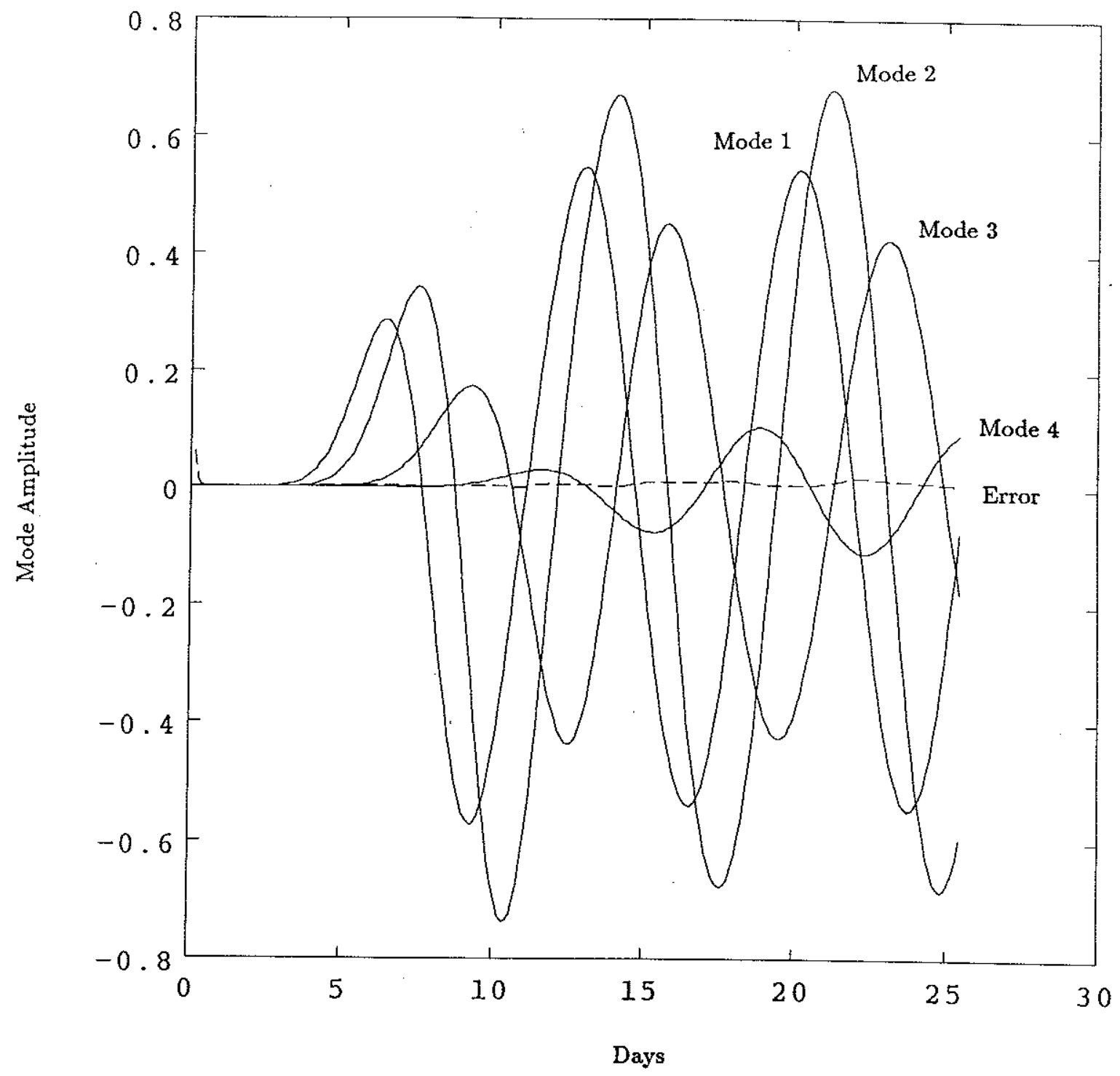

Figure 4-3: Time series of the amplitudes of the transmitted modes returned by the least-square mode fit for a simulation of the BSW scattering problem considered in Chapter 3. Dashed line is the residual error ( $R$ of equation 4.22). 
used in Chapter 3. (In the numerical simulations the modal structures are normalized such that the energy flux is simply the square of the amplitude, whereas in Chapter 3 the energy fluxes are given by equation (3.37)). The renormalized amplitudes for the mode fit results are 0.365 , $0.457,0.286$ and 0.073 , which compare favorably with the analytical values of $0.346,0.461,0.316$ and 0.085 , respectively (Table $3-1$ ). The discrepancy is consistent with the smoother coastline change being less conducive to scattering into higher modes.

From this test, it is apparent that the numerical wavetank configuration of the PEM can be applied reliably to the simulation of BSw scattering by irregular coastal geometry, and that the modal fit method correctly extracts the mode amplitudes from the model output. Other tests of the PEM (Haidvogel et al., 1988) show that the model correctly propagates CTws in a stratified coastal ocean. There is every indication therefore, that the model can be used with confidence in the simulation of CTW scattering processes. 


\section{Chapter 5}

\section{Numerical Results and Discussion}

\subsection{CTW Dispersion Properties and Scattering Regimes}

As is the case with many other geophysical wave phenomena, the dispersion properties of free CTWs play a key role in their behavior as they propagate through an irregular waveguide. Indeed, for ctws it is possible to deduce several net energy transmission properties of a particular scattering geometry by simply examining the dispersion curves of free crws at each end of the scattering region without detailed consideration of the dispersion properties within the scattering region itself.

Consider the two across-shelf depth profiles (Figure 5-1), which are similar qualitatively to many continental shelves. The dispersion curves of the mode 1 CTWs for these two topographies, for different stratifications (i.e. different constant values of $N^{2}$ ), are shown in Figure 5-2. The curves, computed using the algorithm of section 4.4 , illustrate the theoretical result derived by Huthnance (1978) that increasing the stratification increases the wave frequency at all wavenumbers. Another result derived by Huthnance (1978) is that as the wavenumber ( $k$ ) becomes large the frequency $(\omega)$ tends to a constant value provided the bottom slope remains bounded. This limiting frequency is given by

$$
\omega_{\infty}=\lim _{k \rightarrow \infty} \omega=\max _{y}\left[\left.N\right|_{z=-h(y)} \frac{d h}{d y}\right]
$$

where $y$ is the across-shelf coordinate. The frequency of every mode converges to this limit.

At low stratifications, $\omega(k)$ has at least one stationary point $\omega_{c}$ where $\omega$ takes a local 


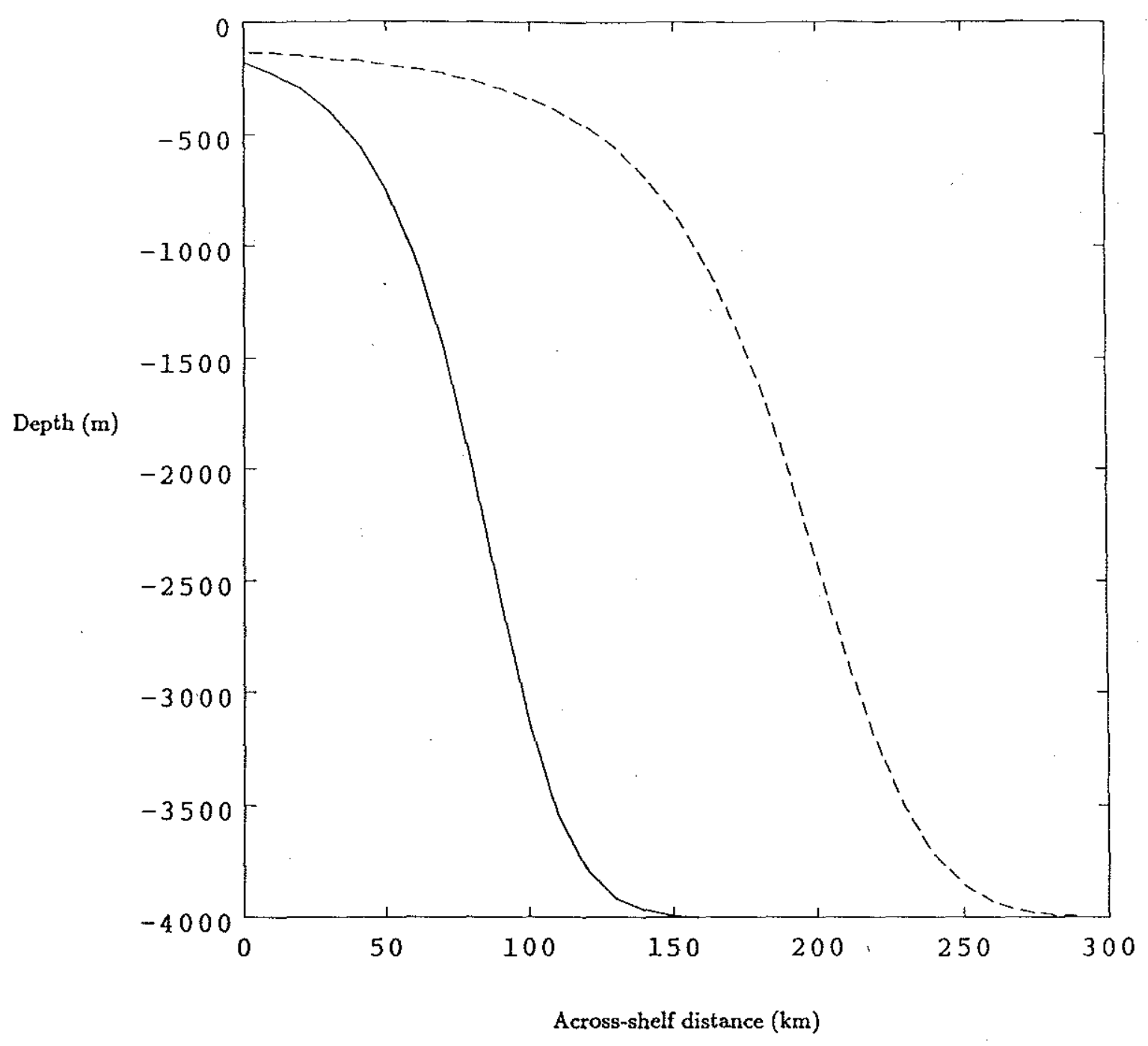

Figure 5-1: Across-shelf depth profiles on which the dispersion curves of Figure 5-2, and the scattering regime diagrams (Figure 5-3), are based. 

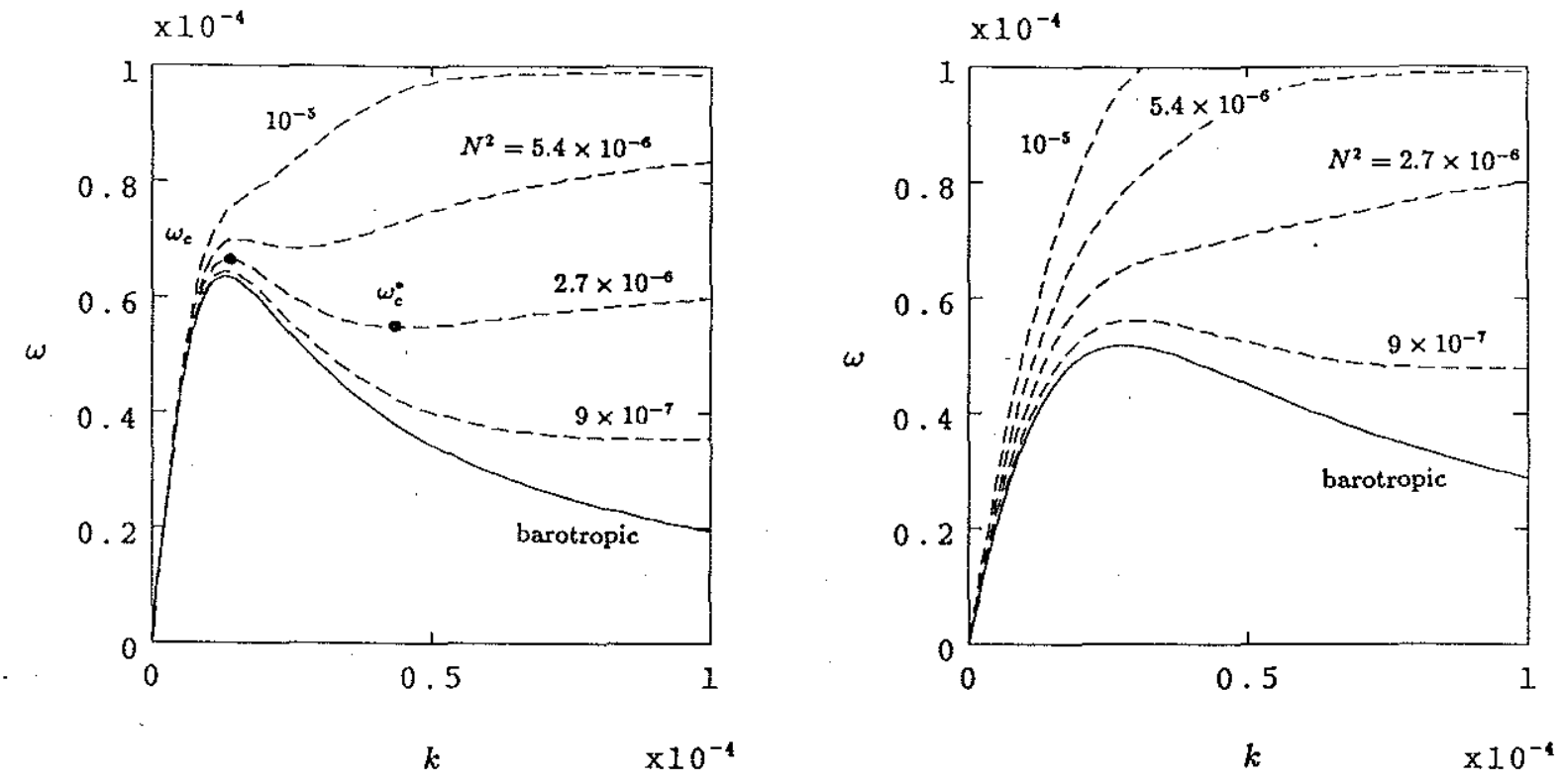

Figure 5-2: Mode $1 \mathrm{CTW}$ dispersion curves for the (left) wide and (right) narrow topographies of Figure 5-1 computed for different constant values of $N^{2}$, for $f=10^{-4} s^{-1}$. Curves are labelled with $N^{2}$ values in units $s^{-2}$. The stationary points of $\omega(k)$ defined in the text, i.e. $\omega_{c}$ and $\omega_{c}^{*}$, are illustrated on the $N^{2}=2.7 \times 10^{-6} s^{-2}$ curve in the left panel.

maximum. There is therefore a range of frequencies for which cTws with negative group velocity can exist. Within this class, there is a range of stratifications for which $\omega(k)$ may also take a local minimum, $\omega_{c}^{*}$, for one or more of the lowest modes. The value of $\omega_{c}^{*}$ for the highest mode with a local minimum frequency is the lowest frequency at which a CTw with negative group velocity can occur. If there are no local minima then the lower bound for negative group velocity waves is simply $\omega_{\infty}$. The maximum frequency at which a wave with positive group velocity is possible is given by the maximum of $\omega_{c}$ and $\omega_{\infty}$.

As the strength of the stratification increases, a qualitative change in the form of the curves occurs, from a class of dispersion curves which has a region of negative slope, to a second class of curves without any negatively sloping region. The second class of dispersion curves occurs at moderate to high stratification. In this case the curves increase monotonically to the limiting value $\omega_{\infty}$ so that there are no free waves above $\omega_{\infty}$ and no waves at all with negative group 


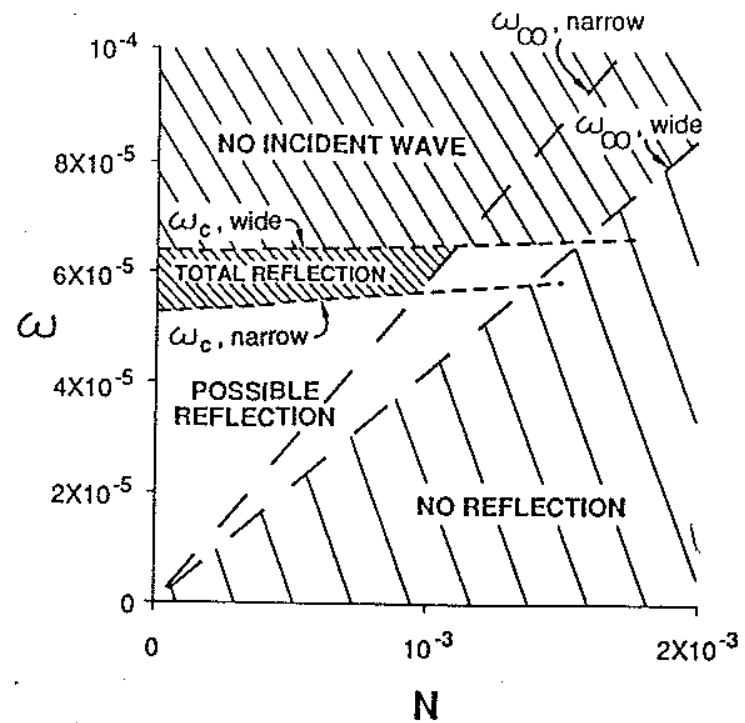

(a) Narrowing

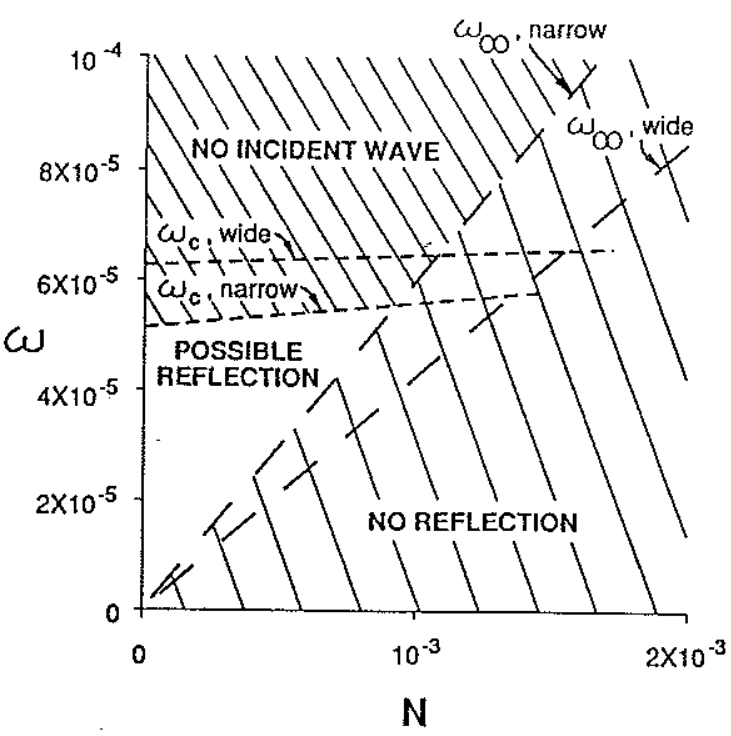

(b) Widening

Figure 5-3: Scattering regimes for shelf bathymetries which, in the direction of CTw propagation, make a transition from the (a) wide to the narrow, and (b) narrow to the wide, topographies of Figure 5-1.

velocity.

The significance of these limiting frequencies to $\mathrm{CTW}$ scattering processes becomes apparent when the limits for two different topographies are compared as functions of stratification. The solid and dashed lines in Figure 5-3 show the limiting frequencies for the wide and narrow topographies, respectively, of Figure 5-1. The $\omega_{c}$ values were taken directly from Figure 5-2 and $\omega_{\infty}$ was calculated from (5.1). The minimum $\omega_{c}^{*}$ values are shown only schematically since their evaluation would require the computation of dispersion curves for many modes at many stratifications. If, in the direction of CTW propagation, the shelf bathymetry makes a smooth transition from the wide to the narrow topography then the various frequency limits imply the existence of the scattering regimes shaded in Figure 5-3a. Consider a slice through Figure 5-3a at some constant low value of $N$ : At very high frequencies there are no waves with positive group velocity on the wider shelf - an incident wave cannot exist therefore, and the question of how it might scatter is moot. Below the wide shelf cutoff frequency $\left(\omega_{c, \text { wide }}\right)$, but above the 
narrow' shelf cutoff frequency $\left(\omega_{c, n a r r o w}\right)$, there is a range where an incident wave is possible but there are no waves with positive group velocity on the narrow shelf - therefore there are no waves able to transport energy into the region of narrow topography and the energy flux of the incident wave must be totally reflected. Below this range is a range where both incident and transmitted waves exist and partial reflection of CTW energy is possible though by no means assured. At still lower frequencies (i.e. $\omega<\min \left[\omega_{c, w i d e}^{*}, \omega_{\infty}\right.$,wide $]$ ) there are no waves with negative group velocity on the wider shelf - therefore there cannot be any reflection and all the incident wave energy flux must be scattered forward into the CTW modes of the narrow shelf. If the topographic transition is in the opposite sense, i.e. narrow to wide, each limiting frequency line assumes a different significance and the resulting scattering regimes are as shown in Figure 5-3b. In this case transmitted waves exist for all frequencies at which an incident wave is possible and the region of total reflection is no longer present.

The statistical analyses cited in Chapter 1 show that the energy of observed CTw motions is generally located in a band of frequencies corresponding to periods of a few days to a few weeks. In coastal oceans average values of $N^{2}$ are typically in the neighborhood of $10^{-6}$ to $10^{-5} s^{-2}$. For this range of parameters, i.e. $\omega<2 \times 10^{-5} s^{-1}, 10^{-3}<N<3 \times 10^{-3} s^{-1}$, inspection of Figure 5-3 suggests that regardless of whether the shelf widens or narrows, CTws should not be reflected by the topographic variation. This illustrates the basis for the assertion in the analytical study of Chapter 3 that it was appropriate to limit discussion to the range of parameters for which there was no reflection of BSW energy.

It should be emphasized at this point that Figure 5-3 is based on the topographies shown in Figure 5-1 and uniform stratifications ( $N$ independent of depth). For other topographies and stratification profiles, the boundaries of the scattering regimes are similar qualitatively but differ quantitatively due to differences in the details of the dispersion curves.

While this analysis is useful for identifying gross properties of the CTW scattering process such as total energy transmission or reflection, it provides no information about what proportion of the incident energy is scattered into each of the transmitted CTW modes. Nor is there any indication of how much reflection can be expected in the regions labelled "possible reflection". Furthermore, there is no consideration of the details of the flow field within the scattering region. To answer these questions it is necessary to proceed to the numerical experiments. 


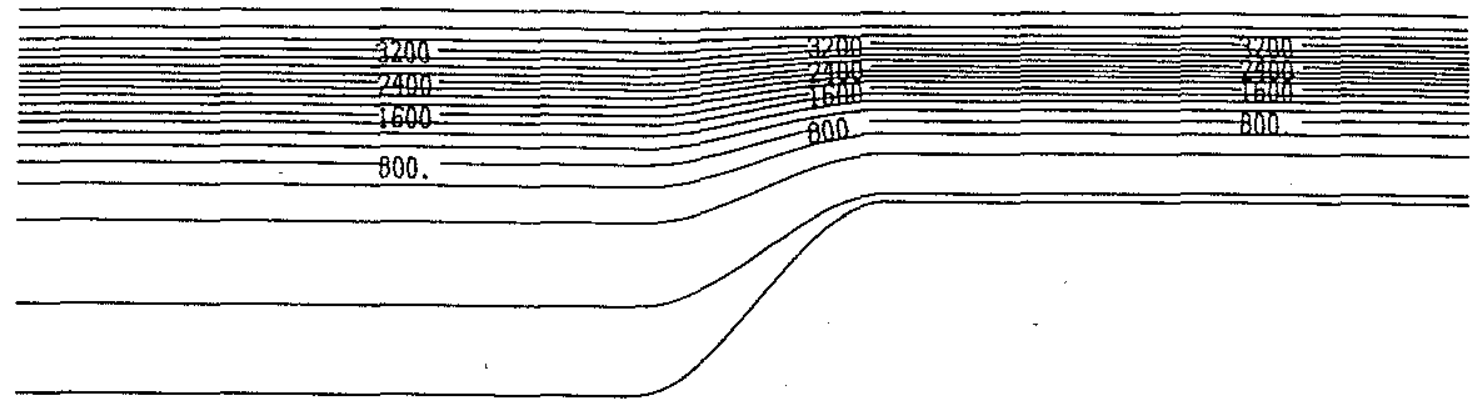

Figure 5-4: Bathymetry used in the first series of narrowing shelf scattering experiments. Contour interval is $200 \mathrm{~m}$. The depth profiles outside the scattering region are those of Figure $5-1$.

\subsection{Narrowing Shelf Results}

The first series of numerical simulations examines the scattering of CTWs by the bathmetry plotted in Figure 5-4. The depth profiles outside the region of converging isobaths are those of Figure 5-1. The shelf narrows by $125 \mathrm{~km}$ over an alongshelf distance of $200 \mathrm{~km}$ with most of the variation occuring at the coast while the continental slope remains relatively straight. The case of a mode 1 incident wave with frequency $1 \times 10^{-5} s^{-1}(0.1 f)$ and uniform stratification $N^{2}=2.7 \times 10^{-6} \mathrm{~s}^{-2}$, is in the "no reflection" regime of Figure 5-3a, so the energy flux in the transmitted waves should balance the incident energy flux. The magnitudes of the least squares mode fit time series for this case, computed as described in section 4.6 , are $0.973,0.309$ and 0.125 for modes 1 through 3 respectively, which give energy fluxes of $0.948,0.096$ and 0.016 and a total transmitted energy flux of 1.065 . The energy in the higher modes is negligible. Since the incident wave has unit energy flux it is apparent that the model overestimates the energy of the scattered waves by some $6 \%$. A $6 \%$ error in energy corresponds to roughly $3 \%$ errors in the mode amplitudes. This error level is comparable to that determined by Haidvogel et al. (1988) in their tests of CTW propagation in a straight periodic channel version of the PEM.

The effect that stratification has on the strength of the scattering was examined by repeating 


\begin{tabular}{ccccc}
\hline \multirow{2}{*}{ Mode } & & $N^{2}\left(s^{-2}\right)$ & \\
& 0 & $9 \times 10^{-7}$ & $2.7 \times 10^{-6}$ & $5.4 \times 10^{-6}$ \\
& & & & \\
\hline 1 & 0.932 & 0.915 & 0.890 & 0.877 \\
2 & 0.060 & 0.074 & 0.090 & 0.094 \\
3 & $4.4 \times 10^{-3}$ & 0.019 & 0.015 & 0.017 \\
4 & - & $7.6 \times 10^{-4}$ & $5.1 \times 10^{-3}$ & $9.3 \times 10^{-4}$ \\
& & $(1.069)$ & $(1.065)$ & $(1.067)$ \\
\hline
\end{tabular}

Table 5-1: Energy fluxes of the transmitted modes generated when a mode 1 incident wave with frequency $1 \times 10^{-5} s^{-1}$ encounters the variable topography of Figure 5-4 for different values of uniform stratification $\left(N^{2}\right)$. More energy is lost from mode 1 (i.e. scattering increases) as the stratification increases. The total transmitted energy flux computed by each modal fit and used to normalize the other table entries (see text) is shown in parentheses in the final row.

the above simulation for several values of $N^{2}$ (Table 5-1). In all cases the modal fit overestimated the total transmitted energy by $5 \%$ to $7 \%$, so the individual transmitted mode energy fluxes have been normalized by the modal fit total energy flux. This should bring the fluxes more closely into line with their true values. For completeness, the total energy flux values returned by the modal fit are shown in the bottom row of Table 5-1. The differences between these values and unity indicate the accuracy of the other entries in the table.

The results show clearly that increasing stratification amplifies the scattering process - the energy flux remaining in mode 1 diminishes with increasing stratification while that in mode 2 increases. Overall however, the strength of the scattering is not pronounced, especially in the barotropic $\left(N^{2}=0\right)$ case where the incident mode loses only $7 \%$ of its energy flux. This may seem surprising at first, given the large change in shelf width. It is less surprising when the idea of "shelf-similarity," which was introduced by Hsueh (1980) and Davis (1983) (section 2.5), is recalled. A shelf-similar topography will not scatter long barotropic shelf waves, and the bathymetry used in this series of experiments (Figure 5-4) departs only moderately from the definition of shelf-similarity given by Hsueh (1980); namely, that the distance from each isobath to the shore is a fixed fraction of the local shelf width. The next series of scattering experiments 


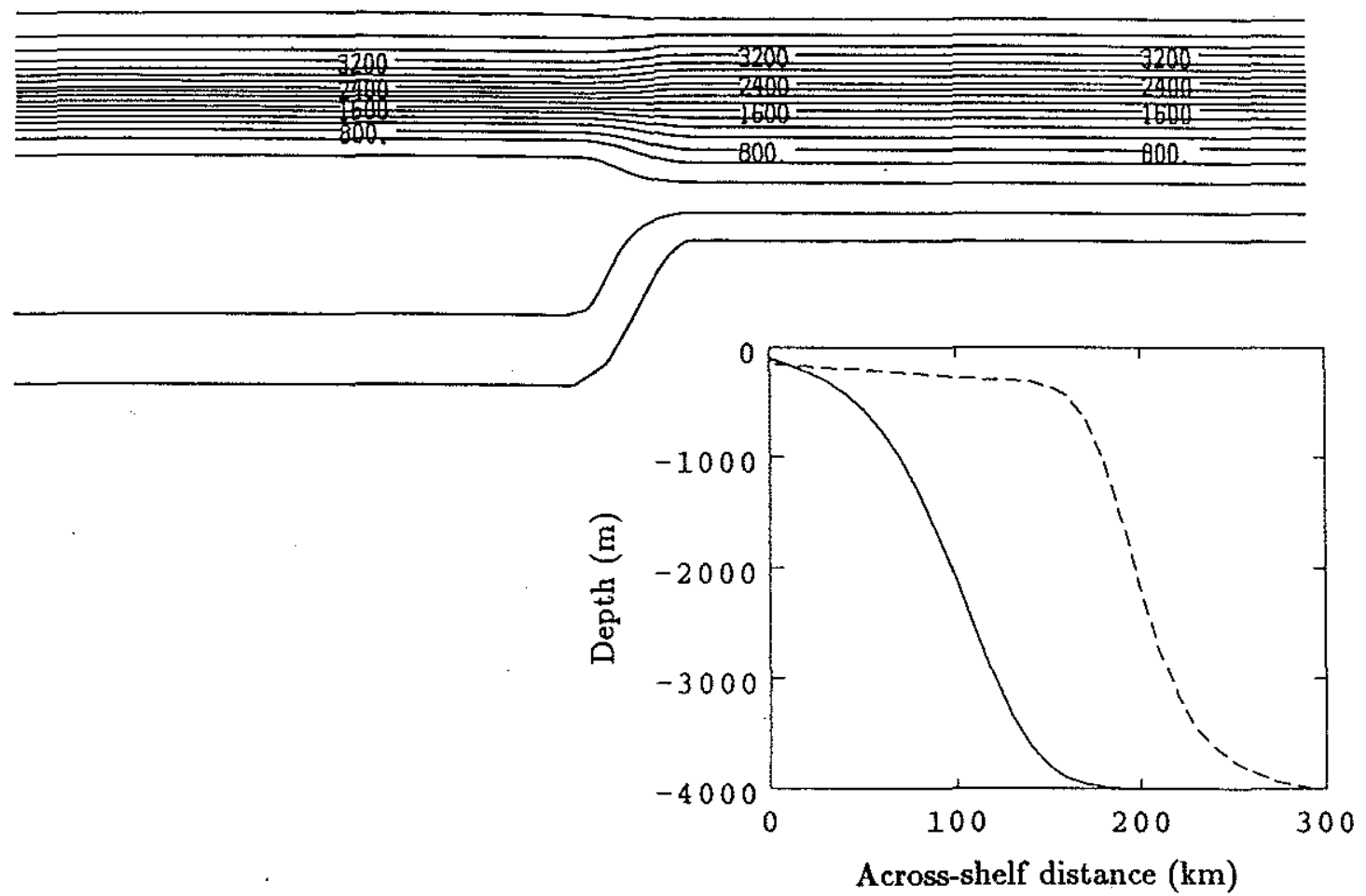

Figure 5-5: Bathymetry used in the second series of narrowing shelf scattering experiments. Contour interval is $200 \mathrm{~m}$. The depth profiles outside the scattering region are shown inset.

was designed to examine CTW scattering by a much less shelf-similar topography.

\section{Less Shelf-Similar Topography}

The bathymetry used in the second series of simulations is plotted in Figure 5-5. In this case the depth profiles outside the scattering region are markedly different, in contrast to the previous series of experiments. The wider shelf has a broad region which slopes gently away from the coast but drops off abruptly to abyssal depths, whereas the narrower shelf is more uniformly sloping with a less pronounced shelf break. Consequently, as the shelf narrows over an alongshelf distance of $100 \mathrm{~km}$ (i.e. more abruptly than in the first series of experiments) 


\begin{tabular}{ccccc}
\hline \multirow{4}{*}{ Mode } & \multicolumn{4}{c}{$N^{2}\left(s^{-2}\right)$} \\
& 0 & $9 \times 10^{-7}$ & $2.7 \times 10^{-6}$ & $5.4 \times 10^{-6}$ \\
\cline { 2 - 5 } & & & & \\
\hline & & & 0.803 & 0.795 \\
2 & 0.877 & 0.847 & 0.182 & 0.169 \\
3 & $4.7 \times 10^{-3}$ & $4.7 \times 10^{-3}$ & 0.014 & 0.031 \\
4 & $6.3 \times 10^{-3}$ & $1.5 \times 10^{-3}$ & $2.9 \times 10^{-4}$ & $4.8 \times 10^{-3}$ \\
& & & & \\
& $(1.037)$ & $(1.046)$ & $(1.040)$ & $(1.032)$ \\
\hline
\end{tabular}

Table 5-2: Energy fluxes of the transmitted modes generated when a mode 1 incident wave of frequency $1 \times 10^{-5} s^{-1}$ encounters the variable topography of Figure 5-5 for different values of the stratification $\left(N^{2}\right)$. Table entries are calculated in the same manner as Table 5-1. Scattering is stronger (i.e. more energy is lost from the incident mode) than for the topography of Figure 5-4.

some isobaths are displaced shoreward while others move offshore. This bathymetry therefore departs strongly from Hsueh's (1980) definition of shelf-similarity. In a series of experiments analogous to the first series, the scattering of a mode 1 incident wave with frequency $1 \times 10^{-5} s^{-1}$ was examined for a range of stratifications. The results, in terms of transmitted mode energy fluxes, are presented in Table 5-2 in the same manner as the results in Table 5-1. As in the previous experiments, stratification amplifies the strength of the scattering. Comparison with Table 5-1 shows that at all stratifications the scattering is more pronounced for this less-shelfsimilar topography - more than $20 \%$ of the incident mode energy flux is transferred to higher modes when the stratification reaches the still modest level of $N^{2}=5.4 \times 10^{-6} \mathrm{~s}^{-2}$.

More insight into the role played by stratification in modifying the scattering process can be gained by examining scattering by an exactly shelf-similar topography.

\section{Shelf-Similar Topography}

As noted in section 2.5, barotropic shelf waves should propagate through shelf-similar topographic variations without scattering. However, with the introduction of stratification this property may no longer hold, and scattering may occur. The third series of scattering experiments considers the scattering of a CTW by a topography (Figure $5-6$ ) which is exactly 


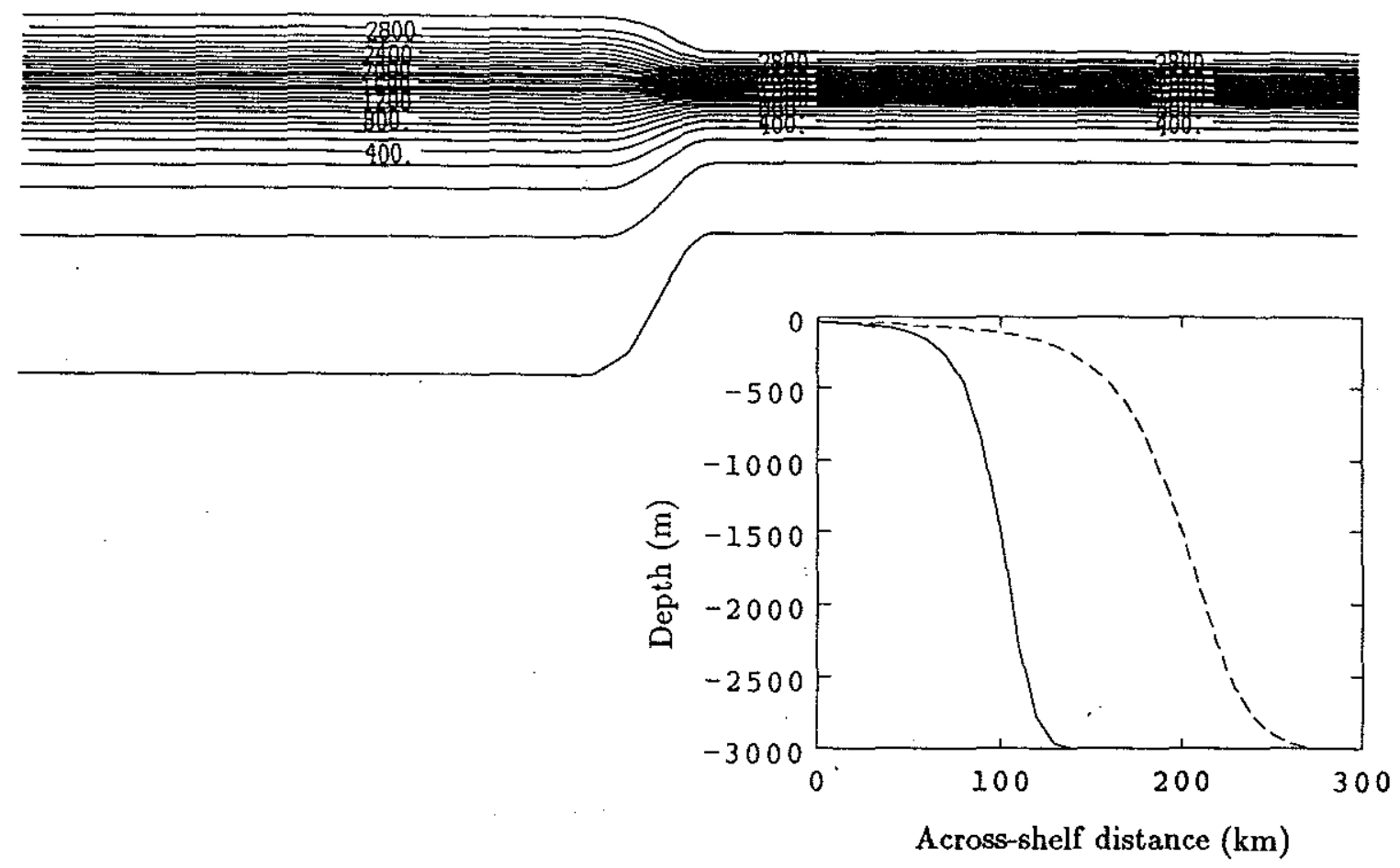

Figure 5-6: Shelf-similar bathymetry used in the third series of narrowing shelf scattering experiments. Contour interval is $100 \mathrm{~m}$. The depth profiles outside the scattering region are shown inset.

shelf-similar in the sense described by Hsueh (1980).

As in the previous two series of experiments, the incident wave is mode 1 and has a frequency of $1 \times 10^{-5} s^{-1}$. At all the stratifications considered there is practically no scattering of energy into modes other than that of the incident wave (Table 5-3), even for stratifications stronger than those considered previously. Therefore, the introduction of stratification does not, of itself, appear to cause CTWs to scatter on a shelf where scattering is not induced in the barotropic limit. These results suggest that the role of stratification in CTW scattering processes is one of modifying the scattering triggered by non-shelf-similar topographic irregularities. 


\begin{tabular}{cccccc}
\hline Mode & \multicolumn{5}{c}{$N^{2}\left(s^{-2}\right)$} \\
& 0 & $1.8 \times 10^{-6}$ & $2.7 \times 10^{-6}$ & $5.4 \times 10^{-6}$ & $1 \times 10^{-5}$ \\
& & & & & \\
\hline & & & & & \\
1 & 0.993 & 0.994 & 0.993 & 0.988 & 0.982 \\
2 & $2.8 \times 10^{-3}$ & $2.1 \times 10^{-3}$ & $2.9 \times 10^{-3}$ & $7.7 \times 10^{-3}$ & 0.016 \\
3 & $3.4 \times 10^{-3}$ & $3.5 \times 10^{-3}$ & $3.4 \times 10^{-3}$ & $2.0 \times 10^{-3}$ & $9.6 \times 10^{-4}$ \\
4 & - & $4.1 \times 10^{-4}$ & $7.2 \times 10^{-4}$ & $1.5 \times 10^{-3}$ & $1.6 \times 10^{-3}$ \\
& & & & & \\
& $(1.057)$ & $(1.063)$ & $(1.061)$ & $(1.062)$ & $(1.048)$ \\
\hline
\end{tabular}

Table 5-3: Energy fluxes of the transmitted modes generated when a mode 1 incident wave of frequency $1 \times 10^{-5} s^{-1}$ encounters the shelf-similar topography of Figure 5-6 for different values of the stratification $\left(N^{2}\right)$. Table entries are calculated in the same manner as Table 5-1. For this topography there is no appreciable scattering at any stratification.

\section{Coastline Effects}

The irregular topographies considered up to this point can be characterized as abrupt narrowings of the shelf due primarily to variations in the position of the coastline. In all cases the continental slope remains relatively straight. The next series of simulations addresses the question of whether the form of the transition between two regions of straight shelf affects the strength of CTW scattering.

The vorticity gradient which contributes to the support of CTWs is greatest where the across-shelf bottom slope is steepest. It is probable therefore, that variations in the position of the continental slope act as more severe irregularities in the CTW waveguide than comparable displacements of the coastline. The following series of experiments confirms that this is the case.

In this set of simulations the depth profiles outside the scattering region are identical to those of the shelf shown in Figure 5-5 but the transition between the two profiles, which occurs over an alongshelf distance of $100 \mathrm{~km}$, is achieved without any change in the position of the coast (Figure 5-7). The energy fluxes in the transmitted modes, for an incident mode 1 wave with frequency $1 \times 10^{-5} s^{-1}$, are presented in Table 5-4. As expected, the incident mode loses somewhat more energy in this case than in the corresponding straight-continental-slope case 


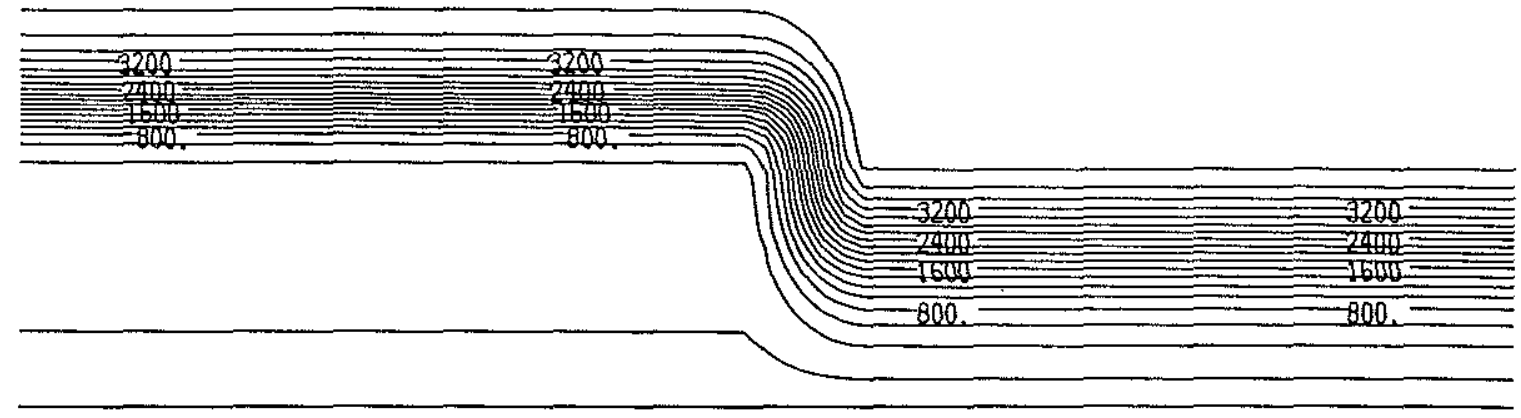

Figure 5-7: Bathymetry used in the fourth series of narrowing shelf scattering experiments. Contour interval is $200 \mathrm{~m}$. The depth profiles outside the scattering region are the same as those shown inset in Figure 5-5.

(Table 5-2).

An additional run was made for the $N^{2}=2.7 \times 10^{-6} s^{-2}$ case with the alongshelf distance separating the two different shelf profiles (i.e. the length of the scattering region) increased to $200 \mathrm{~km}$. This doubling of the length of the scattering region produced changes of less than $1 \%$ in the energy fluxes of scattered modes 1 and 2 . This suggests that the strength of the scattering induced by shelf width changes is not sensitive to the alongshelf length of the scattering region for lengths such as those considered here which are much less than a CTW wavelength.

\section{Scattering Coefficient}

The scattering properties of the four topographies considered in the simulations above can be compared by defining a scattering coefficient $C_{s c}$ :

$$
C_{s c}=1-\frac{\text { Energy flux in transmitted mode } 1}{\text { Total transmitted energy flux }}
$$

If the incident mode 1 wave passes through the scattering region without loss of energy then $C_{s c}=0$, and if the incident mode loses energy to other transmitted modes, $C_{s c}$ is greater than 


\begin{tabular}{cccccc}
\hline \multirow{5}{*}{ Mode } & \multicolumn{5}{c}{$N^{2}\left(s^{-2}\right)$} \\
& 0 & $9 \times 10^{-7}$ & $2.7 \times 10^{-6}$ & $5.4 \times 10^{-6}$ & $1 \times 10^{-5}$ \\
& & & & & \\
\hline & & & & & \\
1 & 0.854 & 0.800 & 0.758 & 0.746 & 0.730 \\
2 & 0.139 & 0.181 & 0.209 & 0.213 & 0.194 \\
3 & $6.9 \times 10^{-3}$ & 0.017 & 0.025 & 0.037 & 0.052 \\
4 & $1.8 \times 10^{-8}$ & $1.7 \times 10^{-3}$ & $7.4 \times 10^{-3}$ & $4.5 \times 10^{-3}$ & 0.024 \\
& & & & & \\
& $(1.013)$ & $(1.028)$ & $(1.020)$ & $(1.016)$ & $(0.967)$ \\
\hline
\end{tabular}

Table 5-4: Energy fluxes of the transmitted modes generated when a mode 1 incident wave of frequency $1 \times 10^{-5} s^{-1}$ encounters the variable topography of Figure 5-7 for different values of the stratification $\left(N^{2}\right)$. Table entries are calculated in the same manner as Table $5-1$. The shelf profiles outside the scattering region are the same as for the case considered in Table 5-2 but the scattering is stronger here because the transition between the two profiles occurs primarily as a displacement of the shelf/slope break.

zero. In Figure 5-8, $C_{s c}$ is plotted against $N^{2}$ for the four topographies considered. The curves show similar trends suggesting that a simple relationship may exist for $C_{s c}$ as a function of stratification.

Recalling the earlier result that the effect of stratification is one of modifying the scattering triggered by non-shelf-similar topography, an appropriate functional form for the scattering coefficient is

$$
C_{\text {sc }}=W(h)[1+G(S)]
$$

Here, $W(h)$ represents the scattering induced by topographic variation alone; if the topography is shelf-similar $W(h)=0$ and $C_{s c}$ is zero regardless of the stratification. The function $G(S)$, where $S$ is some measure of the strength of the stratification, should be zero at zero stratification and increase with $S$ so that the factor $[1+G(S)]$ accounts for the amplification of the scattering observed in the numerical experiments.

In CTW theory (e.g. Huthnance, 1978; Chapman, 1983), the strength of the stratification is measured by the Burger number $S=N H / f L$, where $L$ is the width of the variable topography and $H$ is the depth of the deep ocean. A simple form for $G$ is found when $C_{o c}$ values calculated from the numerical results for non-shelf-similar topographies, normalized by the zero 


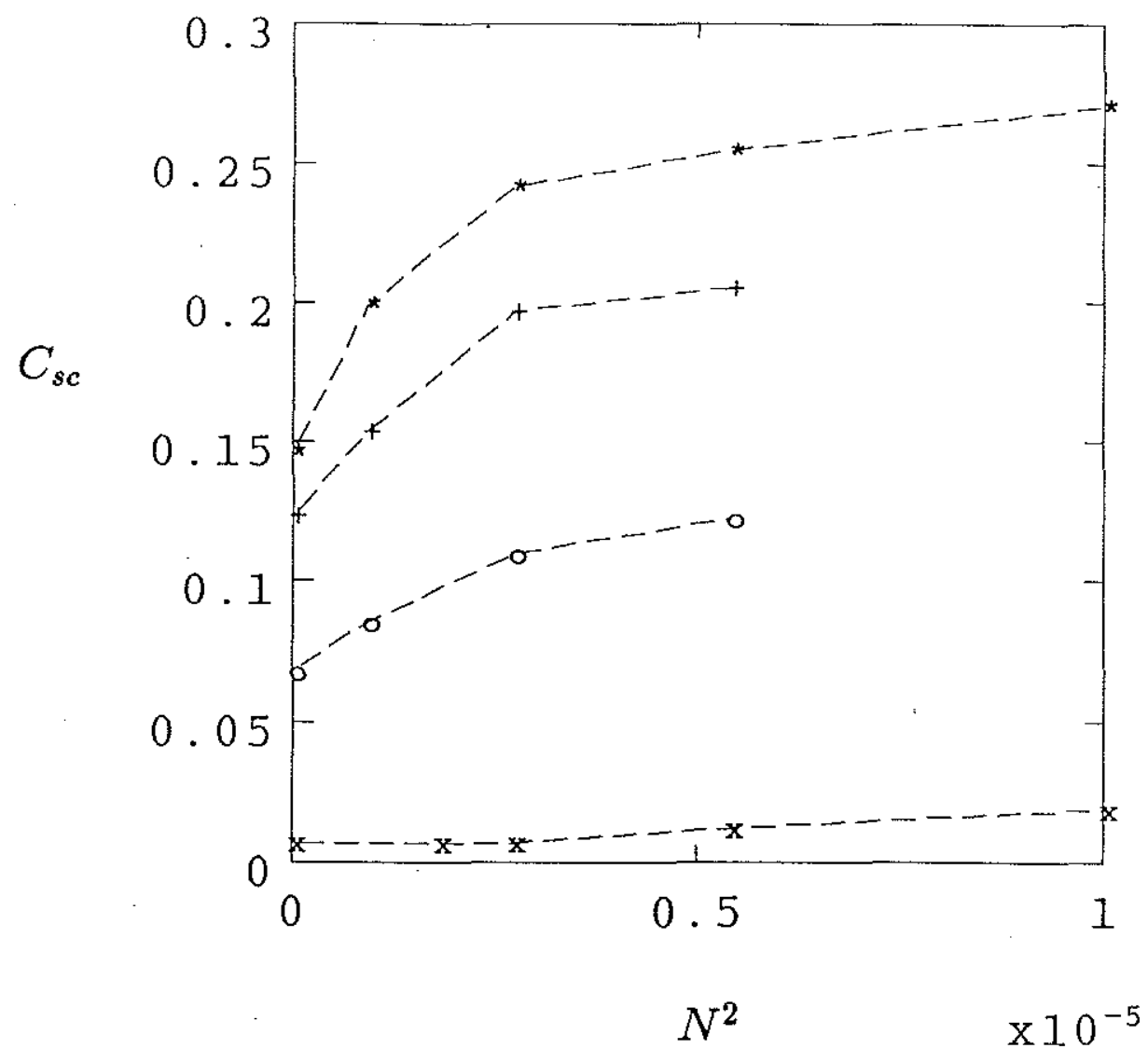

Figure 5-8: Scattering coefficient $C_{s c}$ defined in equation (5.2) vs. $N^{2}$ for the four sets of narrowingshelf scattering simulations. Symbols correspond to the topographies of: $x$, Figure 5-6; o, Figure 5-4; + , Figure 5-5; *, Figure 5-7.

stratification value for each topography, are plotted against $S$ (Figure 5-9). (This should be equivalent to plotting $C_{s c} / W(h)$ vs. $S$.) The simulation results for every topography fall close to a single straight line, indicating that for the moderate values of $S$ considered here, $G$ is an approximately linear function, say $G=\beta S$. The straight line passing through $G(0)=0$ which best fits the data in Figure 5-9 has slope $\beta=2.01$.

The function $W(h)$ measures the amount of scattering that occurs at zero stratification. From the works of Hsueh (1980) and Davis (1983) it is to be expected that $W(h)$ is related to the extent to which the topograpliy departs from shelf-similarity, and may therefore be thought of as being proportional to some topographic "warp" factor. An approximate form 


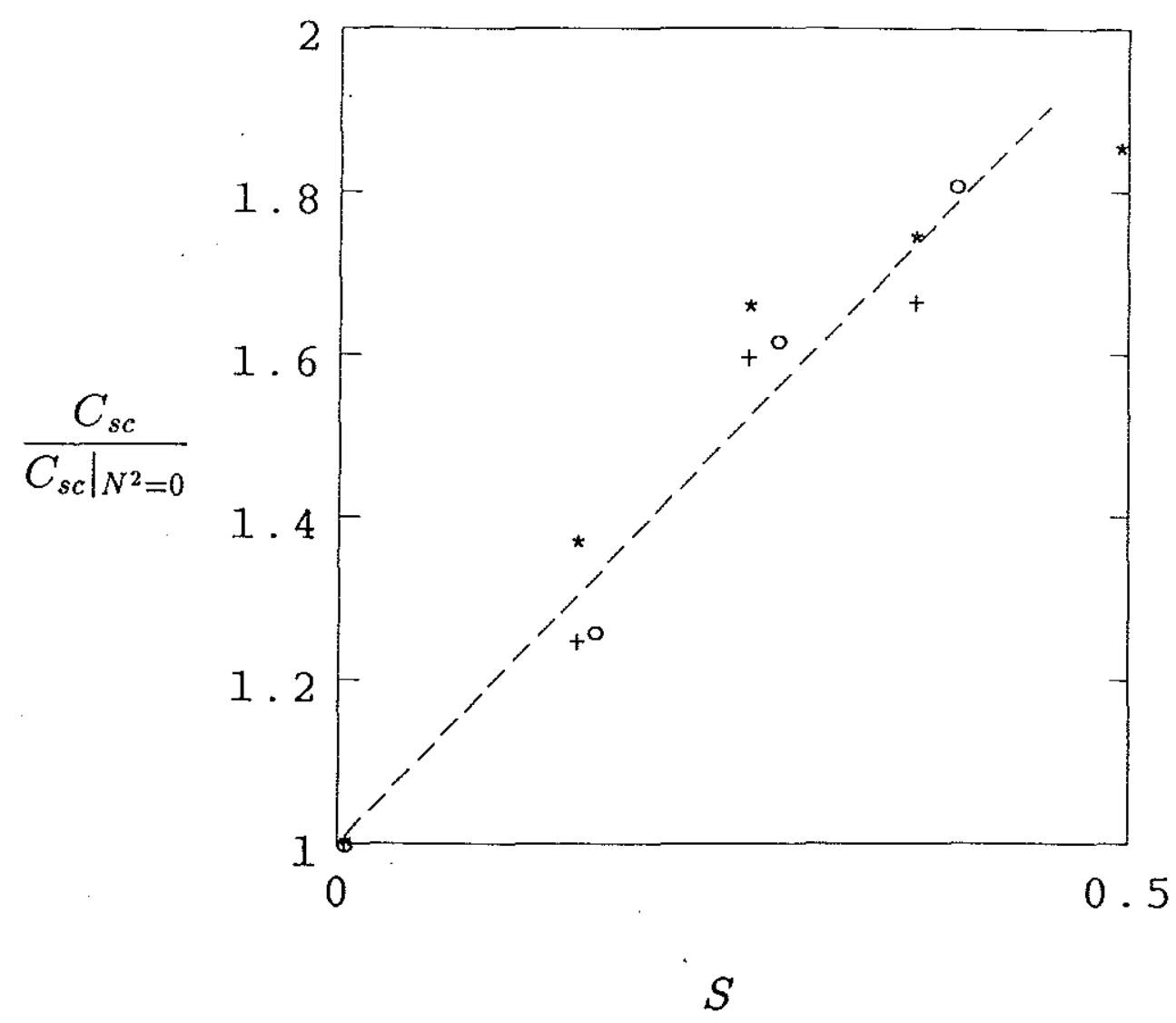

Figure 5-9: Scattering coefficient $C_{s c}$ values for the non-shelf-similar topographies, normalized by $\left.C_{s c}\right|_{N^{2}=0}$, vs. Burger number $S=N H / f L$. This is equivalent to plotting $C_{s c} / W(h)$ (see equation (5.2)), and shows that the stratification induced amplification of the scattering is an approximatley linear function of $S$, say $1+\beta S$. The dashed line fitted to the points has a slope of $\beta=2.01$. Symbols correspond to Figure 5-8.

for the warp factor can be obtained by examining Davis' (1983) equation (2.6), from which it is straightforward to show that a departure from shelf-similarity leaves a term on the right hand side of the equation for the streamfunction $(\psi)$ which is not eliminated by a change of coordinates. The term is proportional to the inner product of $\nabla \psi$ and $\nabla \times \mathbf{k} \frac{h}{h_{s, s}}$, where $\nabla$ is the gradient operator, $\mathbf{k}$ is a unit vertical vector, and $h_{s s}$ is the shape the topography would have if the depth profile at the entrance to the scattering region were carried through the width transition in a shelf-similar manner. Figure 5-10 illustrates the definition of $h_{s s}$. If the coastline is given by $y=c(x)$, and the location where the shelf reaches a uniform abyssal depth is given 


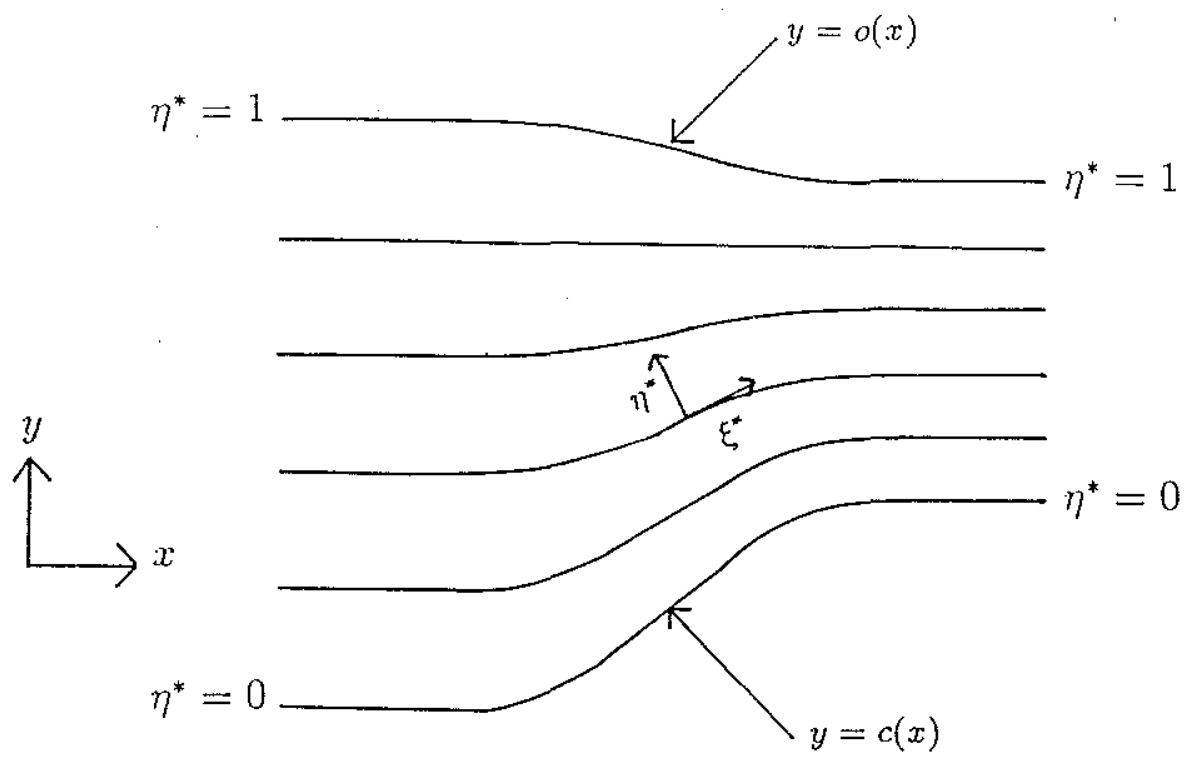

Figure 5-10: Coordinate system used in the definition of shelf warp factor.

by $y=o(x)$, then, according to Hsueh's definition, the isobaths of a shelf-similar topography follow lines of constant $\eta^{*}$ given by

$$
\eta^{*}=\frac{y-c(x)}{o(x)-c(x)}
$$

Therefore,

$$
h_{s s}\left(\eta^{*}(x, y)\right)=h\left(\eta^{*}(0, y)\right)
$$

Strictly speaking, $\nabla \psi$ cannot be determined without solving for the CTw flow-field. However, for the purposes of this discussion, and in order to determine an a priori estimate of the scattering induced by a given topography, the following proxy for $\nabla \psi$ is suggested. The vector $\nabla \psi$ is dominated by its across-shelf component which is simply the alongshelf transport, and since the alongshelf transport for a free barotropic shelf wave generally takes a maximum where the across-shelf slope is greatest, a suitable proxy for $(\nabla \psi)_{\text {across-shelf }}$ is $\frac{\partial h}{\partial s_{\eta^{*}}}$ where $s_{\eta^{*}}$ is a coordinate with dimensions of length in the direction of $\eta^{*}$. Having made this assumption, the magnitude of the term $\nabla \psi \cdot \nabla \times \mathbf{k} \frac{h}{h_{s s}}$ is approximated by

$$
\frac{\partial h}{\partial s_{\eta^{*}}} \frac{\partial}{\partial s_{\xi^{*}}}\left(\frac{h}{h_{s s}}\right)
$$

where $s_{\xi^{*}}$ is an 'across-shelf' coordinate perpendicular to $s_{\eta^{*}}$ (see Figure 5-10). Since $h_{s}$ is a function of $\eta^{*}$ only, $\frac{\partial}{\partial s_{\xi^{*}}}\left(\frac{1}{h_{s s}}\right)=0$, and the quantity in (5.6) can be integrated over the region 
of variable topography to give an estimate of the warp factor, $F_{w}$ :

$$
F_{w}=\int_{x} \int_{\eta^{*}=0}^{1} \frac{\partial h}{\partial s_{\eta^{*}}} \frac{1}{h_{s s}} \frac{\partial h}{\partial s_{\xi^{*}}} d \eta^{*} d x
$$

Note that this formulation satisfies the observed property that if the length of the region of topographic change doubles, the warp factor is unaltered because any decrease in $\frac{\partial h}{\partial \varepsilon_{\xi^{*}}}$ is compensated by an increase in the alongshelf length of the domain of integration.

Evaluating the warp factor $F_{w}$ for the four narrowing shelf topographies considered so far gives the values $0,1.71 \times 10^{-2}, 3.27 \times 10^{-2}$ and $3.99 \times 10^{-2}$. To examine how the function $W(h)$ depends on $F_{w}$, the values of $C_{s c} /(1+\beta S)$ for each topography are plotted against the corresponding warp factor in Figure 5-11. The points fall close to a single straight line suggesting that $W(h)$ is an approximately linear function of $F_{w}$, say $W(h)=\alpha F_{w}$. The straight line passing through the origin which best fits the data in Figure 5-11 has a slope of $\alpha=3.76$.

This discussion of the (rather arbitrarily defined) scattering coefficient, though somewhat speculative, has proven useful for synthesizing the results of the narrowing shelf scattering simulations. It is now apparent that topography and stratification act largely independently in the CTW scattering process. Stratification amplifies, by a simple linear gain, the scattering induced by topographic variations which in turn can be related to a topographic warp factor that estimates the degree to which the topography departs from shelf-similarity. A fairly simple empirical relationship for the scattering coefficient $C_{s c}$ has been derived:

$$
C_{s c}=\alpha F_{w}(1+\beta S)=3.76 F_{w}(1+2.01 S)
$$

In principle, the parameters $S$ and $F_{w}$ can be computed for any narrowing shelf geometry of interest and (5.8) used to predict the proportion of the energy flux of an incident mode 1 wave which will be scattered into higher modes. The success of the warp factor analysis is encouraging and suggests that a more rigorous examination of the effects of deviations from shelf-similarity may prove to be a fruitful direction for future analytical studies.

\subsection{Widening Shelf Results}

Two series of scattering simulations have been conducted to examine how topographic variations and stratification affect CTW scattering on widening shelves. The first series of simulations 


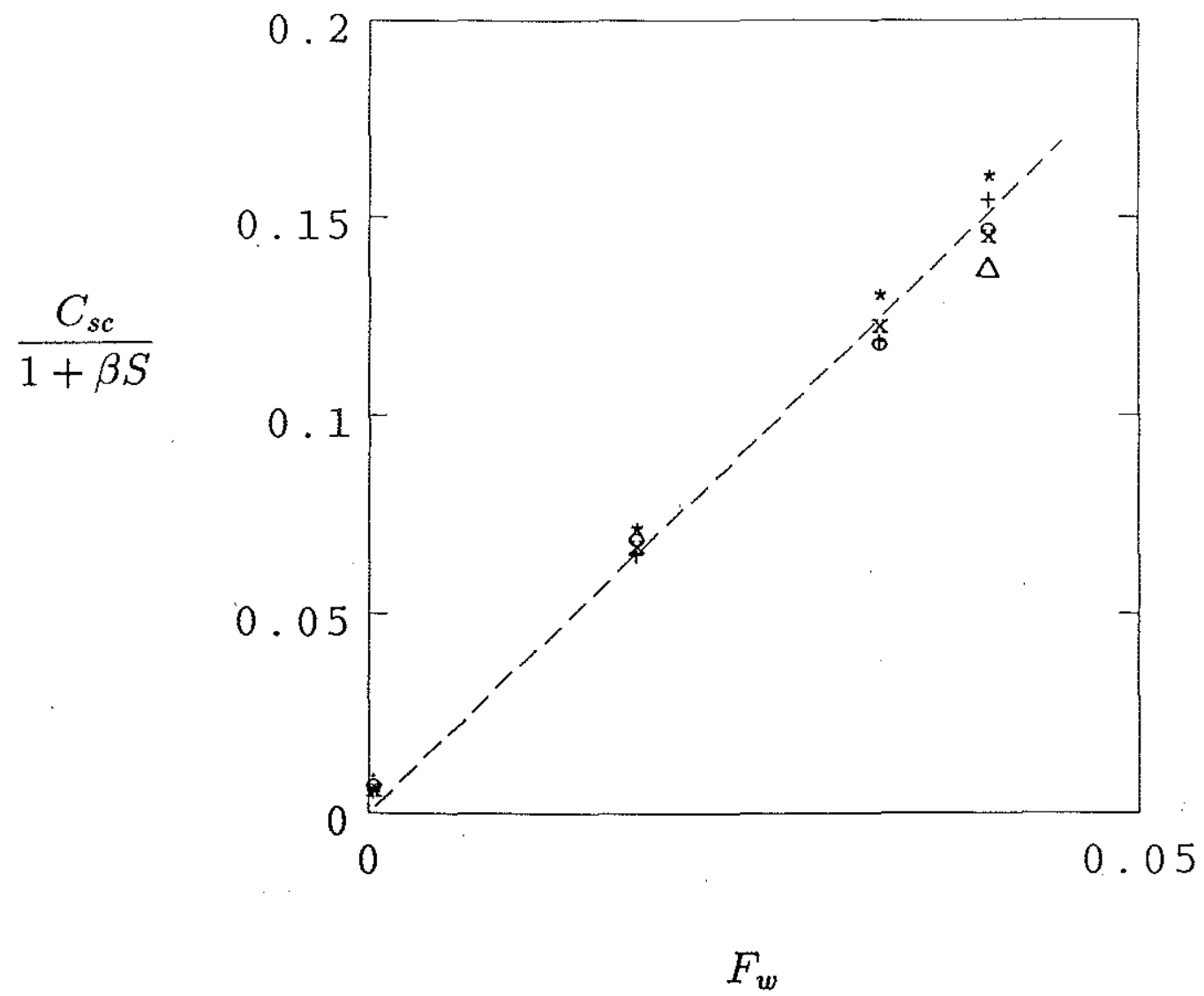

Figure 5-11: Scattering coefficient $C_{a c}$, normalized by $1+\beta S$, for the four narrowing shelf topographies vs. topographic warp factor $F_{w}$ computed from equation (5.7). This is equivalent to plotting the function $W(h)$ (see equation 5.3) and shows that $W(h)$ is an approximately linear function of $F_{w}$, say $\alpha F_{w}$. The dashed line fitted to the points has slope $\alpha=3.76$. Symbols correspond to different stratifications: $x$, $N^{2}=0 ;+, N^{2}=9 \times 10^{-7}$ (and $\left.1.8 \times 10^{-6}\right) ; *, 2.7 \times 10^{-6} ; \circ, 5.4 \times 10^{-6} ; \triangle, 1 \times 10^{-5}$.

examines the scattering of CTWs by the topography shown in Figure 5-12, which is simply a reversal of the narrowing shelf topography shown in Figure 5-5. The energy fluxes of the transmitted modes, for an incident mode 1 wave with frequency $1 \times 10^{-5} \mathrm{~s}^{-1}$, are presented in Table 5-5. Comparison with Table 5-2 shows that the scattering induced by the widening topography of Figure 5-12 is similar in strength to that induced by the "reciprocal" narrowing topography. In this example, the shelf width increases by a factor of approximately 1.6. In the analytical study of Chapter 3, a relative width change of this value scattered approximately $40 \%$ of the energy flux of a mode 1 incident wave into higher modes (Figure 3-3). The geometry 


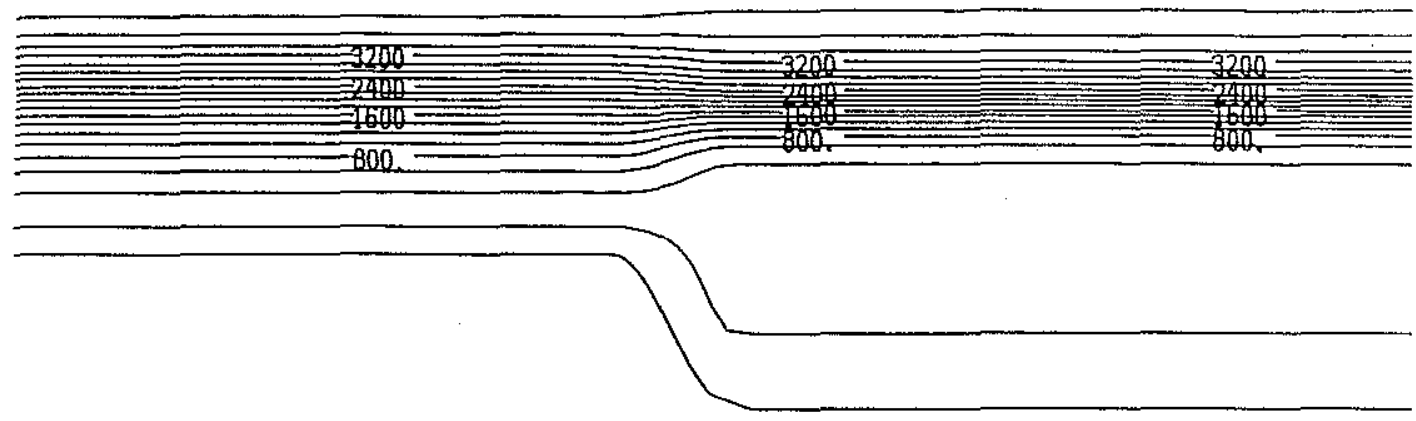

Figure 5-12: Bathymetry used in the first series of widening shelf scattering experiments. Contour interval is $200 \mathrm{~m}$. The depth profiles outside the scattering region are those of Figure 5-1.

of the problem considered in Chapter 3 (Figure 3-2) includes a substantial change in the depth at the coast. Given the tendency of low-frequency flow to follow isobaths, this depth change alone is likely to contribute significantly to the strength of the scattering. It is to be expected then, that the more realistic topographic variation of the present numerical experiment would be somewhat less conducive to Crw scattering.

With the exception of the modal energy fluxes for the barotropic case, the results in Table 55 exhibit the same trend observed in the narrowing shelf experiments; scattering is amplified by stratification. In Figure 5-13, the scattering coefficient $C_{s c}$ for each of the runs in Table 5-5, normalized by the zero stratification value, is plotted against Burger number $(S)$. Except for the zero stratification result, the points fall close to a line with the same slope as the function $1+\beta S$ (shown by the dashed line) fitted to the narrowing shelf results (Figure 5-9). The departure of the barotropic result from the simple functional form for the scattering coefficient postulated in equation (5.3) is possibly due to the fact that in the barotropic limit transmitted mode 3 is very close to being evanescent at this frequency. The results presented in Chapter 3 show that local minima in the energy flux of transmitted mode 1 occur near parameter values at which 


\begin{tabular}{cccccc}
\hline \multirow{5}{*}{ Mode } & \multicolumn{5}{c}{$N^{2}\left(\mathrm{~s}^{-2}\right)$} \\
& 0 & $9 \times 10^{-7}$ & $2.7 \times 10^{-6}$ & $5.4 \times 10^{-6}$ & $1 \times 10^{-5}$ \\
& & & & \\
\hline & & & & \\
1 & 0.848 & 0.858 & 0.831 & 0.791 & 0.744 \\
2 & 0.121 & 0.137 & 0.164 & 0.197 & 0.240 \\
3 & 0.030 & $2.1 \times 10^{-3}$ & $1.0 \times 10^{-3}$ & $8.7 \times 10^{-3}$ & 0.010 \\
4 & - & $30 \times 10^{-3}$ & $4.7 \times 10^{-3}$ & $3.4 \times 10^{-3}$ & $6.5 \times 10^{-3}$ \\
& & & & & \\
& $(1.040)$ & $(1.041)$ & $(1.048)$ & $(1.056)$ & $(0.949)$ \\
\hline
\end{tabular}

Table 5-5: Energy fluxes of the transmitted modes generated when a mode 1 incident wave with frequency $1 \times 10^{-5} \mathrm{~s}^{-1}$ encounters the variable topography of Figure 5-12 for different values of the stratification $\left(N^{2}\right)$. Table entries are calculated in the same manner as Table 5-1.

higher transmitted modes become evanescent (Figures 3-5 and 3-6). It is likely then, that the low value of the energy flux in transmitted mode 1 for this topography and zero stratification, results from the anomalously high value obtained for the nearly evanescent mode 3 .

The second series of simulations considers CTW scattering at a fixed stratification of $N^{2}=$ $5.4 \times 10^{-6} s^{-2}$ for several different widening shelf topographies; namely, those obtained when the topographies of Figures 5-4 through 5-7 are reversed. The energy fluxes of the scattered modes are presented in Table 5-6 with the columns arranged left to right in order of increasing departure from shelf-similarity. The same trend observed in the narrowing shelf experiments is evident; scattering strength increases with increasing departure from shelf-similarity.

Comparison of the values in Table 5-6 with the corresponding entries in Tables 5-1 through 54 shows that, overall, the strength of the scattering for each widening topography is quite similar to that observed for its reciprocal narrowing topography. For example, the transmitted mode 1 energy fluxes for the narrowing shelf topographies for $N^{2}=5.4 \times 10^{-6} s^{-2}$ are, in order of increasing non-shelf-similarity: $0.988,0.877,0.795$ and 0.746 , which are close to the reciprocal widening shelf values in row 1 of Table 5-6.

The topographic warp factors $F_{w}$ for these widening topographies are $0,7.81 \times 10^{-3}, 7.85 \times$ $10^{-3}$ and $9.82 \times 10^{-3}$. In a plot analogous to Figure 5-11 (for narrowing shelves), Figure 5-14 


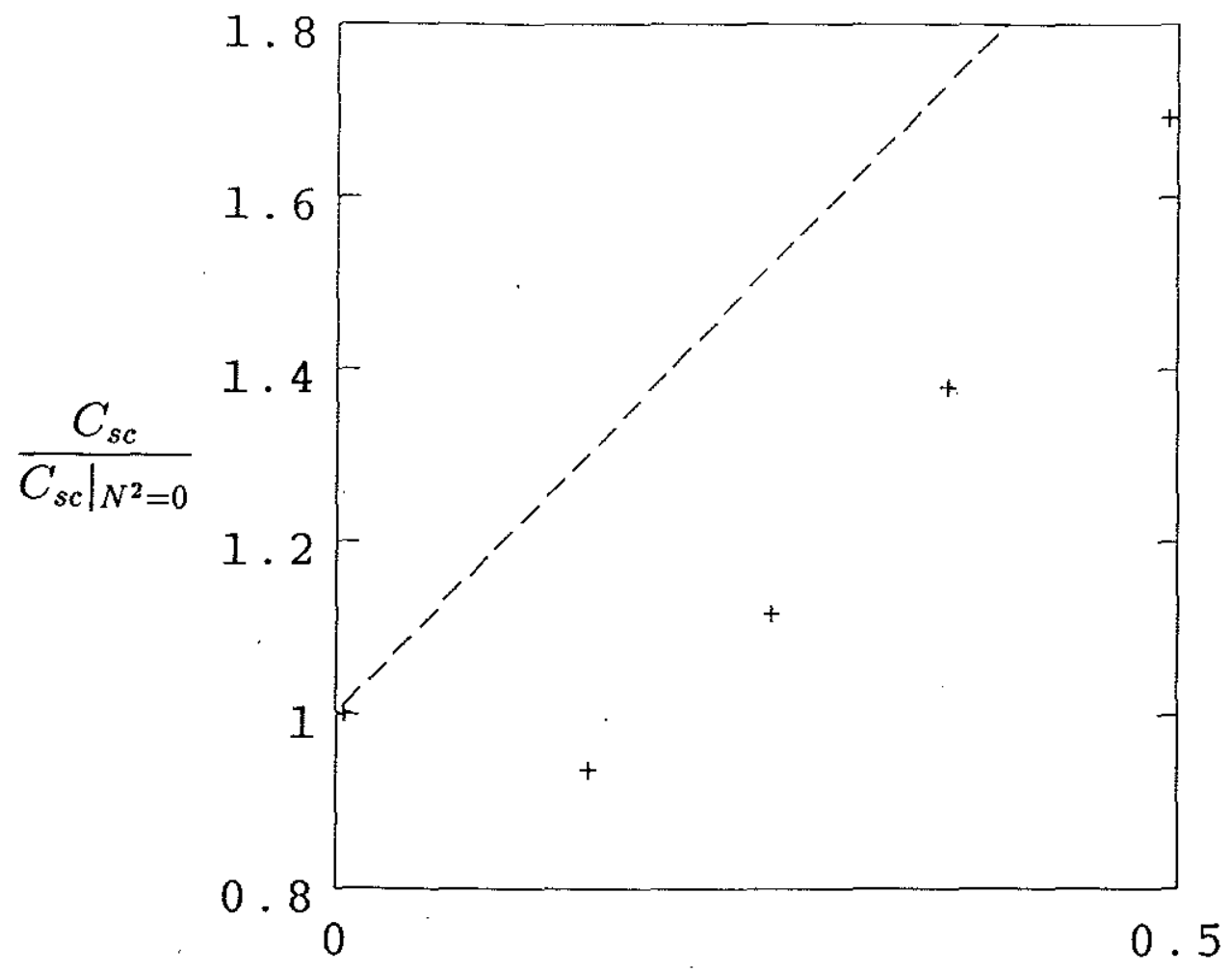

$S$

Figure 5-13: Scattering coefficient $C_{s c}$ values for the topography of Figure 5-12, normalized by $\left.C_{s c}\right|_{N^{2}=0}$, vs. Burger number $S=N H / f L$. The dashed line is the function $1+\beta S$ fitted to the narrowing shelf results (Figure 5-9).

shows the scattering coefficients $C_{s c}$ for this series of runs, normalized by $1+\beta S$, plotted against $F_{w}$. In the normalization $\beta=2.01$ was used since the results of the last series of experiments suggest that the value of $\beta$ determined from the narrowing shelf experiments is valid for widening shelves also. The points plotted do not cluster as closely to a single straight line as they did for the narrowing shelf scattering simulations. Futhermore, the slope of a straight line fitted to these widening shelf results differs by a factor of roughly 3.5 from the slope of the line fitted to the narrowing shelf results. Therefore, while it is clear that scattering strength is related to the simple definition of warp factor proposed in equation 5.7, some refinements to the definition are required before it can be applied reliably to the prediction of the strength of CTw scattering 


\begin{tabular}{ccccc}
\hline \multirow{2}{*}{ Mode } & \multicolumn{5}{c}{ Topography } \\
\cline { 2 - 5 } & \multicolumn{5}{c}{ A } & B & C & D \\
\cline { 2 - 5 } & & & & \\
\hline 1 & 0.979 & 0.867 & 0.791 & 0.757 \\
2 & 0.011 & 0.132 & 0.197 & 0.238 \\
3 & $7.0 \times 10^{-3}$ & $1.2 \times 10^{-3}$ & $8.7 \times 10^{-3}$ & $4.1 \times 10^{-3}$ \\
4 & $2.6 \times 10^{-3}$ & $2.7 \times 10^{-4}$ & $3.4 \times 10^{-3}$ & $1.1 \times 10^{-3}$ \\
& $(1.099)$ & $(1.098)$ & $(1.056)$ & $(1.044)$ \\
& & & & \\
\hline
\end{tabular}

Table 5-6: Energy fluxes of the transmitted modes generated when a mode 1 incident wave with frequency $1 \times 10^{-5} s^{-1}$ encounters different widening shelf geometries; namely, those obtained by reversing the topographies of: Figure 5-6 (column A), Figure 5-4 (column B), Figure 5-5 (column C), and Figure 5-7 (column D). The columns are arranged left to right in order of increasing departure from shelf-similarity. The topographic warp factors for these topographies are $0,7.81 \times 10^{-3}, 7.85 \times 10^{-3}$ and $9.82 \times 10^{-3}$ for A through D respectively. For all cases the stratification is $N^{2}=5.4 \times 10^{-6} \mathrm{~s}^{-2}$.

on continental shelves of practical interest.

\subsection{Flow Field Near the Scattering Region}

Discussion thus far has concentrated on the gross scattering effects of irregular coastal geometry; namely, the amplitudes of the transmitted modes which propagate freely away from an isolated scattering region. A comparison of narrowing and widening topographies showed that the amplitudes of the freely propagating transmitted modes generated by "reciprocal" narrowing and widening topographies are quite similar. However, significant differences are found in the scattering effects of narrowing and widening geometries when the flow fields close to the topographic variation are examined.

\section{Widening Shelves}

Barotropic shelf waves propagating through the widening shelf geometry considered in the analytical study of Chapter 3 (Figure 3-1) were found to leave a shadow zone of weak flow behind the coastline bend. This occurs because low-frequency flow has a tendency to follow $f / h$ contours (isobaths on an $f$-plane) and therefore requires a finite alongshelf distance to adjust 


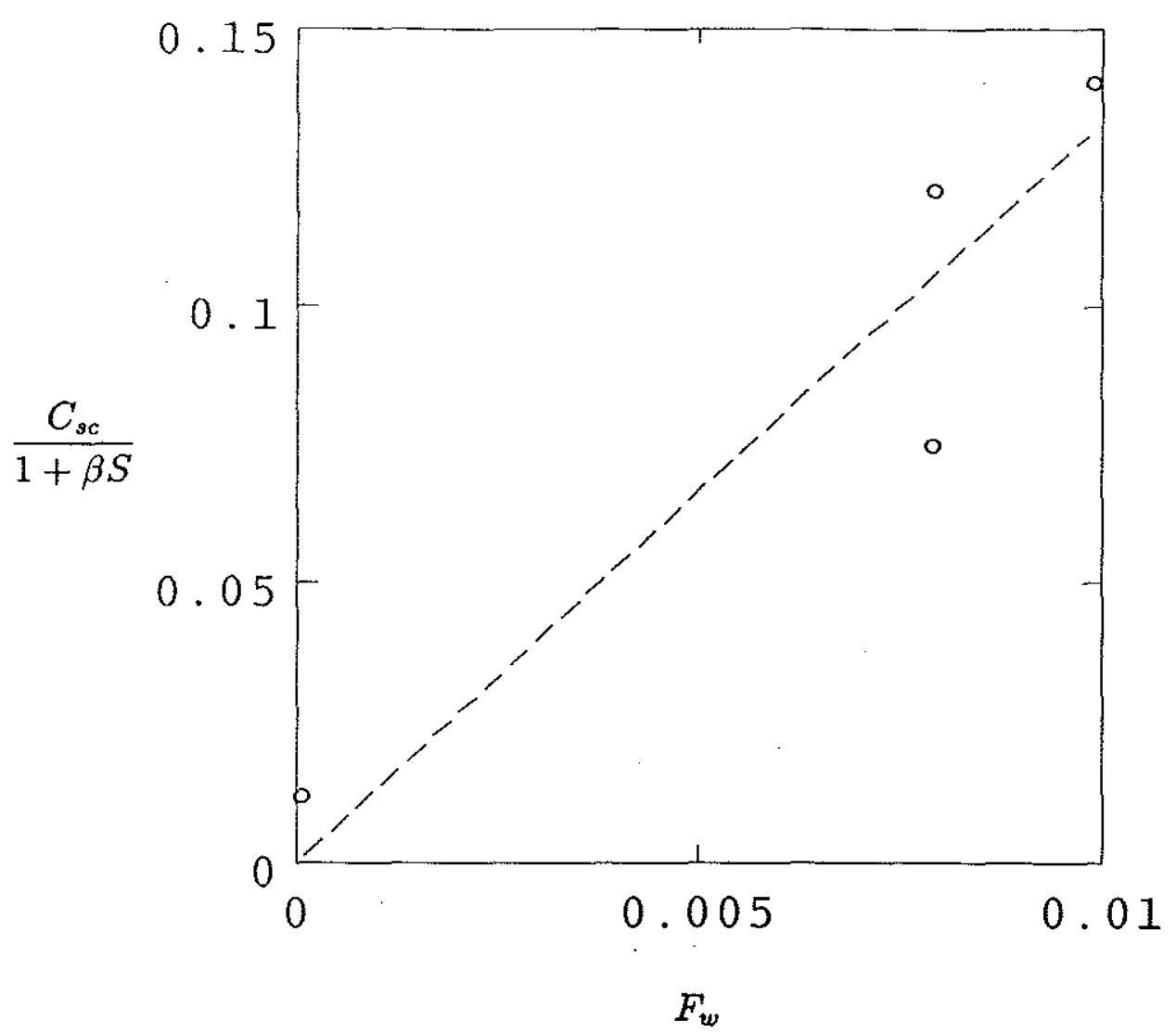

Figure 5-14: Scattering coefficient $C_{s c}$, normalized by $1+\beta S$, for the widening shelf results of Table 5-6 vs. topographic warp factor $F_{w}$ computed from equation (5.7). The value of $\beta$ used is that determined from the, narrowing shelf experiments (Figure 5-9). The dashed line fitted to the points has a slope of 13.3 .

to the changing depth at the coast. More realistic idealizations of irregular shelf topography, such as those considered in the numerical experiments, have a depth at the coast which does not vary appreciably alongshelf, and is small relative to the deep ocean depth. Questions arise, then, as to whether or not a shadow zone would occur on more realistic shelves, and whether, in fact, the strong scattering found in the analytical study is a consequence primarily of the unrealistically severe depth change at the coastal wall.

The latter question has already been answered in the preceeding two sections. It was shown that for the more realistic topographies considered in the numerical experiments, the strength of the scattering is somewhat less than that observed for comparable relative width changes 
in the analytical problem. Nevertheless, it was evident that a significant proportion of CTW energy is still scattered into modes other than that of the incident wave. The former question, concerning the existence of a shadow zone, can be addressed by examining the scattered wave flow field in the vicinity of the topographic and coastline variation, for some of the widening shelf model runs.

Figure 5-15 shows a sequence of plots of surface velocity vectors at different times during one wave period, for the case of a mode 1 incident Crw with frequency $1 \times 10^{-5} \mathrm{~s}^{-1}$ propagating through the topography of Figure $5-12$, for $N^{2}=5.4 \times 10^{-6} s^{-2}$. The transmitted wave energies for this case are shown in the fourth column of Table 5-5. Note that the across-shelf scale in Figure 5-15 has been expanded for clarity, and that only the vectors at every third alongshelf grid point and every second across-shelf grid point of the numerical model are plotted. The topography is also shown (with the correct aspect ratio of horizontal scales). Figure 5-16 shows the sequence of plots of the locally alongshelf component of the surface velocity corresponding to Figure 5-15, along with a plot of the coordinate grid used in the simulation (with the correct aspect ratio of horizontal scales). The 'aiongshelf' velocity component is in the direction of the $\xi$ computational coordinate.

In the first frame of Figures 5-15 and 5-16, a crest of the mode 1 incident wave is approaching from the left. In the next two frames the crest reaches the region of varying topography and the subsequent scattering produces considerable variation in the velocity field over short spatial scales. A cell of intense alongshelf flow trapped closely to the inflection in the coastline is particularly evident in frame 3. The fourth frame corresponds to the time at which the amplitude of transmitted mode 1 is almost zero at the location where the mode fit is performed (indicated by the single tick marks). Consequently, the flow there is dominated by the signature of transmitted mode 2 , i.e. there are two zero crossings in the across-shelf structure of the alongshelf velocity. In frame 5 an intense coastal return flow occurs in the scattering region as the trough of the incident wave reaches the coastline bend.

A more detailed picture of the time variation of the coastal currents is shown in Figure 5-17, where the alongshelf velocity at the coast is plotted as a function of alongshelf coordinate $\xi$, and time $t$, for the entire duration of the simulation. Each line across the field of view corresponds to a snapshot of the coastal alongshelf velocity. A section through the plot, into the background, 

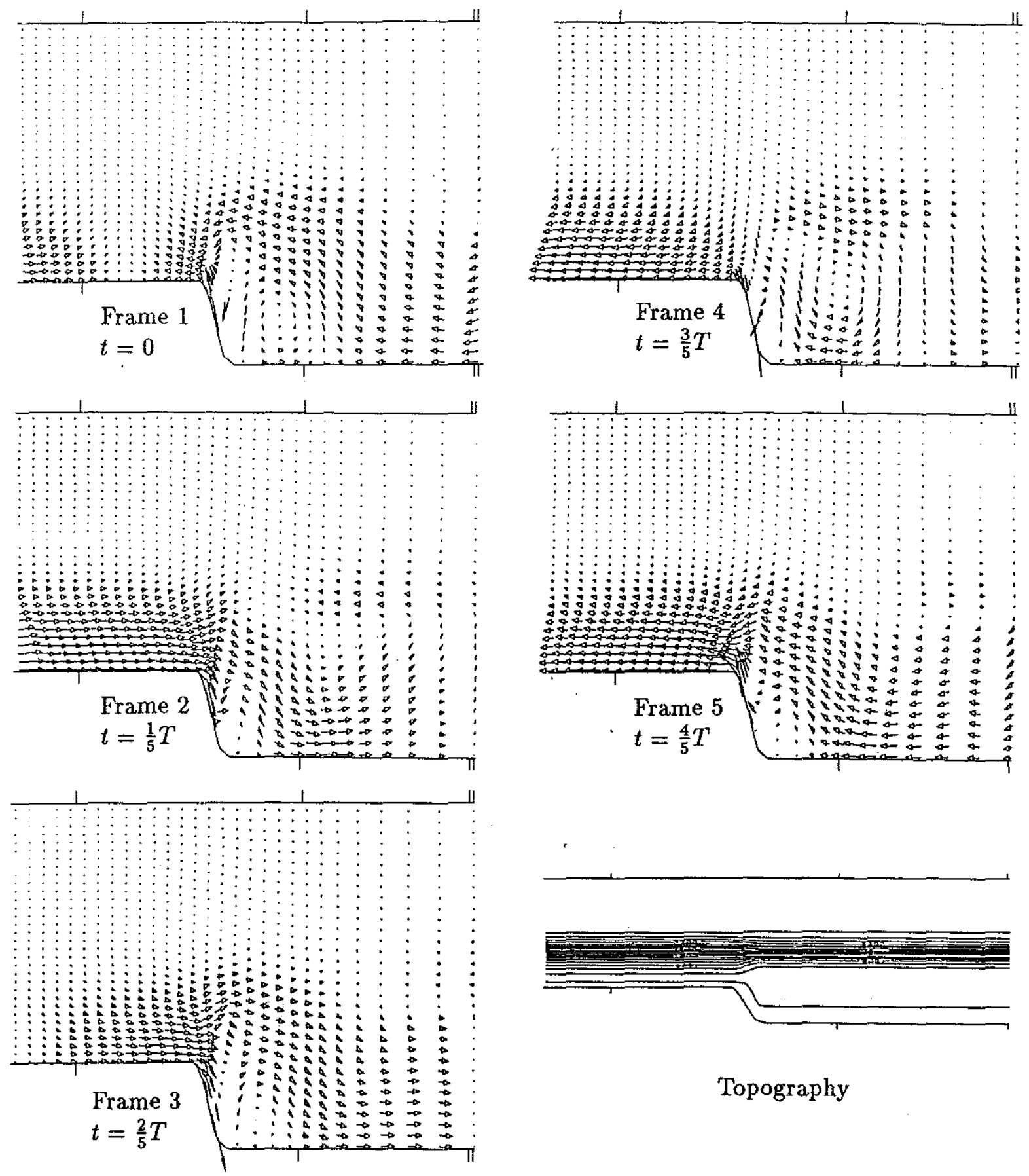

Topography

Figure 5-15: A sequence of plots of surface velocity vectors at different times $t$ during a single wave period $(T)$. The case shown is a mode 1 incident CTw with frequency $1 \times 10^{-5} s^{-1}$ propagating through the topography of Figure 5-12. $N^{2}=5.4 \times 10^{-6} \mathrm{~s}^{-2}$. The transmitted energy fluxes for this case are shown in column 4 of Table 5-5. Note that the across-shelf scale has been expanded for clarity and that only the vectors for every third alongshelf and every second across-shelf grid point of the numerical model are shown. The topography is shown with the correct aspect ratio of alongshelf and across-shelf scales. 

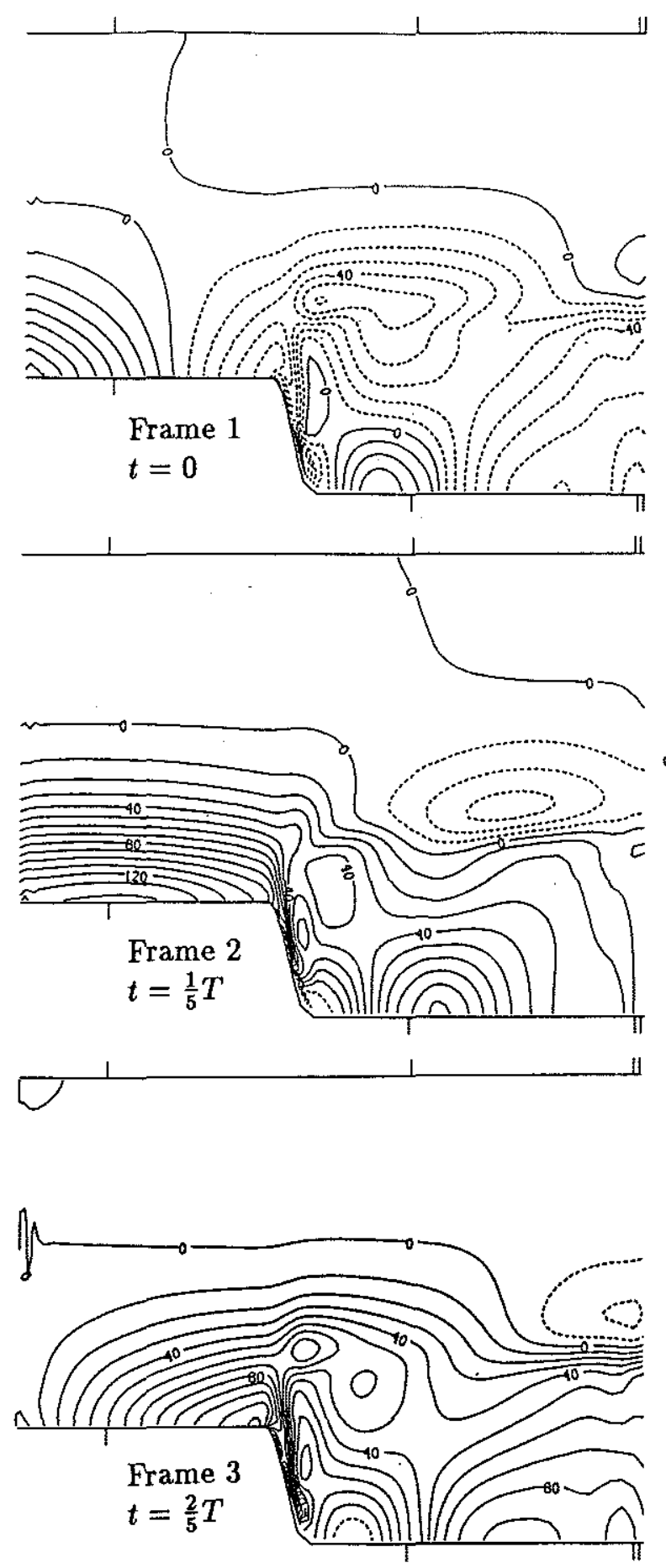
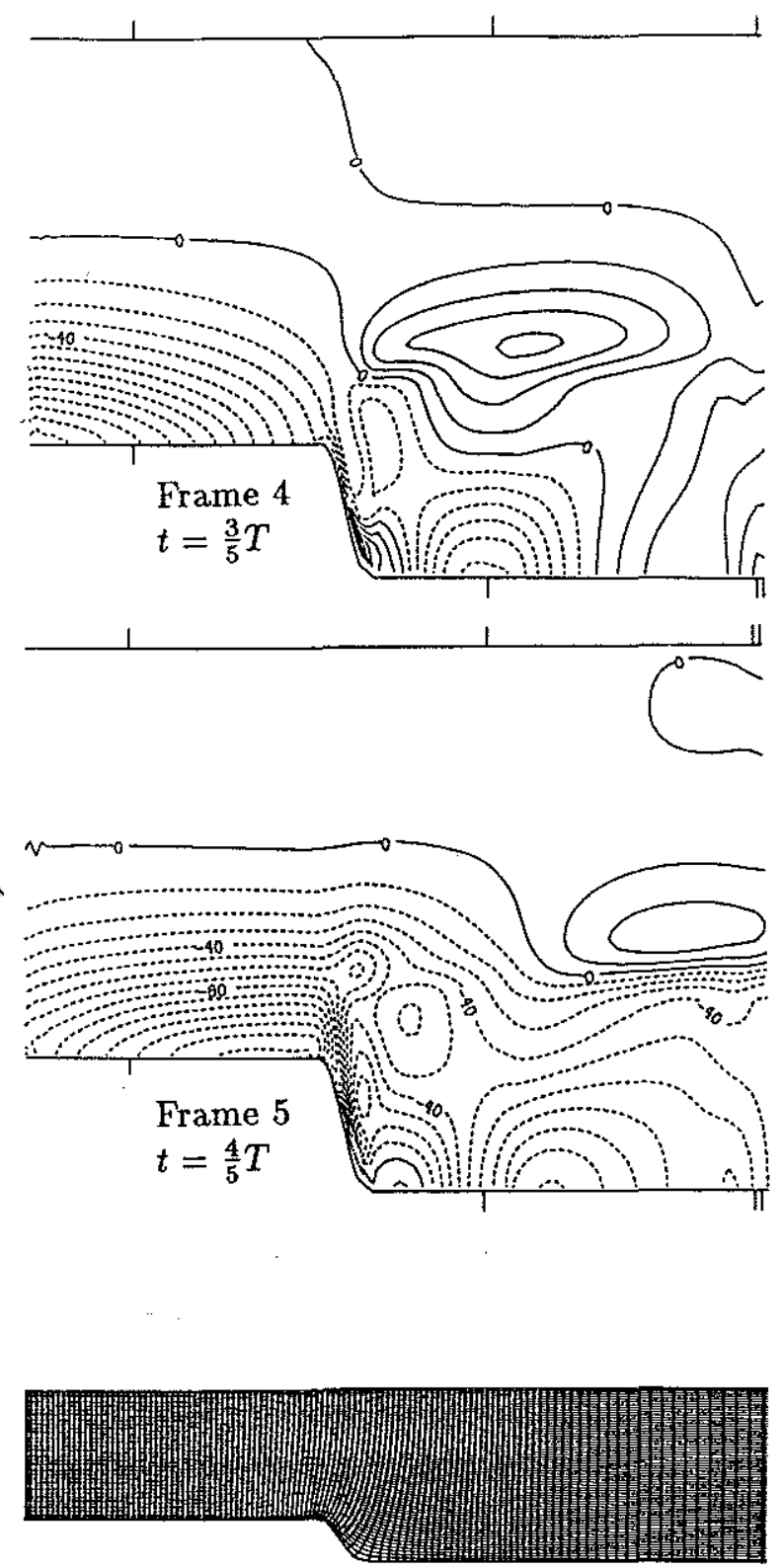

Coordinate Grid

Figure 5-16: A sequence of plots of the surface alongshelf velocity (i.e. velocity in the direction of the $\xi$ computational coordinate) for the same case shown in Figure 5-15. The coordinate grid used in the simulation is shown with the correct aspect ratio. 


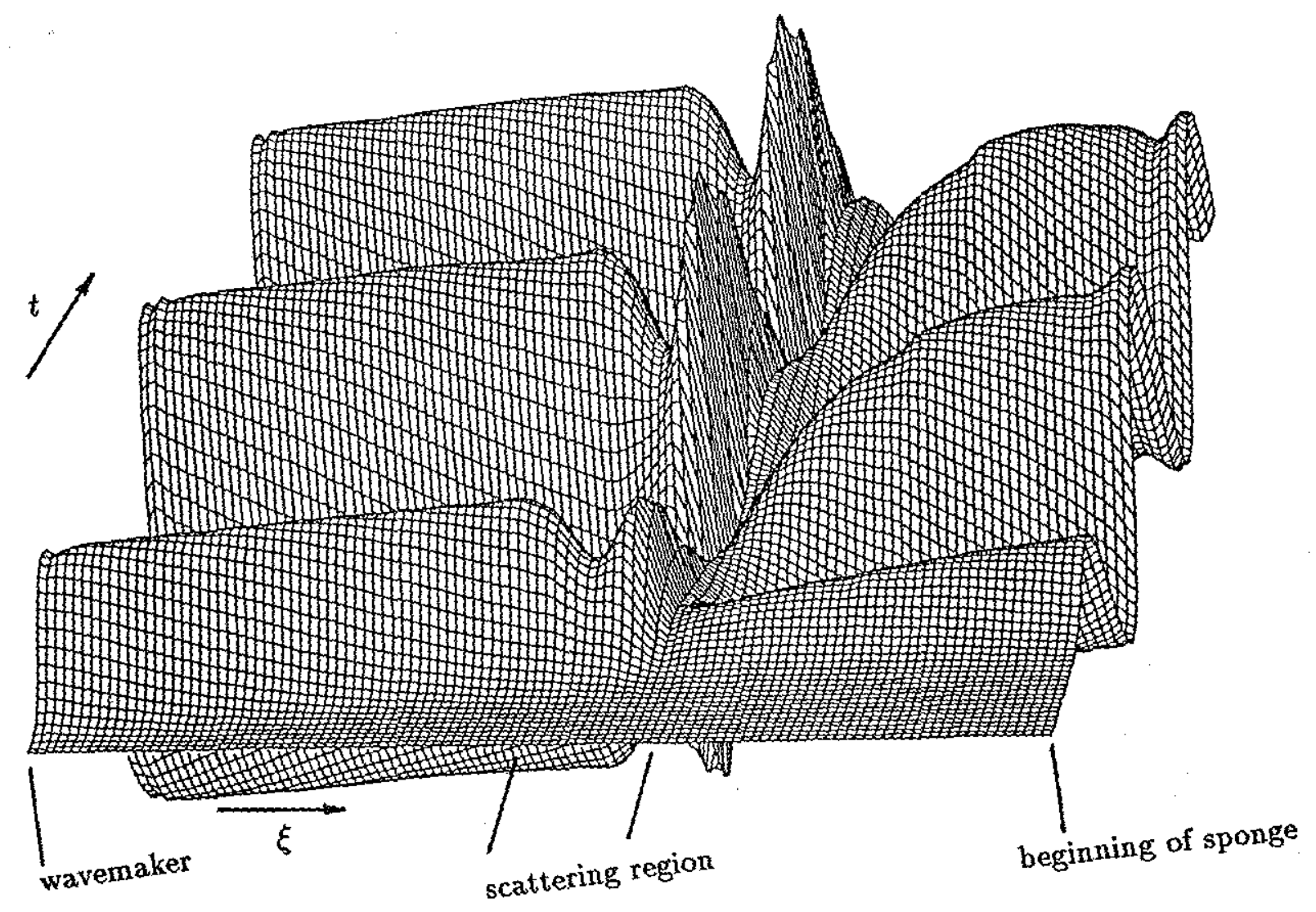

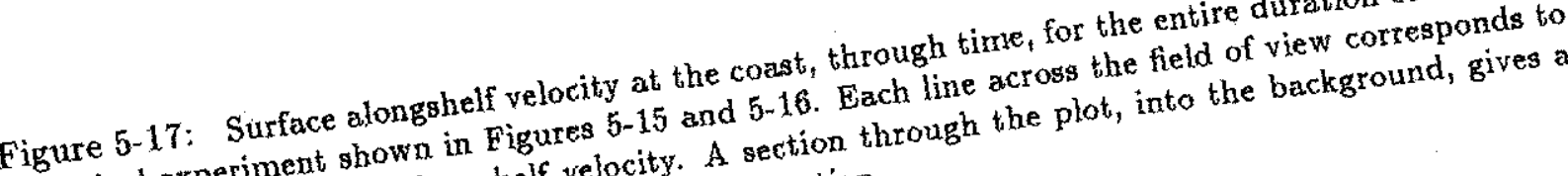
numerical experiment show in Elonghelf velocity. A section throu.

a snapshot of the coastal at a particular coastal location.

time series of the velocity at a particu 
gives a time series of the velocity at a particular coastal location.

At the beginning of the simulation (i.e. the foreground of the plot) the numerical wavetank is quiescent. As time advances (into the background) the amplitude of the wavemaker increases over one wave period, and then oscillates with fixed amplitude for two additional wave periods. Following the first crest of the wavetrain through the coastline bend, the wave-induced current first diminishes, then increases abruptly, and then drops to almost zero before the scattered waves emerge from the scattering region. This pattern is fully established once the second wave crest has passed through the scattering region. The sequence of peaks in Figure 5-17 empliasizes the intense current events associated with the passage of each wave, while the saddles indicate the zones of weak flow.

This pattern is considerably more complex than that observed in the analytical scattering study (Chapter 3). While there is a region of weak coastal flow immediately downstream from the coastline bend, this is not the signature of a shadow zone. The existence of a shadow zone would be indicated by weak currents throughout the scattering region, whereas here it is found that within the scattering region intense coastal currents occur which exceed in strength those of the incident wave. Farther downstream the pattern is qualitatively similar to the analytical results - the interaction of multiple transmitted waves with different wavelengths modulates the flow field. Note that it takes two to three wave periods for the downstream flow field to become truly periodic. This is due to the slower propagation speeds of transmitted modes 2 and 3. The straight ridges of constant height 'upstream' from the scattering region in Figure 5-17 show that there is no reflection.

In Figure 5-18, a comparison is made of these wave-induced coastal currents to those generated by the other widening shelf geometries considered in section 5.3. The figure shows the maximum alongshelf velocity at each location along the coast, normalized by the maximum coastal velocity of the incident wave. The curves are labelled $\mathrm{A}$ through $\mathrm{D}$ to correspond with the columns of Table 5-6. Curve $\mathrm{C}$ is therefore the "envelope" of the oscillatory currents depicted in Figure 5-17. The origin of the alongshelf coordinate is the beginning of the scattering region, which is $100 \mathrm{~km}$ long for cases A, C and D, and $200 \mathrm{~km}$ long for case $\mathrm{B}$.

Cases $B$ and D exhibit shadow zones qualitatively similar to that obtained in the analytical study. In both cases the width of the continental shelf increases rapidly in the scattering region 


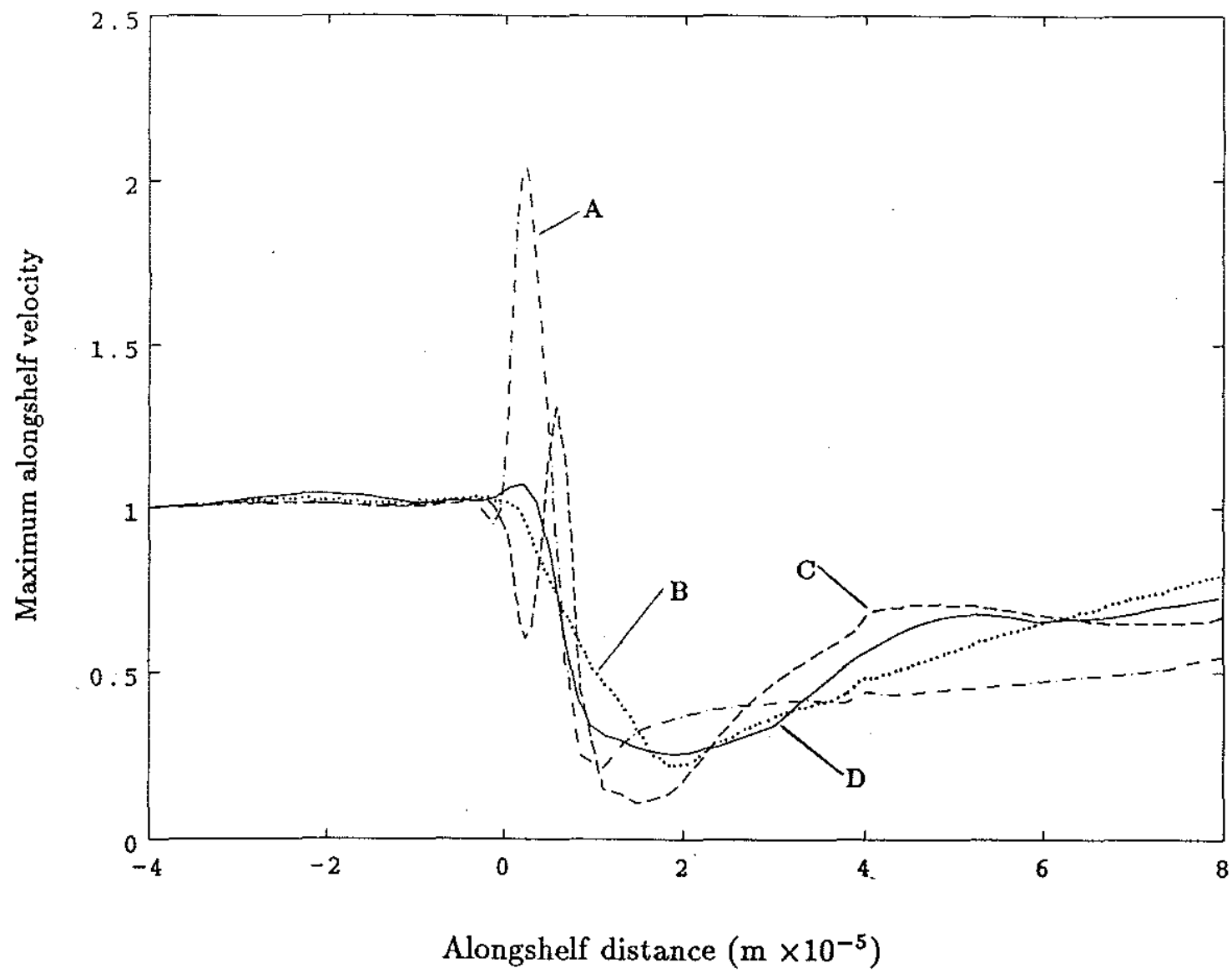

Figure 5-18: Maximum alongshelf velocity at the coast for the four widening shelf geometries considered in Table 5-6. Labels A through D correspond to the columns of Table 5-6.

and it is tempting to invoke the 'flow-following-isobaths' mechanism as an explanation for the presence of a shadow zone. However, the shelf depth profiles outside the scattering region in case $\mathrm{D}$ are identical to those in case $\mathrm{C}-$ the difference in the two topographies being limited to the shape of the coast. The markedly different response within the scattering region for these two cases demonstrates that the scattering process active here is more complex than that proposed to explain the analytical results.

Of particular interest is case $\mathrm{A}$ - the shelf-similar widening topography. For this topography the incident mode 1 wave emerges from the scattering region without any appreciable loss of energy (Table 5-6). It might have been expected, then, that the flow would exhibit a smooth transition from the across-shelf structure of mode 1 on the incident side to that of mode 1 
on the transmitted side. In actuality, there is a strong intensification of the coastal velocity immediately following the start of the coastline bend, and an equally significant decrease in the coastal flow shortly thereafter. Since in this case there is no reflection, and the amplitudes of the scattered waves are very small, the strong spatial modulation of the flow within the region of variable topography must be due to non-propagating (i.e. evanescent) flow structures excited within the scattering region.

\section{Narrowing Shelves}

Examination of the narrowing shelf model runs provides further evidence that evanescent wave-like motions contribute significantly to the flow field induced by CTWs near regions of variable topography. In a plot similar to Figure 5-15 for the widening shelf example, Figure 519 shows a sequence of plots of surface velocity vectors at different times during a wave period, for the case of a mode 1 incident CTw encountering the topography of Figure 5-5, for $N^{2}=$ $5.4 \times 10^{-6} s^{-2}$. The transmitted wave energies for this case are shown in column 4 of Table 5-2. As in the widening shelf example, there are regions within the area of changing topography where the wave-induced currents are amplified significantly. It is also evident, particularly in frames 3 and 5 , that the directions of the wave-induced currents vary considerably over short spatial scales, indicating the possibility of rapid phase changes.

A feature not observed in the widening shelf example is a noticeable upstream influence by the scattered wave field on the incident-wave flow. The across-shelf component of the currents associated with the long incident wave is weak, and as a result the velocity vectors in the region well to the left of the scattering region in each frame of Figure 5-19 are aligned almost exactly alongshelf. However, near to the scattering region, perturbations to this solely alongshelf flow are evident (frames 1,3 and 4). This indicates that the scattering has introduced an appreciable across-shelf component to the CTW flow field near to, yet still upstream from, an abrupt narrowing of the continental shelf. This occurs despite the stratification being sufficiently strong to eliminate any possibility of propagating reflected CTws (i.e. $\omega<\omega_{\infty}$,wide $)$.

The alongshelf structure of this upstream influence can be seen in Figure 5-20, which shows the alongshelf velocity at the coast, through time, in the same manner as Figure 5-17. Immediately upstream from the coastline bend the coastal currents exhibit characteristics of an evanescent wave mode; namely, the envelope of the current fluctuations has a sinusoidal 

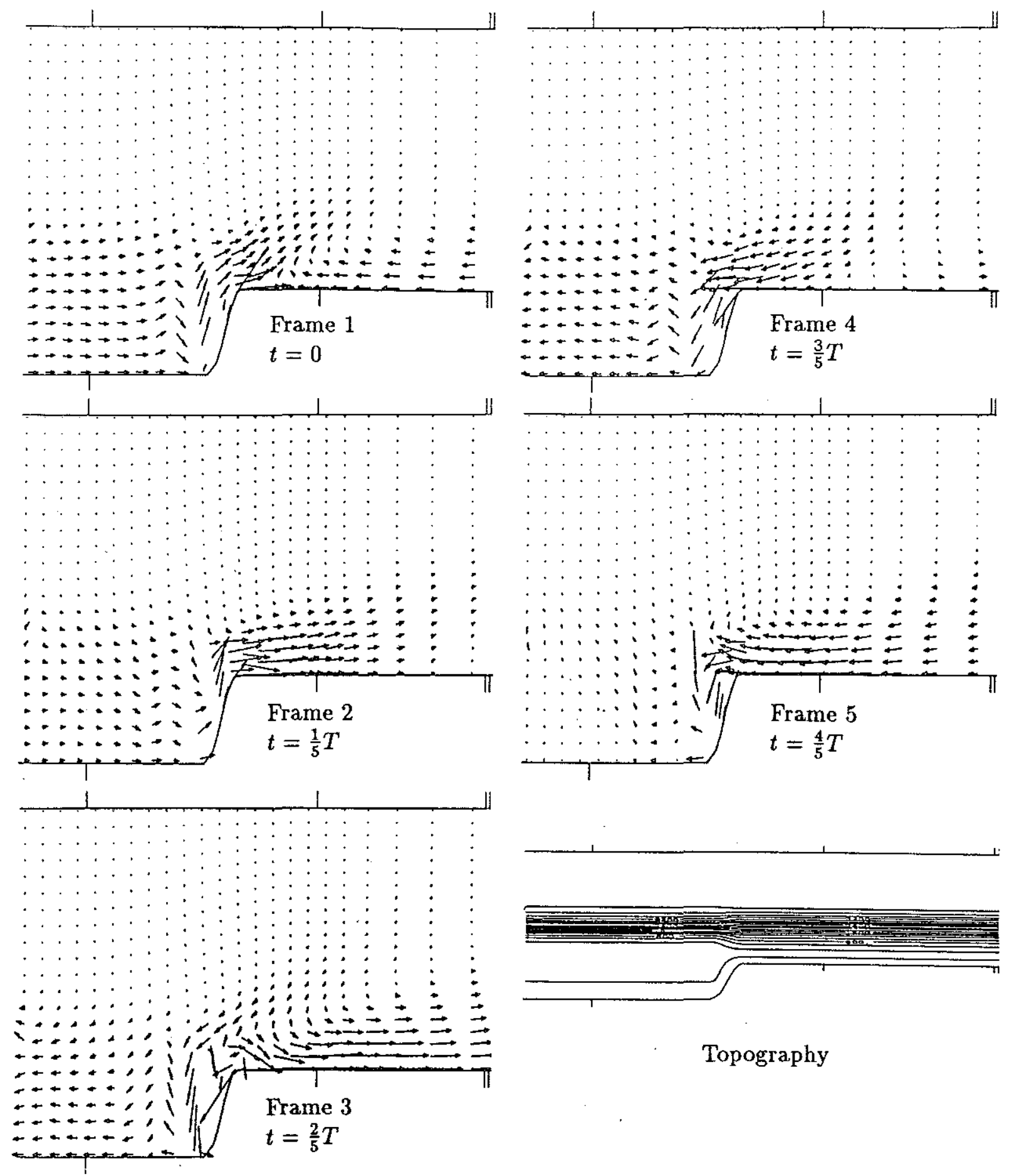

\section{Topography}

Figure 5-19: A sequence of plots of the surface velocity vectors at different times during a single wave period. The case shown is a mode 1 incident CTW with frequency $1 \times 10^{-5} \mathrm{~s}^{-1}$ propagating through the topography of Figure 5-5. $N^{2}=5.4 \times 10^{-6} s^{-2}$. The transmitted energy fluxes for this case are shown in column 4 of Table 5-2. Note that the across-shelf scale has been expanded for clarity. The topography is shown with the correct aspect ratio. 


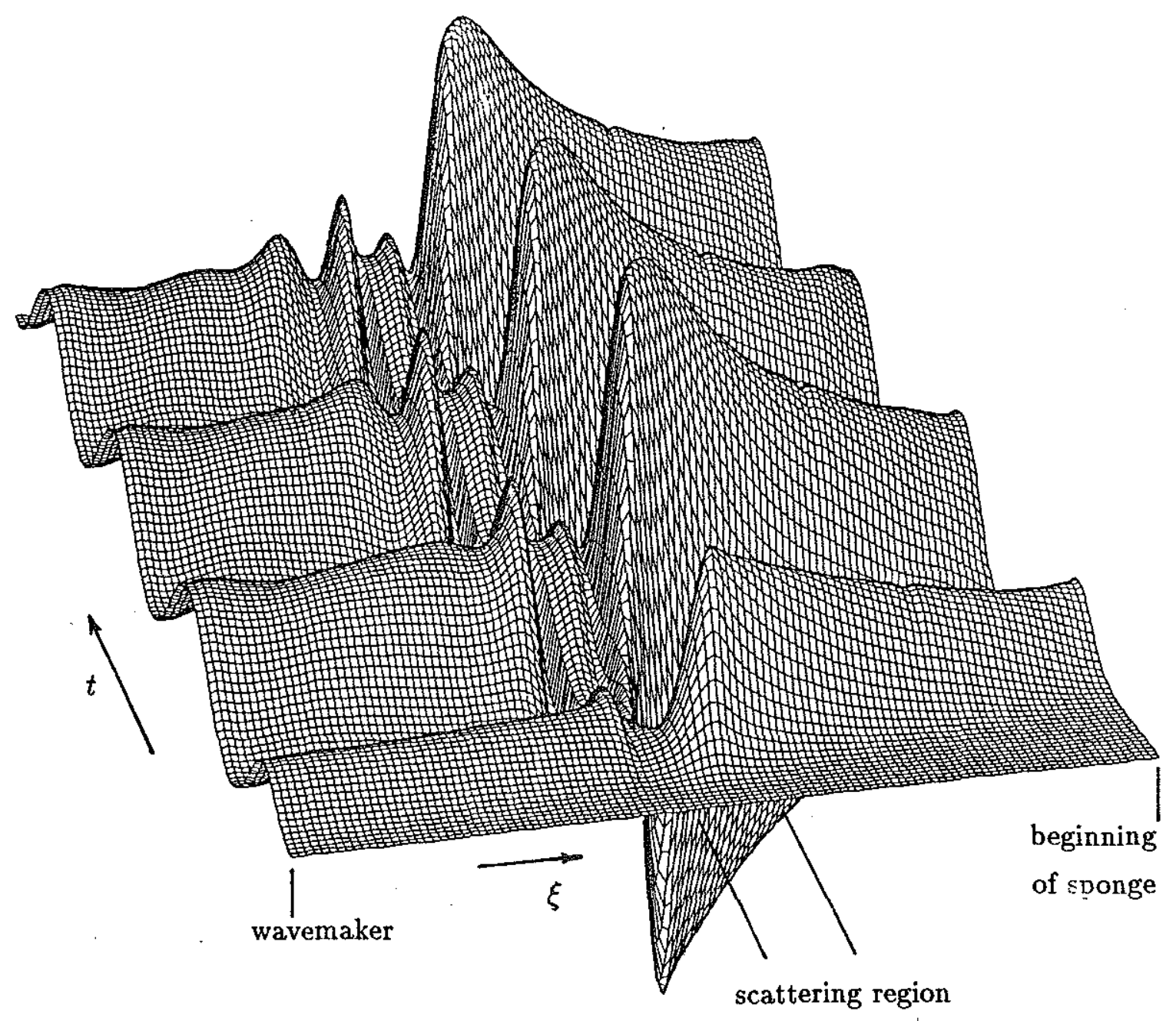

Figure 5-20: Surface alongshelf velocity at the coast, through time, for the entire duration of the same numerical experiment shown in Figure 5-19. Each line across the field of view corresponds to a snapshot of the coastal alongshelf velocity. A section through the plot, into the background; gives a time series of the velocity at a particular coastal location. 


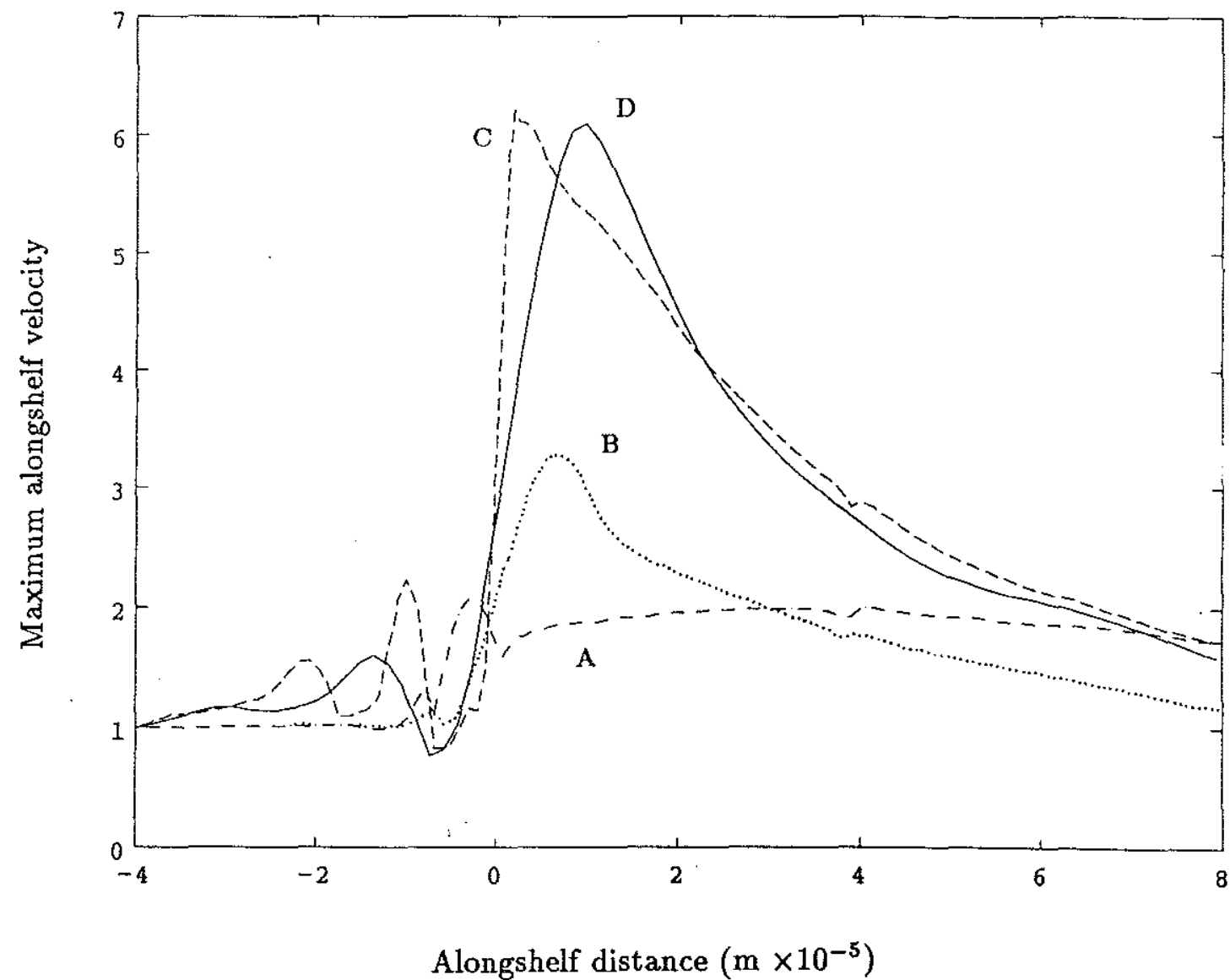

Figure 5-21: Maximum alongshelf velocity at the coast for the four narrowing shelf geometries considered in section 5.2. Labels correspond to the $N^{2}=5.4 \times 10^{-6} s^{-2}$ runs of the following topographies: A, Figure 5-6; B, Figure 5-4; C, Figure 5-5; D, Figure 5-7.

alongshelf structure which decays exponentially away from the scattering region. The wavelength and alongshelf decay scale of this evanescent mode can be estimated from curve $\mathrm{c}$ of Figure 5-21, which shows the maximum alongshelf velocity at the coast. The mode decays over roughly $200 \mathrm{~km}$, and the two local maxima within this region indicate a wavelength of roughly $100 \mathrm{~km}$.

Figure 5-21 includes the maximum alongshelf velocities for the other three narrowing shelf topographies studied, for $N^{2}=5.4 \times 10^{-6} s^{-2}$. Cases $\mathrm{A}, \mathrm{B}$ and $\mathrm{D}$ correspond to the topographies of Figures 5-6, 5-4 and 5-7, respectively. The other cases exhibit patterns qualitatively similar to case $\mathrm{C}$. In all cases there is evidence of an evanescent mode influencing the flow field 
upstream from the scattering region. Furthermore, in all cases except A (shelf-similar), the coastal currents near the inflection in the coast exceed those outside the scattering region. In case $A$, the maximum coastal currents actually occur some $50 \mathrm{~km}$ upstream from the scattering region.

\section{A Comment on the Possible Role of Non-Linearities}

In all the simulation runs conducted in this study, the nonlinear terms of the governing equations have been omitted from the numerical computations. The justification for the neglect of these terms, when studying CTWs, is founded on a scale analysis of the full nonlinear primitive equations. It is straightforward to show that the nonlinear terms of the horizontal momentum equations (4.1), i.e. $(\mathbf{u} \cdot \nabla) \mathbf{u}+w \mathbf{u}_{z}$, are smaller than the smallest terms retained, $\mathbf{u}_{t}$, by a factor of $U / \omega L_{x}$. Here, $L_{x}$ and $U$ are characteristic alongshelf length and velocity scales, and $\omega$ is the wave frequency. For typical Crw scales $\left(U=10 \mathrm{~cm} \mathrm{~s}^{-1}, L_{x}=500 \mathrm{~km}\right.$, and $\left.\omega=10^{-5} \mathrm{~s}^{-1}\right)$, $U / \omega L_{x}$ is of the order $2 \times 10^{-2}$, and it would appear that the linear approximation is well founded. However, in the discussion of the near field flow it was shown that within a CTW scattering region there is generally a marked intensification of the wave-induced velocities, and the currents exhibit substantial variation over short spatial scales. Both of these effects increase the magnitude of the nonlinear terms in the momentum equations. This raises the question: is the linear approximation still valid within the scattering region?

To address this question thoroughly would require a series of numerical wavetank experiments, with the nonlinear terms of the governing equations included, designed to quantify (i) the dispersion of cTws in a straight channel caused by nonlinearities alone, and (ii) the modifications, if any, to the CTW scattering processes studied in the linear numerical wavetank experiments. In order to make the latter comparison, it would be necessary to assume that the nonlinear solutions can be decomposed into a set of freely-propagating linear Crw modes with the role of nonlinearity viewed as a modification to the coupling of these modes. This assumption is akin to that used in analytical 'weakly-nonlinear' wave studies.

Such a comprehensive investigation of nonlinear CTW scattering processes is beyond the scope of this study. However, an estimate of whether or not nonlinear processes might influence CTW scattering can be made by computing the magnitude of the omitted nonlinear terms based on the linear solutions already obtained. 
Consider the narrowing shelf scattering example discussed above. In frame 2 of Figure 5-19, the strength and direction of the wave-induced currents vary considerably within the scattering region. The terms on the left-hand-side of (4.1), at the time corresponding to frame 2 of Figure 5-19, are plotted in Figure 5-22. In this example the incident wave has a maximum alongshelf velocity at the coast of $10 \mathrm{~cm} \mathrm{~s}^{-1}$. For clarity, only the area in the neighborhood of the coastline bend is shown. In the alongshelf $(u)$ momentum equation (left-hand panels of Figure 5-22), the nonlinear terms should be compared to the acceleration term $\left(u_{t}\right)$, since the magnitude of $u_{t}$ is roughly half that of the Coriolis term $(-f v)$. Throughout most of the scattering region the nonlinear terms are smaller than the acceleration terms by a factor of approximately 10. However, in a small region roughly $40 \mathrm{~km}$ in length near the location of maximum convex curvature of the coastline, the nonlinearities are indeed comparable in strength to the acceleration. A similar result is obtained upon examination of the terms of the across-shelf $(v)$ momentum equation (right-hand panels of Figure 5-22). For the $v$ equation, the nonlinearities actually slightly exceed the acceleration term $\left(v_{t}\right)$ in the same narrow zone where the coastline curvature is greatest. In this region however, the Coriolis term $(f u)$ dwarfs both the acceleration and nonlinear terms by a factor of approximately 40 . It should be noted that in the longwave approximation, which is frequently invoked in the study of CTWs, it is the term $v_{t}$ that is neglected.

From this analysis it appears that, at least over a limited alongshelf distance within the scattering region, the possibility that nonlinear processes may have some effect on the dynamics of CTW scattering cannot be ruled out. The nature and strength of any nonlinear influence, especially given the limited extent of the reigon over which nonlinear terms appear to be strong, remains an open question.

\subsection{Summary}

The numerical experiments have shown that regions of rapidly changing coastline and topography cause appreciable scattering of CTW energy. The strength of the scattering, as measured by the proportion of the incident energy flux scattered into modes other than that of the incident wave, is proportional to a topographic warp factor which estimates the extent to which the topography departs from shelf-similarity. The scattering induced by non-shelf-similar topo- 

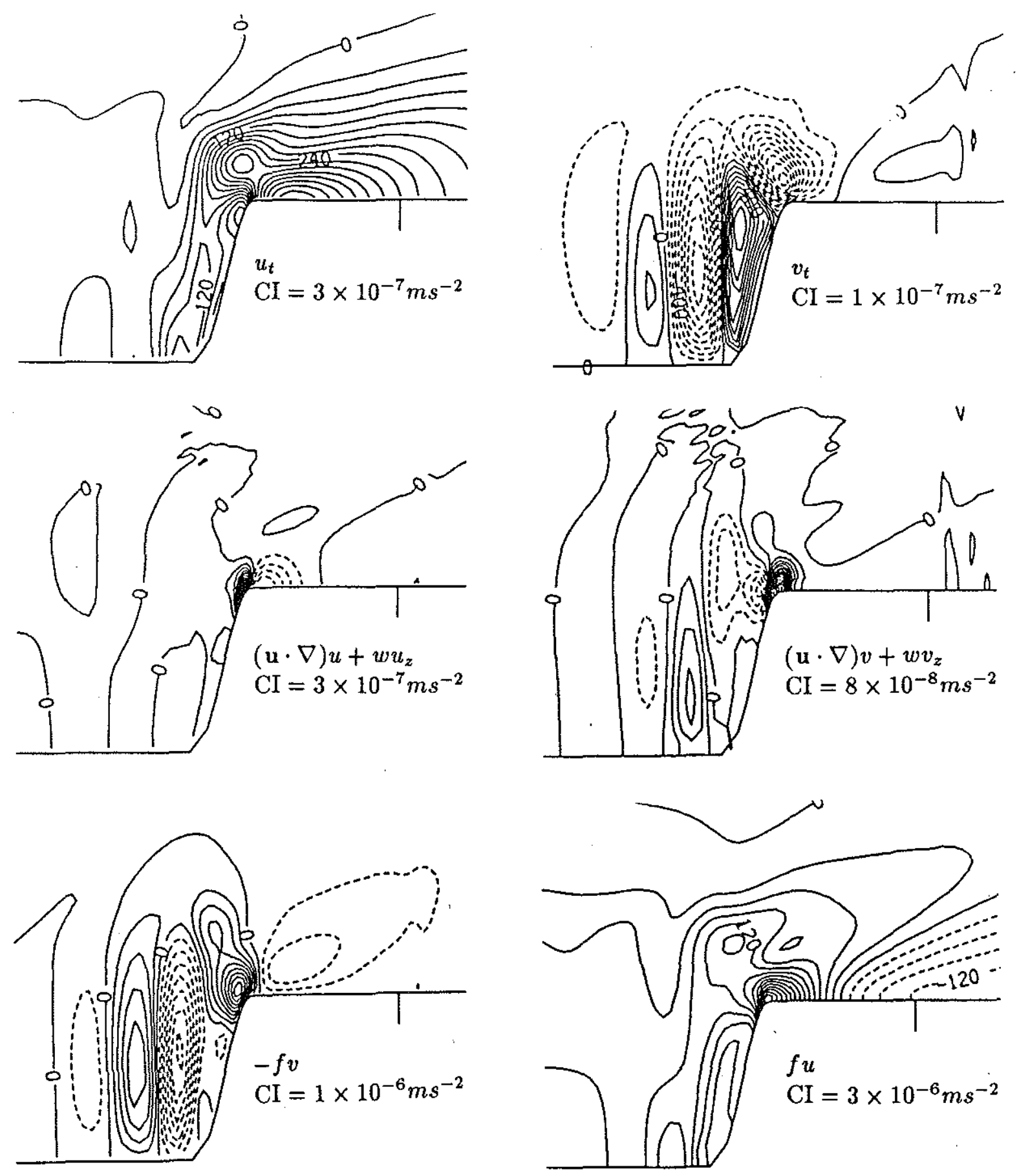

Figure 5-22: A comparison of the strength of the linear and nonlinear terms of the momentum equations within a CTW scattering region. The case shown corresponds to frame 2 of Figure 5-19. The incident wave has a maximum alongshelf velocity at the coast of $10 \mathrm{~cm} \mathrm{~s}^{-1}$. Left: alongshelf $(u)$ equation. Right: across-shelf $(v)$ equation. Note that the region shown is an enlargement of area in the neighborhood of the coastline bend. CI denotes the contour interval of each plot. 
graphic variations is amplified by density stratification. A comparison of the effects of widening and narrowing topographies showed that the amplitudes of the freely propagating transmitted modes generated by "reciprocal" narrowing and widening topographies are similar. Within the scattering region itself, the strengths of the scattered-wave-induced currents exhibit substantial variation over short spatial scales. On both widening and narrowing shelves, there is generally a marked intensification of the flow within the scattering region, and significant differences in the directions of the currents at points separated by a few tens of kilometers indicate the occurence of rapid variations in phase. On narrowing shelves, the influence of the scattering can extend upstream into the region of uniform topography.

In the next Chapter, the improved understanding of CTW scattering processes gained from this study is applied to the interpretation of a simulation of CTW scattering at a site along the East Coast of Australia where observations by Griffin and Middleton (1986) suggest the presence of scattered freely-propagating CTWs. 


\section{Chapter 6}

\section{Observations of Coastal-Trapped Wave Scattering: A Case Study}

The results of the studies described in Chapters 3 and 5 show that large irregularities in coastline and topography cause appreciable scattering of CTW energy into modes other than that of the incident wave. The generation of multiple transmitted modes with differing across-shelf structures and propagation speeds produces an alongshelf modulation of the wave-induced flow field which may be important observationally. Also of likely significance to observational studies is the intensification, and increase in spatial variability, of the wave-induced currents within the scattering region of many of the irregular coastal geometries considered in the numerical experiments (Chapter 5).

It was noted earlier (Chapter 3) that some qualitative features of the solution to the analytical scattering problem have been seen in the observations made by Griffin and Middleton (1986), hereafter referred to as GM, on the East Coast of Australia. In this Chapter, the study by GM is discussed further in light of the results of Chapter 5 , and by examining the results of a numerical wavetank simulation of the scattering of a CTW pulse by coastal geometry which approximates closely the geography of the GM study site.

GM made observations of sea level, current, and temperature at three across-shelf sections between Fraser Island (FI) and the southern end of the Great Barrier Reef (Figure 6-1). Their field program was designed to test a theory of the wind-driving of Bsws downstream from 


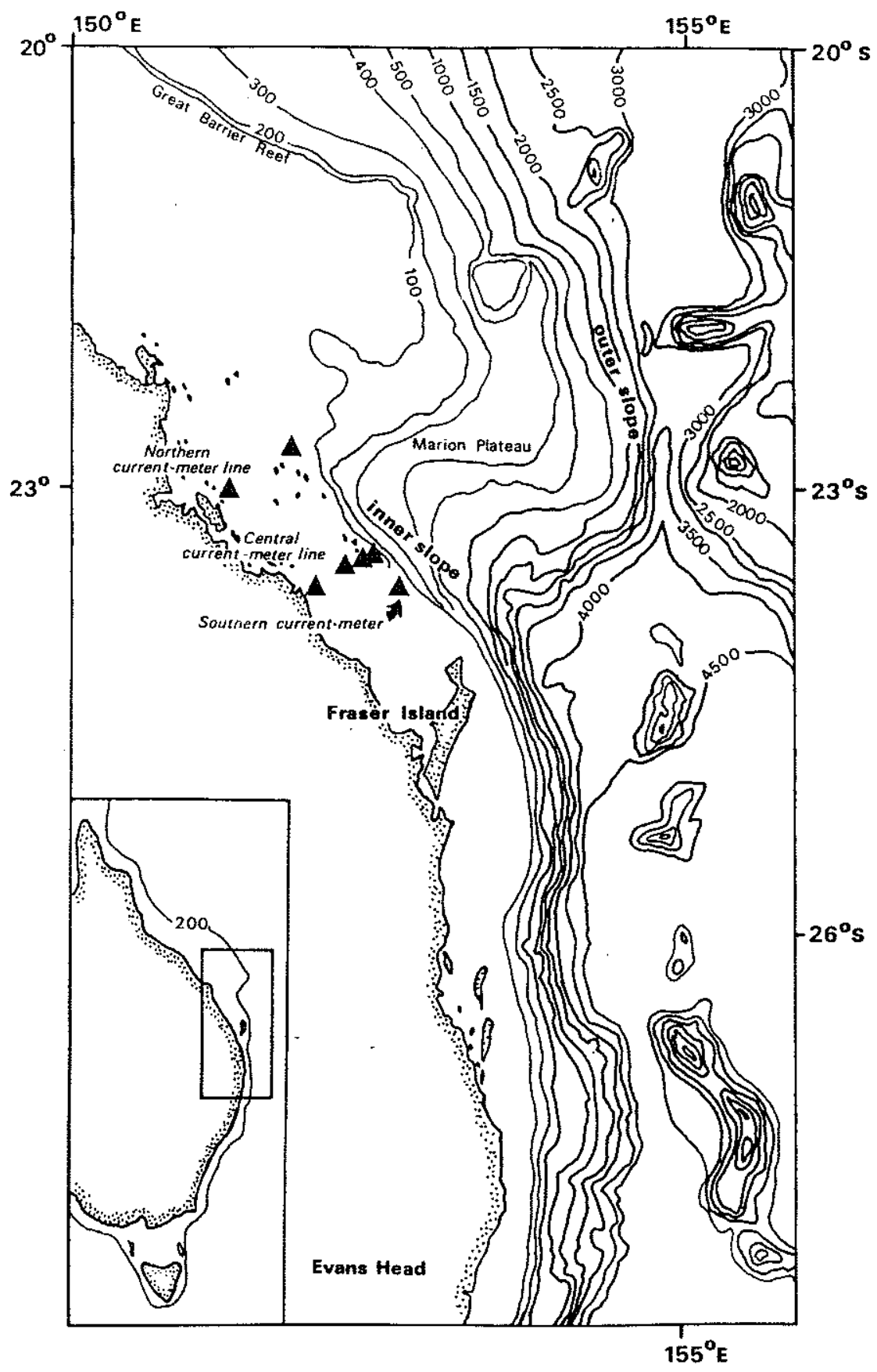

Figure 6-1: The region of the East Coast of Australia where observations by Griffin and Middleton (1986) reveal the presence of scattered CTWs. The sites where current meters were deployed by GM are indicated by the solid triangles. 
a geographic origin, namely FI, under the assumption that FI would act as a barrier to any remotely-generated CTws. Contrary to this hypothesis, GM concluded that local wind-driving could not account for the substantial energy observed at subinertial frequencies and that, instead, the observed low-frequency currents were due principally to waves which propagated into the study region from south of FI. Evidence for the presence of freely-propagating CTWs within the study region comes from a modal decomposition of the across-shelf structures of the alongshelf currents at the central instrument line. Using the fitted mode amplitudes, currents at the southern current-meter site were hindcast by propagating the modes at their predicted phase speeds. Good agreement between the observed and hindcast currents suggests strongly that freely-propagating CTWs account for much of the subinertial frequency variability within the study region. The modal decomposition indicated that the majority of the energy was carried by a second or third mode CTW having a period of approximately 9 days, and a propagation speed of about $0.4 \mathrm{~ms}^{-1}$. Evidence that there is a source for these waves in the form of freely-propagating crws incident upon FI may be found in the results of the Australian Coastal Experiment (ACE) (Freeland et al., 1986), which in part ran concurrently with GM's field study. The ACE results showed the presence of cTWs on the continental shelf between $400 \mathrm{~km}$ and $1000 \mathrm{~km}$ south of FI, and since the shelf topography between FI and Evans Head (the northernmost $\mathrm{ACE}$ instrument line) is only slowly varying, it is probable that free CTws were incident upon FI during GM's instrument deployment. GM establish a connection between these incident waves and the CTWs they observed by demonstrating that there is a statistically significant lagged correlation between the currents at Evans Head and the currents they measured north of FI. The phase difference GM computed was 33 hours, which corresponds to a northward propagation speed of approximately $4.5 \mathrm{~ms}^{-1}$.

Additional support for GM's conjecture that free waves incident from south of FI would be scattered by the irregular topography of the region and thereby generate the waves observed in their data is provided by the results of Chapter 5 . The geography of the FI region is comprised of features which resemble some of the topographic irregularities considered in isolation in Chapter 5. Approaching FI from the south, the shelf width decreases to a narrow neck adjacent to the northern tip of the FI (Figure 6-1). This narrowing is similar in some respects to the topography of Figure 5-4, which scattered up to $13 \%$ of an incident mode 1 CTw into higher 
modes (Table 5-1) and produced an intensification in the alongshelf currents at the narrowest part of the scattering region (Figure 5-21, curve B). To the north of FI, the shelf widens rapidly and the isobaths essentially split to form two 'slopes.' The shelf and 'inner slope,' where GM's instruments were deployed, are separated from the 'outer slope' by the Marion Plateau, which is fairly uniform in depth $(\sim 450 \mathrm{~m}$ ) across its $200 \mathrm{~km}$ width (Figure 6-1). The isobaths of the outer slope move offshore in a manner similar to the least shelf-similar widening topography considered in Chapter 5 (i.e. the reversal of the topography of Figure 5-7), which scattered over $20 \%$ of the incident wave energy (Table 5-6, column D). The topography of the inner slope and shelf is more analogous to that of Figure 5-12 (Table 5-6, column $\mathrm{c}$ ) where the shallow inner shelf widens due principally to a change in the position of the coast. Considered in isolation in Chapter 5, each of these topographic variations caused appreciable scattering of CTW energy, and a significant modulation of the flow field both within, and immediately downstream from, the scattering region. This suggests that it is indeed likely that free waves encountering the irregular geometry of the FI region would produce an observable signal, in the form of freely-propagating scattered CTWs, on the inner slope and shelf north of FI.

To test this hypothesis more rigorously, a numerical wavetank experiment has been conducted which simulates the scattering of a free CTW pulse by a model topography (Figure 6-2) which approximates closely the geography of the Fi region. Comparison with Figure 6-1 shows that the model topography is somewhat smoother than the actual topography of the region, and that an arbitrary abyssal depth of $3000 \mathrm{~m}$ was chosen. It was necessary to model the promontory of $\mathrm{FI}$ as a rather blunt peninsula in order to avoid prohibitively small spacings in the computational coordinate grid. Apart from this approximation, the model geometry follows that of the FI region quite closely. South of FI, and north of the Marion Plateau, the topography has been extended uniformly alongshelf. This makes it possible to limit the scattering observed in the simulation to that generated solely by the geography of the Fi region. Church et al. (1986a) observed $N^{2}$ values of roughly $5 \times 10^{-6} s^{-2}$ below $500 \mathrm{~m}$ depth in the ACE region. This value, assumed constant throughout the water column, was adopted for the Fi simulation. The shallow thermocline ( $\sim 100 \mathrm{~m}$ depth) observed by GM in their CTD casts over the Marion Plateau was not included in the simulation due to difficulties that the PEM has resolving such a near-sufrace feature in the deepest regions of the computational domain. The omission of the 


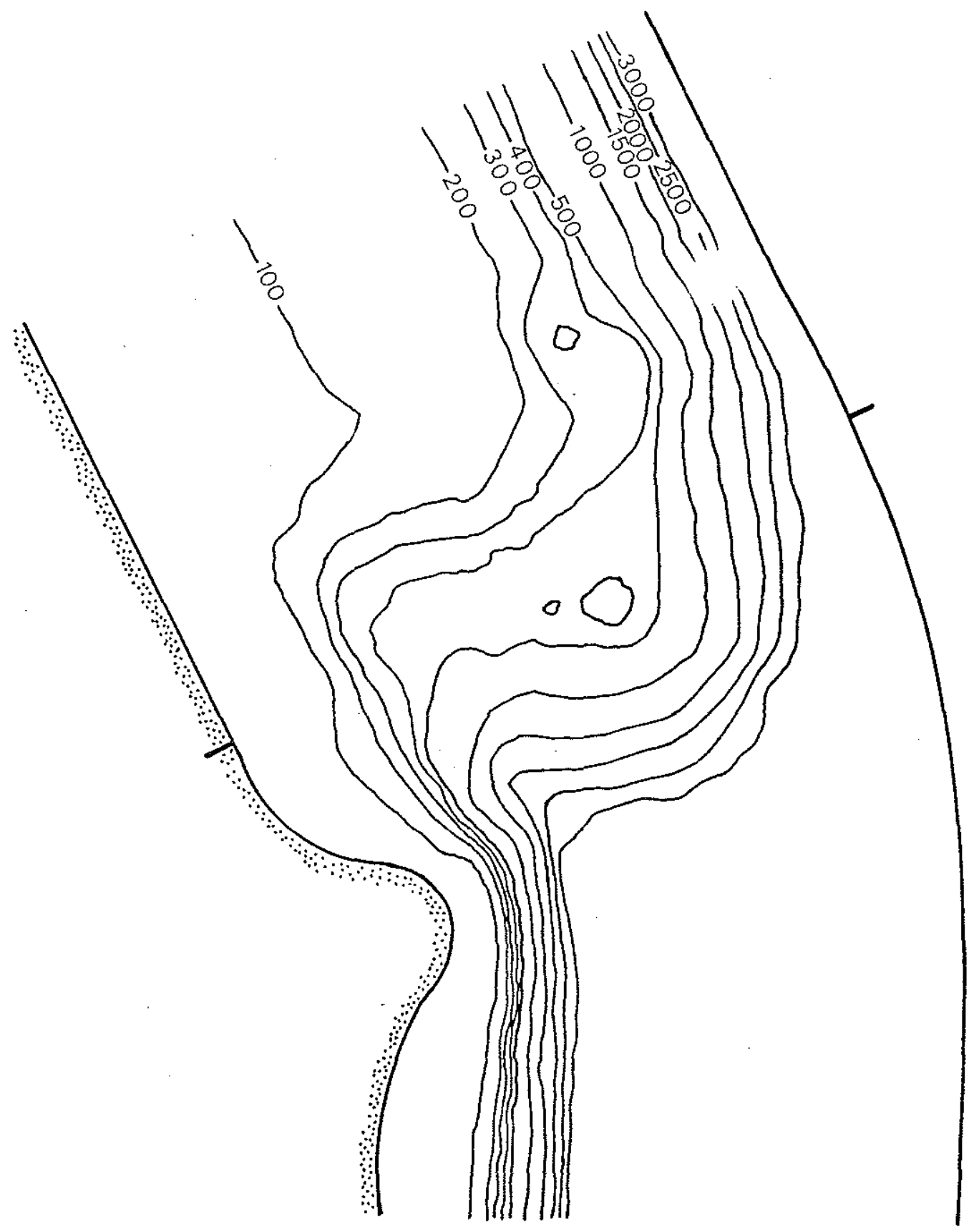

Figure 6-2: The topography used in the simulation of cTw scattering in the FI region. The topography has a smoothed coastline, and assumes a uniform abyssal depth of $3000 \mathrm{~m}$, but otherwise approximates closely the true topography (Figure 6-1). 
thermocline has negligible effect on the free wave dispersion relation (results not shown) but does create some differences in the details of the modal structures. These are discussed later.

The choice of an appropriate input for the simulation is not straightforward. GM noted a peak in the subinertial frequency spectra of their observations at around 9 days. However, during those periods of the instrument deployment which were dominated by energy in the 6-10 day frequency band, the observed currents had a nonzero northward mean, and therefore could not be simply the signature of a continuous train of cTws. This could be due to the presence of a northward mean flow unrelated to the CTWs, or to the observed waves being essentially nondispersive CTW pulses with predominantly northward alongshelf current signatures. For the purposes of this discussion it was assumed that the latter was the case, and a solitary mode $1 \mathrm{CTW}$ pulse representing the crest of a nine-day-period CTW was chosen as the input to the simulation. A mode 1 input wave was assumed because it is the phase speed of mode 1 $\left(3.44 \mathrm{~ms}^{-1}\right)$ which most closely corresponds to the observed speed $\left(4.5 \mathrm{~ms}^{-1}\right)$ at which current fluctuations propagated into the study region from Evans Head. At this frequency $(0.13 f)$ the CTWs are only weakly dispersive (Figure 6-3), so, as shown in Appendix A, the amplitudes of the modes scattered by a pulse should not differ significantly from those generated by a continuous wavetrain. The results should therefore be similar qualitatively to those which would be obtained for many other input waveforms.

The output of the simulation was analysed in the same manner as the results of the previous numerical experiments, i.e. by performing a fit of the calculated alongshelf currents to a set of freely-propagating scattered CTW modes. The alongshelf location at which the mode fit was performed (indicated by the single tick marks in Figure 6-2) was chosen to correspond to the location of GM's central instrument line. Performing the mode fit at such a location, i.e. where the topography is changing alongshelf, is not strictly valid because the assumption that the local CTW modes have a simple-harmonic alongshelf dependence (section 4.4) cannot be made. Nevertheless, the analysis was performed in this manner to facilitate as direct a comparison as possible to the modes GM fitted to their observations. Computation of the free-wave modal structures and phase speeds at this location reveals some interesting properties of the 'dual slope' topography north of FI. The across-shelf structures of the first six modes are slown in Figure 6-4. The structure of mode 1 takes a maximum at the coast, decays slightly over the 


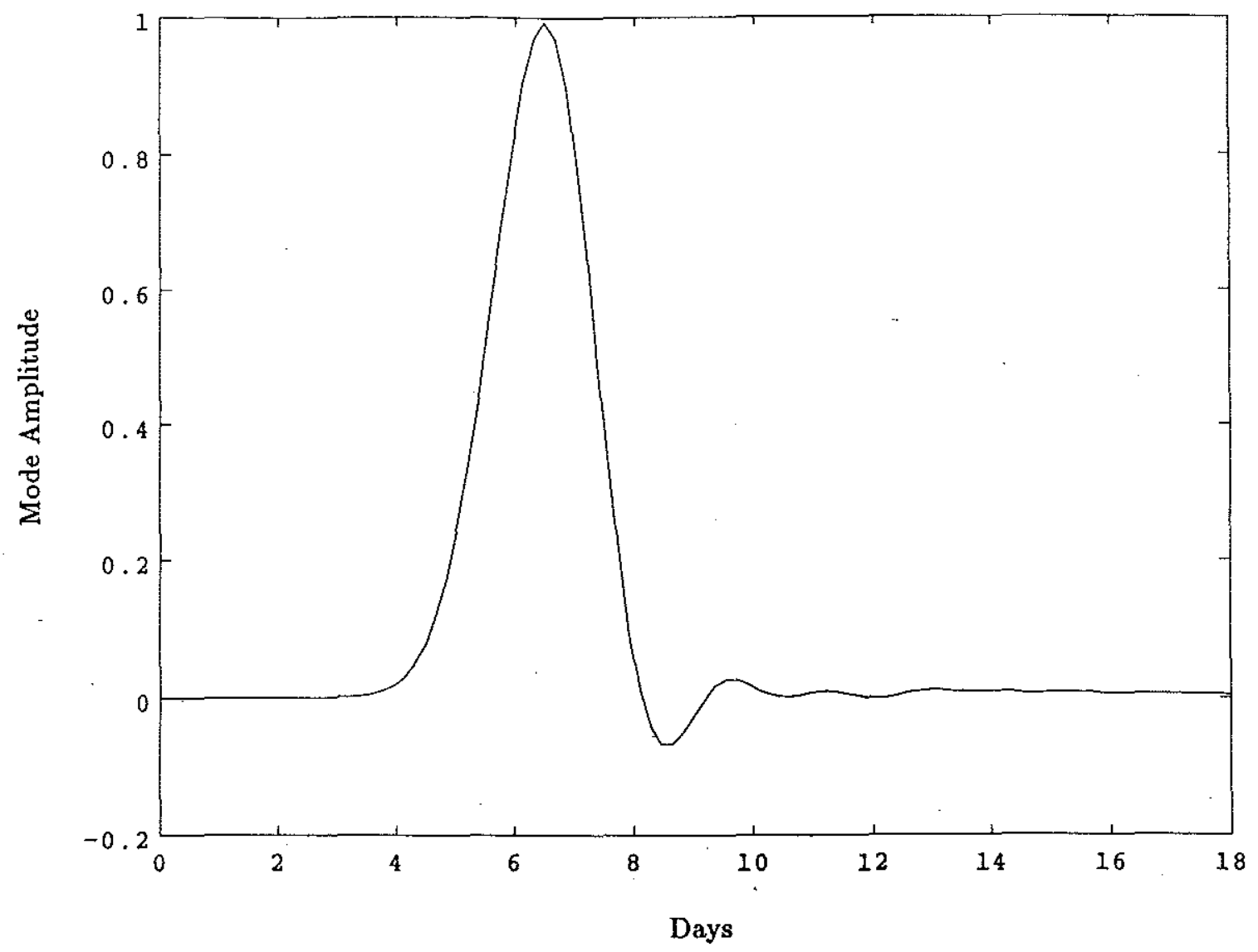

Figure 6-3: The propagation of a simulated CTw pulse along a uniform shelf with the same depth profile as the shelf south of FI. The figure shows the alongshelf velocity at the coast $1200 \mathrm{~km}$ downstream from the numerical wavemaker. There has been no loss in the peak amplitude and only a weak dispersive tail trails the pulse.

inner slope, remains relatively constant over the Marion Plateau, and does not take its zero crossing until over the outer slope. The structures of the higher modes fall into two distinct classes: modes 3,4 and 5 are concentrated over the outer slope, while modes 2 and 6 are trapped closely against the inner shelf and slope. None of these modes has any appreciable signal over the Marion Plateau. GM calculated their modal structures under the assumption that the wave motions they observed were confined to the inner shelf and slope, and were unaffected by the topography of the outer slope due to the broadness of the Marion Plateau. Their analysis therefore missed those modes in Figure 6-4 whose structures are concentrated over the outer slope. Nevertheless, there appears to be a direct correspondence between GM's 


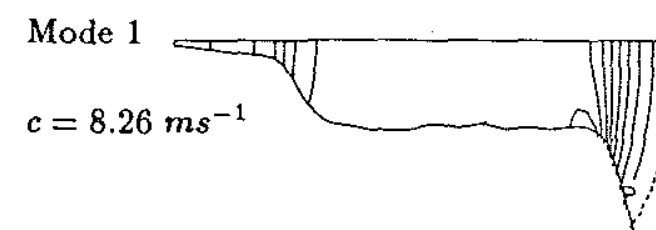

\section{Mode 2

$$
c=2.94 \mathrm{~ms}^{-1}
$$

Mode 3

$$
c=1.48 \mathrm{~ms}^{-1}
$$

$$
\begin{aligned}
& \text { Mode } 4 \\
& c=0.77 \mathrm{~ms}^{-1}
\end{aligned}
$$

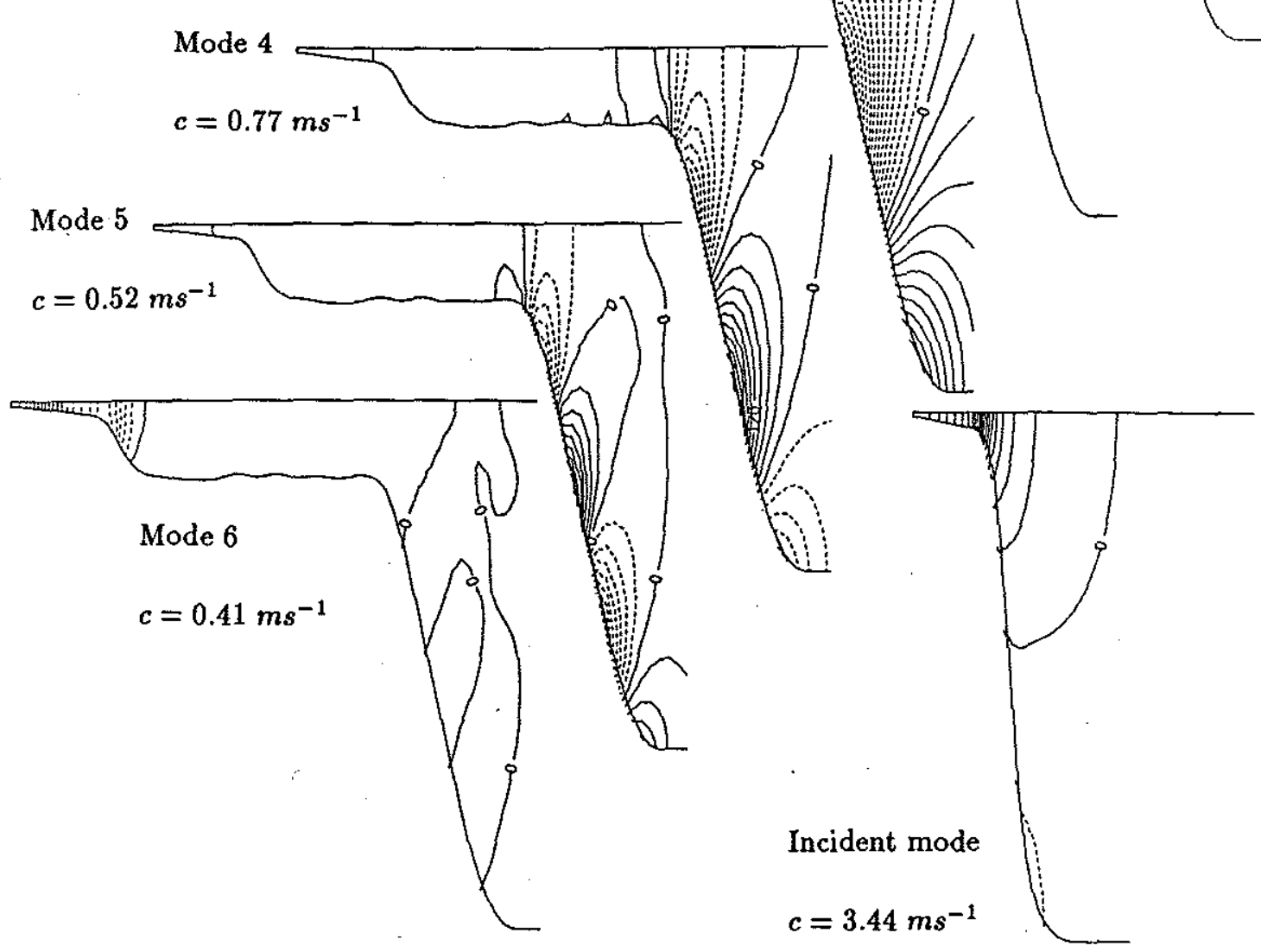

Figure 6-4: Across-shelf modal structures of the incident wave (mode 1) and the first six transmitted modes at the alongshelf location indicated by the tick marks in Figure 6-2. 
modes (Figure 6-5) and the inner slope modes of Figure 6-4. In particular, GM's mode 1, which has a phase speed of $3.1 \mathrm{~ms}^{-1}$ and a zero crossing at mid-depth on the inner slope, appears to correspond to mode 2 in Figure 6-4, which has a phase speed of $2.94 \mathrm{~ms}^{-1}$. Mode 6 of Figure 6-4 has a phase speed of $0.41 \mathrm{~ms}^{-1}$, which is virtually identical to GM's mode $3\left(0.4 \mathrm{~ms}^{-1}\right)$, while the across-shelf structure of mode 6 resembles that of GM's mode 2, with zero crossings on the shelf and at the foot of the inner slope. This ambiguity is not of particular concern given that GM were unable to distinguish between the contribution of their modes 2 and 3 to the observed currents. Furthermore, the structure of GM's mode 2 was particularly sensitive to the assumed depth of the thermocline (D. Griffin, pers. comm.) which has been wholly omitted from the simulation.

The time series of the amplitudes of the scattered modes returned by the least-squares mode fit are shown in Figure 6-6. It can be seen that the incident wave pulse scatters primarily into two pulses (modes 1 and 2) of similar shape to the incident pulse, in the manner predicted in Chapter 3 (and Appendix A). The arrival of the first two pulses is followed by a tail of predominantly higher mode waves. These may be the signature of waves generated at the beginning of the region of irregular topography which are scattered repeatedly throughout the finite length of the scattering region, or alternatively, this tail could simply result from the more dispersive nature of the higher mode pulses.

The magnitude of the mode 1 pulse shows that the majority of the incident mode energy remains in mode 1 , as was the case in the results of Chapter 5 . This raises a question as to why GM did not observe this mode in their data. In Figure 6-4 it can be seen that the structures of modes 1 and 2 are quite similar over the inner slope and shelf. Since GM did not have any instruments deployed over the outer slope, where the structures of these modes differ, it is unlikely that their analysis would be able to discriminate between the signals of the two modes. Furthermore, a mode 1 and mode 2 wave generated simultaneously at FI would reach GM's northernmost current-meter less than half a day apart - a time separation likely too small to be resolved by GM's spectral analysis. The strong peak of the mode 2 pulse shows that a significant amount of the scattered energy propagates along the inner slope into GM's study area. The energy in the remaining higher modes is small. In particular, mode 6 , which corresponds to GM's mode 2 or 3 , carries little of the scattered energy flux. Nevertheless, this 


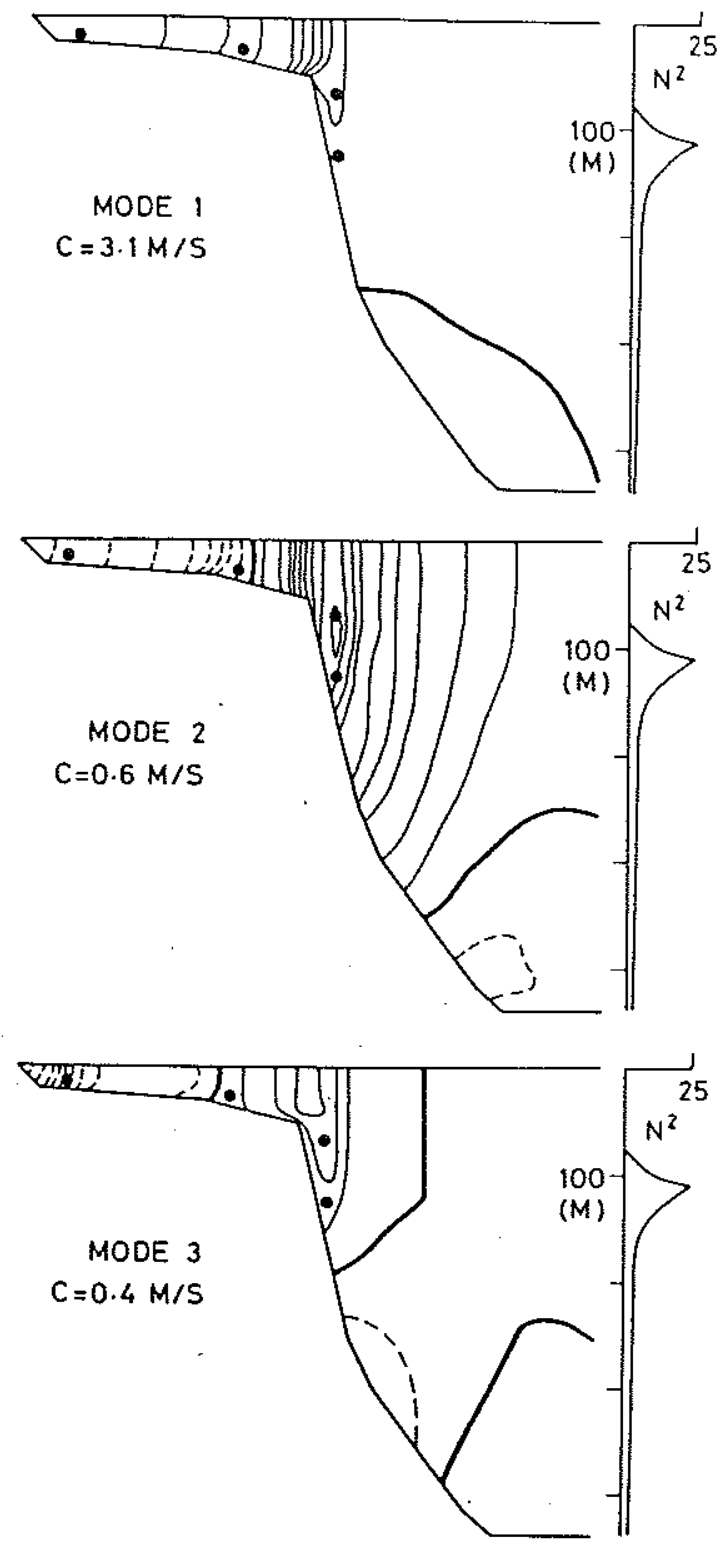

Figure 6-5: Modal structures of the alongshelf velocity of the first three Crw modes computed by Griffin and Middleton (1986) at the location corresponding to their central current-meter line (Figure 6$1)$. 


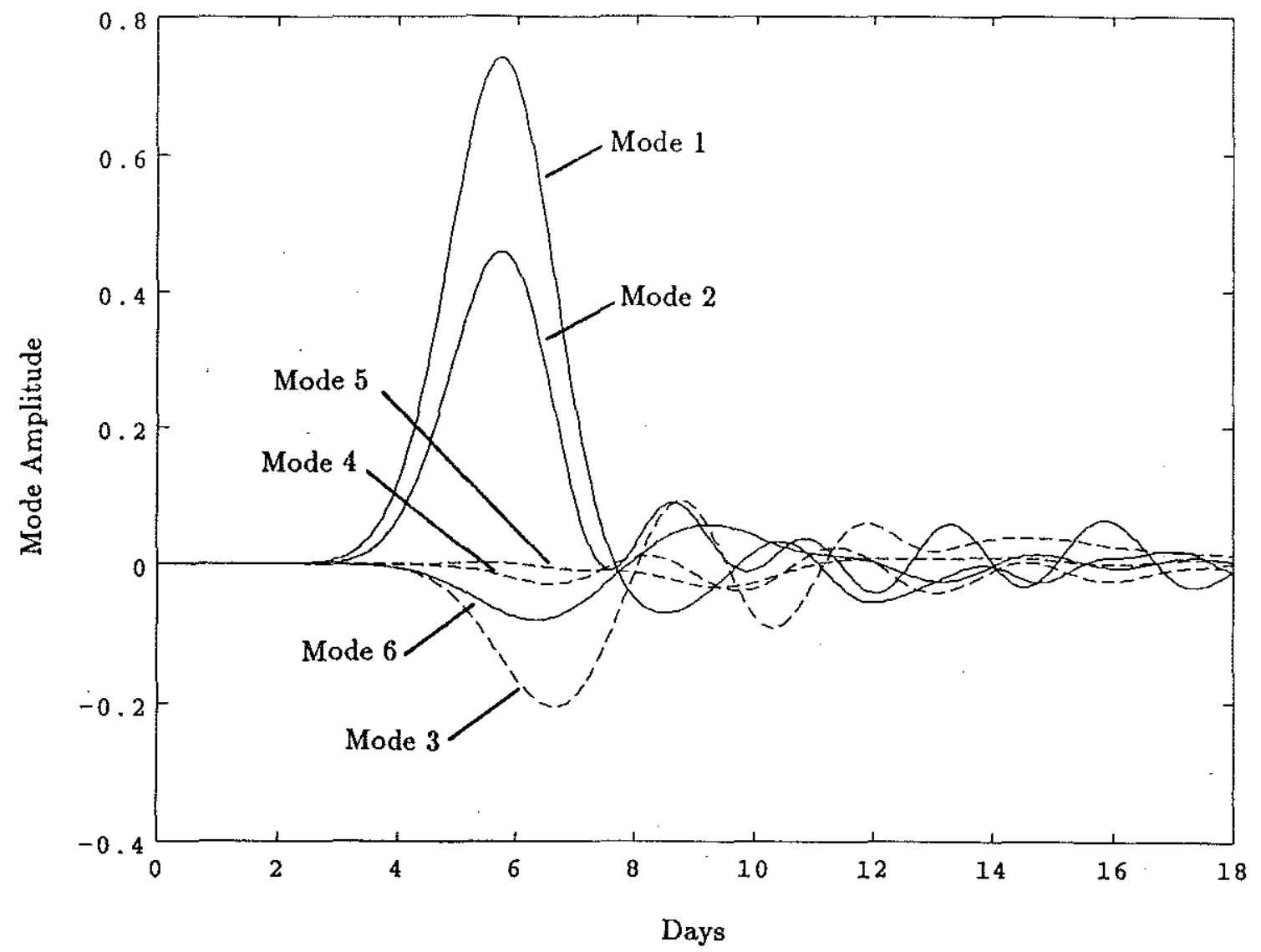

Figure 6-6: Time series of the amplitudes of the transmitted modes calculated by the least-squares mode fit for the FI case study simulation.

mode has a strong signal in the currents on the inner slope and shelf. Figure 6-7 shows the contribution of each mode to the total surface alongshelf velocity at the coast, computed by multiplying the mode amplitude time series (Figure 6-6) by the values of the mode structures at the coast. The velocity at the coast due to the energetic mode 1 wave is quite weak, providing a further reason why its signal should not be particularly evident in GM's observations. By far the strongest signals are those due to the inner slope modes; modes 2 and 6 . The phase lag between their arrivals at the mode fit section ( $\sim 0.5$ days) is consistent with the difference in their phase speeds if, as would seem reasonable, the waves were generated at the tip of FI. The coastal currents induced by these two modes largely cancel, in agreement with GM's observations that their modes 1 and 2 added in near anti-phase near the coast. Conversely, at the inner shelf 


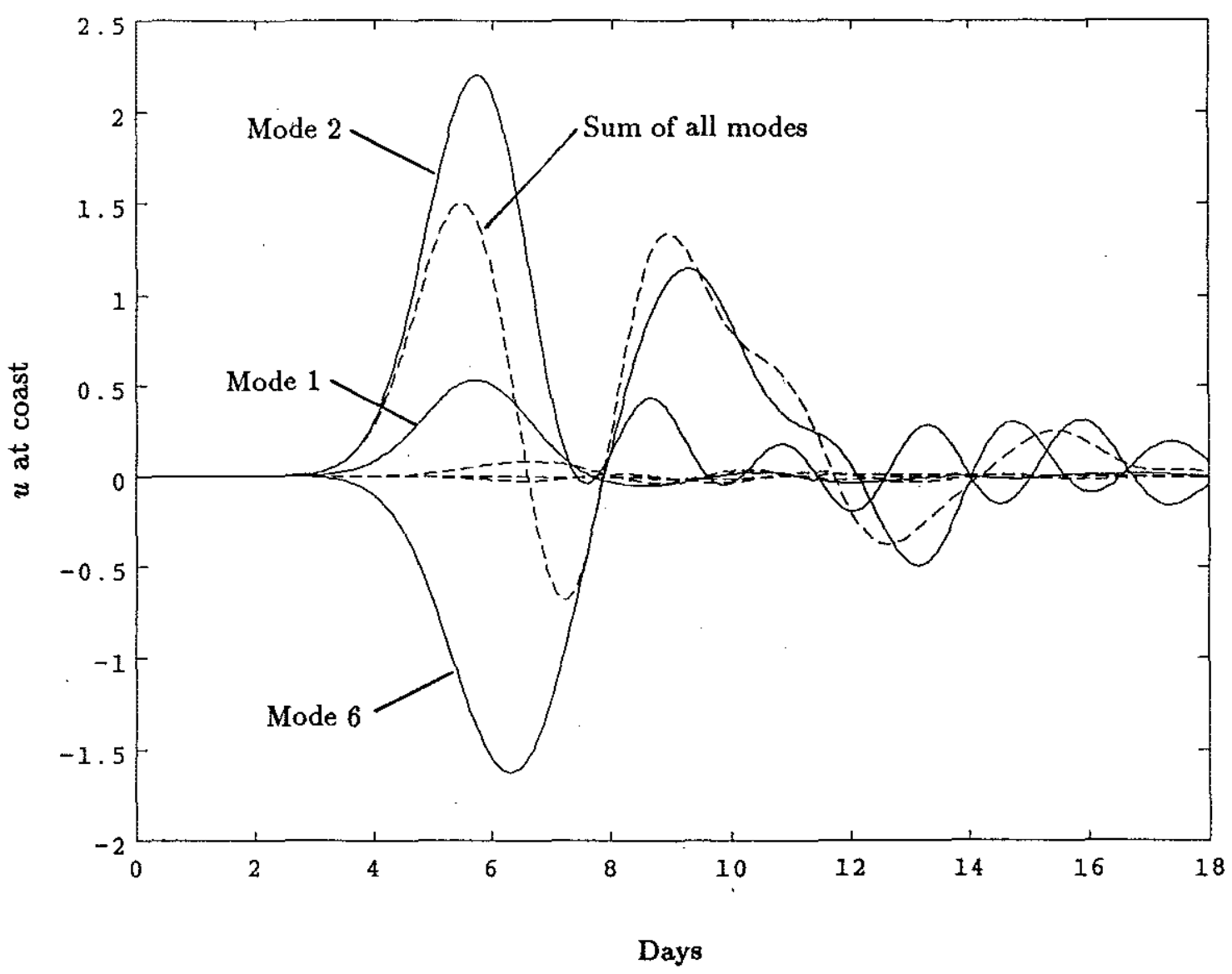

Figure 6-7: Surface alongshelf current at the coast at the location corresponding to GM's central instrument line. The current due to each mode is computed by taking the product of the mode amplitude time series (Figure 6-6) and the value of the individual modal structures (Figure 6-4) at the coast. Dashed line shows the superposition of individual contributions.

break the currents induced by the modes reinforce (Figure 6-8). This is also in agreement with GM's observations; namely, that the subinertial frequency currents at their central instrument line were most energetic at the shelf-break. This qualitative similarity between the features of the simulation and GM's observations lends strong support to GM's hypothesis that the waves they observed were scattered from freely-propagating CTWs originating south of FI.

Some other interesting features of the simulation may be seen in Figures 6-9 and 6-10, which show time histories of the surface alongshelf velocity, and surface velocity vectors, respectively, as the pulse propagates through the scattering region. The figures have been drawn in a 'northern hemisphere' geometry so that they can be compared with similar plots in Chapter 5. 




Figure 6-8: Surface alongshelf current at the inner shelf break at the location corresponding to GM's central instrument line. The currents are computed in the same manner as Figure 6-7.

As the pulse reaches the promontory of $\mathrm{FI}$ intense alongshelf currents are generated. These achieve a maximum value three times greater than the coastal currents associated with the incident wave (Figure 6-11). The separation of the individual pulses as they reach the uniform topography north of the Marion Plateau is also apparent.

In summary, the results of this simulation show that realistic shelf geometries can scatter significant levels of CTW energy, and that the scattered waves can have an appreciable signal in current-meter observations made on the continental shelf. $\mathrm{GM}$ 's observation of what is actually a very high mode (mode 6) free wave with a low phase speed $\left(0.4 \mathrm{~ms}^{-1}\right)$ reinforces the point noted in Chapter 3 that along irregular coastlines it is necessary to account for the possibility that crw scattering processes may be in effect, if oceanographic observations are 


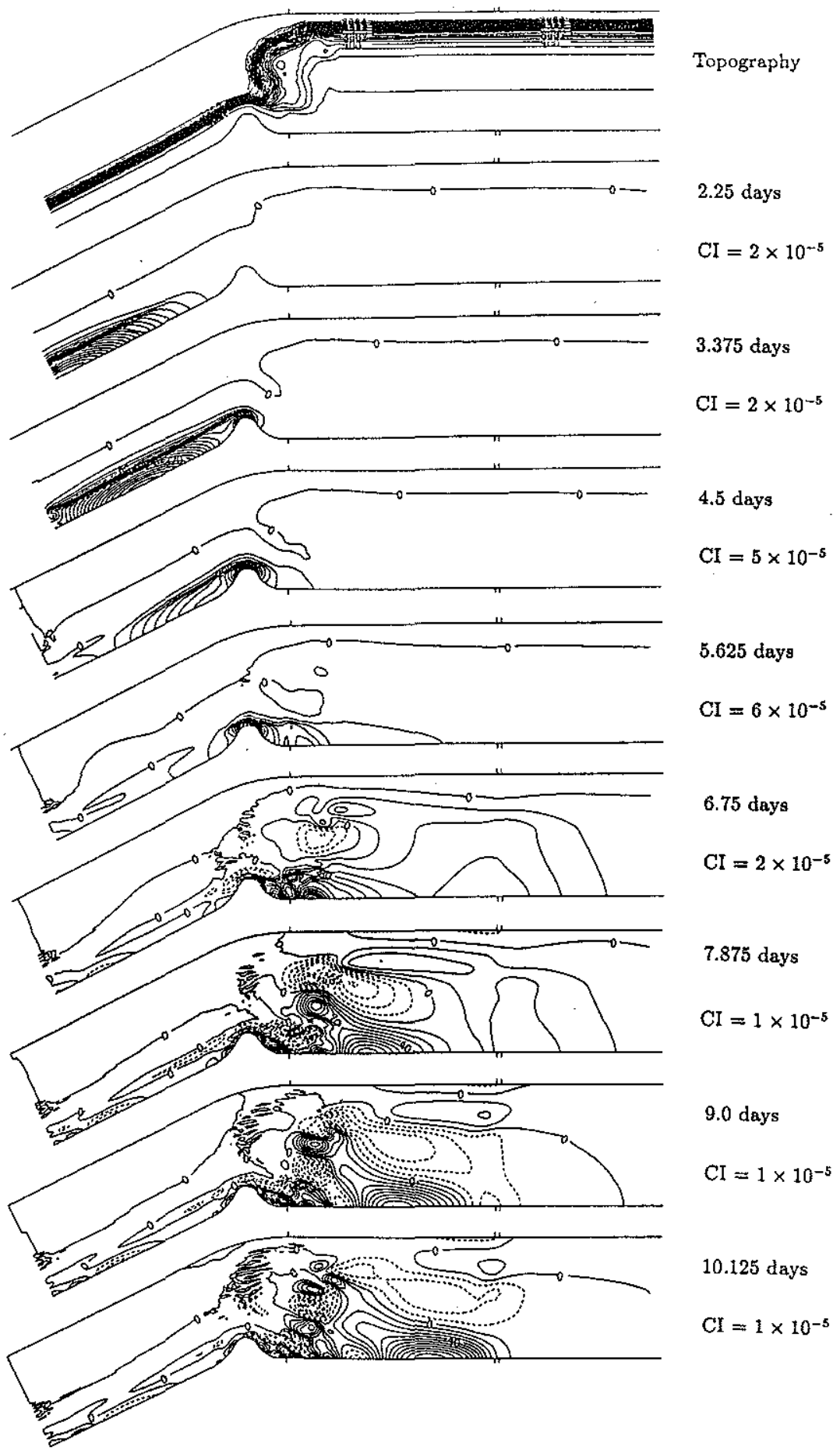

Figure 6-9: Surface alongshelf velocity at different times during the FI scattering simulation. CI denotes contour interval. Note that the plots are presented in a reflected 'northern hemisphere' geometry for comparison with similar figures in Chapter 5. 


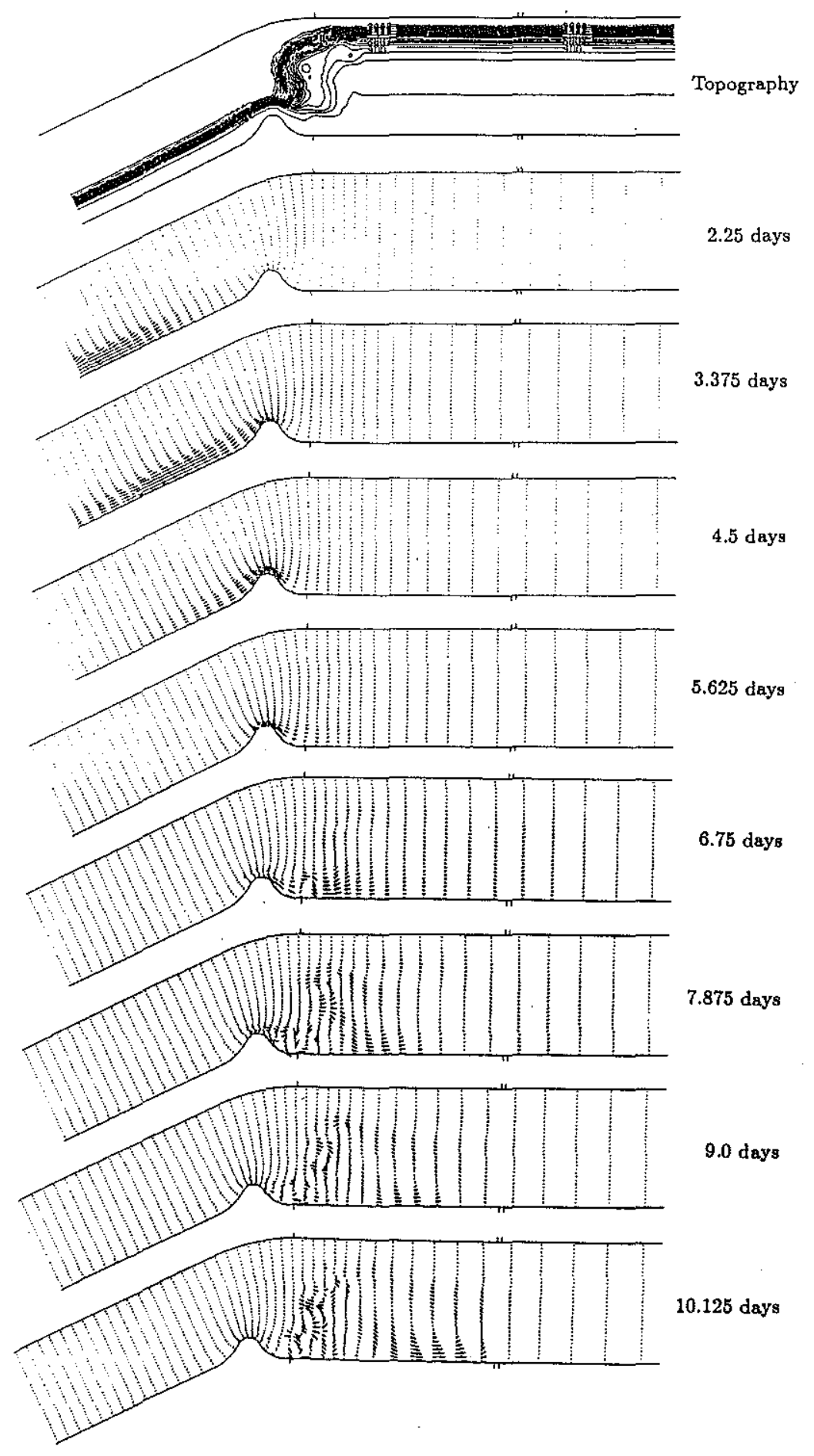

Figure 6-10: Surface velocity vectors at different times during the Fi scattering simulation. Note that the plots are presented in a reflected 'northern hemisphere' geometry for comparison with similar figures in Chapter 5. 


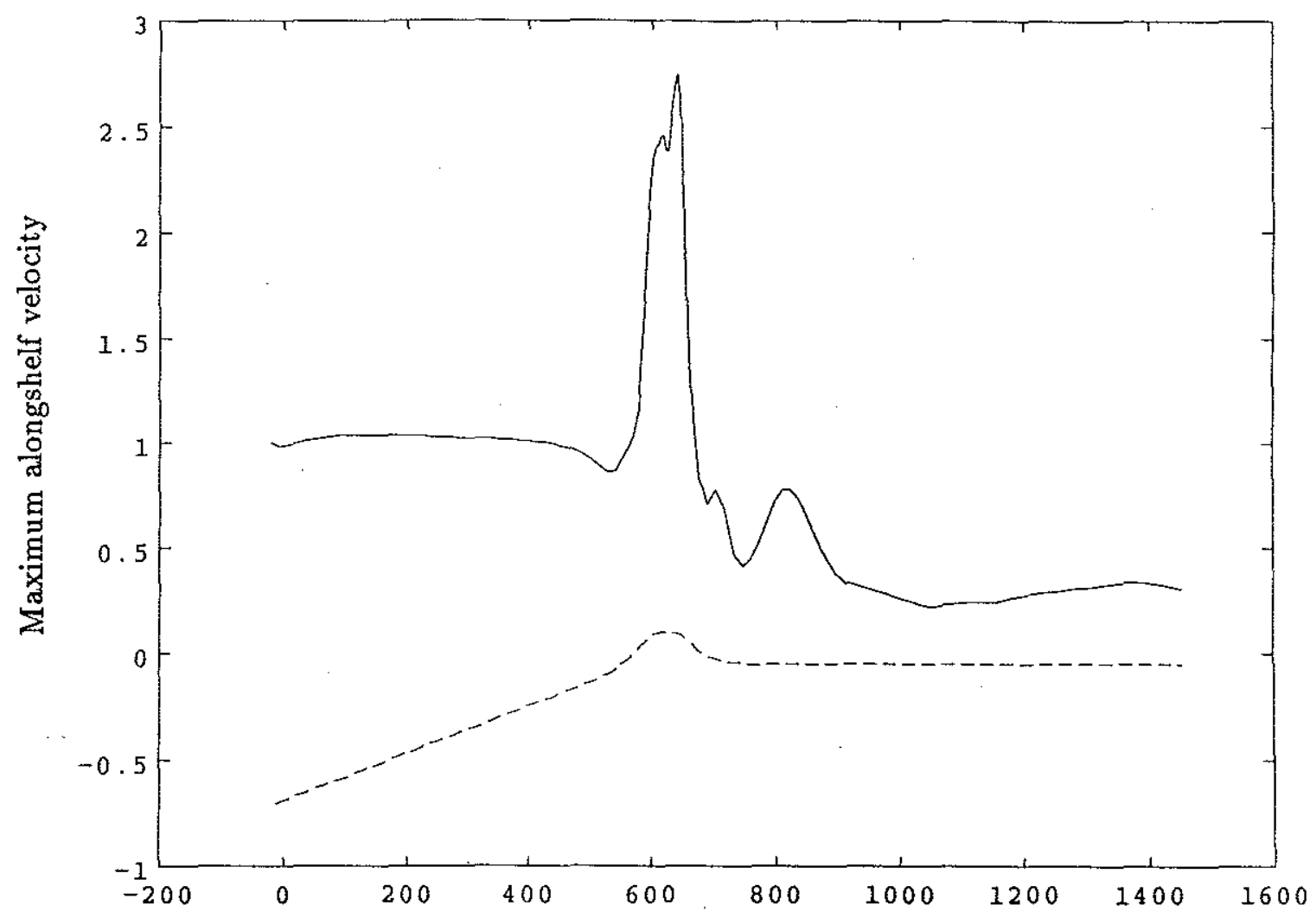

Alongshelf distance $(\mathrm{km})$

Figure 6-11: Maximum surface alongshelf velocity at the coast for the F1 scattering simulation.

to be interpreted correctly. The success of the model simulation in reproducing features of GM's observations suggests that simulations of free CTW scattering may be useful for predicting features of scattering processes on other continental shelves of interest, and may prove to be a useful tool for improving the design of field experiments, and the interpretation of their results. 


\section{Chapter 7}

\section{Summary}

Coastal-trapped waves propagate along many of the continental shelves of the world's oceans and can transmit fluctuations in sea-level and current over vast distances. Along relatively straight coasts, where the longwave approximation can be made, wind-driven CTw motions have been hindcast with some success by application of the first-order wave equation method. This method incorporates variations in botton topography and coastline only through slow alongshelf changes in the wave parameters. However, most coastlines vary significantly over spatial scales much shorter than CTW length scales, thereby introducing the possibility that appreciable scattering of CTW energy from one mode into others can occur over quite short alongshelf distances.

This study has examined how freely-propagating CTWs are scattered by large variations in coastline and topography occuring over alongshelf distances comparable to the shelf width in a realistically stratified coastal ocean. Particular attention has been paid to the role of stratification because, as shown, the introduction of even modest stratification can eliminate reflected free-waves with large wavenumbers which occur, theoretically, in a barotropic ocean. For this reason, discussion of the analytical solution presented in Chapter 3 for the scattering of a barotropic shelf wave by a discontinuity in shelf width, is restricted to the range of model parameters over which energy transmission is nearly $100 \%$. The solution shows there is a substantial transfer of energy to modes other than that of the incident wave. The transmitted mode most readily excited is that which has the across-shelf structure most closely coinciding with that of the incident wave. For a widening shelf, energy is therefore readily transferred to 
higher modes. It was shown that the scattering is only weakly dependent on the wave frequency when the frequency is low, and that as a result, a non-dispersive shelf wave 'pulse' of limited alongshelf extent scatters into a train of similarly shaped waves of all allowable modes, each propagating at its own free-wave speed. Of relevance to observational studies is the result that the interaction of multiple scattered modes with differing wavelengths, phase speeds, and acrossshelf structures, modulates the magnitude and phase progression of the flow field downstream of the scattering region. Further evidence for the confounding role that scattering may play in the interpretation of shelf wave observations is the result that the waves scattered from multiple incident waves of different modes can interfere to enhance the energy in some modes while reducing that in others. This has the effect of making the apparent scattering strength of a topography dependent not only on the geometry, but also on the incident wave pattern.

The principal restriction incurred in the analytical study, through the necessity to avoid solutions showing substantial reflection, is the exclusion of any discussion of narrowing shelves. To overcome this limitation, and to enable the consideration of more realistic topographic variations, a numerical model which accomodates arbitrary density stratification, bathymetry, and coastline, was employed. Numerical simulations were conducted of the scattering of crws by a set of topographic and coastline variations which are representative of many continental shelves. The strength of the scattering observed, as measured by the proportion of the incident energy flux scattered into modes other than that of the incident wave, was found to be proportional to a topographic warp factor which estimates the extent to which the topography departs from shelf-similarity. The scattering induced by non-shelf-similar topographic variations is amplified by density stratification. A comparison of the effects of widening and narrowing topographies showed that the amplitudes of the freely-propagating transmitted modes generated by "reciprocal" narrowing and widening topographies are quite similar. Within the scattering region itself, the strengths of the scattered-wave-induced currents exhibit substantial variation over short spatial scales. On both widening and narrowing shelves, there is generally a marked intensification of the flow within the scattering region, and significant differences in the directions of the currents at points separated by a few tens of kilometers indicate the occurence of rapid variations in phase. On narrowing shelves, the influence of the scattering can extend upstream into the region of uniform topography. 
The understanding of CTW scattering processes gained from the study was applied to the interpretation of a simulation of CTW scattering at a site on the East Coast of Australia where observations made by Griffin and Middleton (1986) suggest the presence of scattered freelypropagating CTWs. The simulation supports Grifin and Middleton's (1986) conjecture that the waves they observed were the signature of remotely-forced free Crws scattered by the abrupt topographic variations of their study region. This confirms that realistic shelf geometries can scatter significant levels of CTW energy, and that the scattered waves can have an appreciable signal in current-meter observations made on the continental shelf. Griffin and Middleton's (1986) observation of what is actually a very high mode (mode 6) free wave with a low phase speed $\left(0.4 \mathrm{~ms}^{-1}\right)$ reinforces the point noted above, that along irregular coastlines it is necessary to account for the possibility that CTw scattering processes may be in effect if oceanographic observations are to be interpreted correctly. The success of the model simulation in reproducing features of Griffin and Middleton's (1986) observations suggests that simulations of free CTW scattering may be useful for predicting features of scattering processes on other continental shelves of interest, and may prove to be a useful tool for improving the design of field experiments, and the interpretation of their results. 


\section{Appendix A}

\section{Scattering of a Barotropic Shelf}

\section{Wave Pulse}

Consider an incident barotropic shelf wave of limited alongshelf extent represented by a superposition of periodic waves with a continuum of wavelengths:

$$
\psi_{I}(x, y, t)=\phi_{1 I}(x) \int_{-\infty}^{\infty} A(\hat{\ell}) e^{i \hat{\ell}(y-\hat{c} t)} d \hat{\ell}
$$

Provided the amplitude spectrum $A(\hat{\ell})$ is limited to small wavenumbers, the phase speed $\hat{c}=\omega / \hat{\ell}$ will be a constant dependent only on the shelf geometry and the 'pulse' will maintain a permanent form as it propagates. At the low frequency, small wavenumber limit, it has been demonstrated in section 3.2 that a single incident continuous wave scatters into a train of transmitted waves of amplitudes $B_{q}$ with the $B_{q}$ being functions only of the shelf geometry. For a unit amplitude incident wave, this scattering process may be summarized as:

$$
\phi_{1 I}(x) e^{i\left(\hat{\ell}_{y}-\omega t\right)} \rightarrow \sum_{q=1}^{\infty} B_{q}\left(\lambda, L_{1}, L_{2}\right) \phi_{2 q}(x) e^{i\left(\ell_{2 q} y-\omega t\right)}
$$

Since the scattering model is linear, it follows that the pulse will scatter as

$$
\psi_{I}(x, y, t) \rightarrow \int_{-\infty}^{\infty} \sum_{q=1}^{\infty} A(\hat{\ell}) B_{q}\left(\lambda, L_{1}, L_{2}\right) \phi_{2 q}(x) e^{i\left(\ell_{2 q} y-\omega t\right)} d \hat{\ell}
$$

Noting that $\omega=\hat{c} \hat{\ell}=c_{2 q} \ell_{2 q}$, this simplifies to

$$
\psi_{I}(x, y, t) \rightarrow \sum_{q=1}^{\infty} B_{q} \phi_{2 q} \int_{-\infty}^{\infty} A(\hat{\ell}) e^{i\left(\hat{c} / c_{2 q}\right) \ddot{\ell}\left(y-c_{2 q} t\right)} d \hat{\ell}
$$


Comparison with (A.1) shows that the wave components combine to produce a train of pulses of differing modes, each with shape similar to that of the incident wave but stretched in the alongshelf direction by a factor $c_{2 q} / \hat{c}$. Each pulse travels at its own non-dispersive free wave speed $c_{2 q}$. 


\section{References}

Allen, J.S., 1976: Continental shelf waves and alongshore variations in bottom topography and coastline. Journal of Physical Oceanography, 6, 864-878.

Allen, J.S., 1980: Models of wind-driven currents on the continental shelf. Annual Review of Fluid Mechanics, 12, 389-433.

Battisti, D.S. and B.M. Hickey, 1984: Application of remote wind-forced coastal trapped wave theory to the Oregon and Washington coasts. Journal of Physical Oceanography, 14, 887-903.

Brink, K.H., 1980: Propagation of barotropic continental shelf waves over irregular bottom topography. Journal of Physical Oceanography, 10, 765-778.

Brink, K.H., 1982a: The effect of bottom friction on low-frequency coastal trapped waves. Journal of Physical Oceanography, 12, 127-133.

Brink, K.H., 1982b: A comparison of long coastal trapped wave theory with observations off Peru. Journal of Physical Oceanography, 12, 897-913.

Brink, K.H., 1986: Scattering of long coastal-trapped waves due to bottom irregularities. Dynamics of Atmospheres and Oceans, 10, 149-164.

Brink, K.H. and J.S. Allen, 1978: On the effect of bottom friction on barotropic motion over the continental shelf. Journal of Physical Oceanography, 8, 919-922.

Brink, K.H. and J.S. Allen, 1983: Reply (to T.J. Simons' comments on K.H. Brink and J.S. Allen, J. Phys. Oceanogr., 8, 919-922, 1978). Journal of Physical Oceanography, 13, 149-150.

Brink, K.H., D.C. Chapman and G.R. Halliwell, 1987: A stochastic model for wind-driven currents over the continental shelf. Journal of Geophysical Research, 92, 1783-1797.

Buchwald, V.T., 1968: The diffraction of Kelvin waves at a corner. Journal of Fluid Mechanics, 31, 193-205. 
Buchwald, V.T., 1977: Diffraction of shelf waves by an irregular coastline. Waves on Water of Variable Depth, D.G. Provis and R. Radok, Eds., Lecture Notes in Physics, Vol. 64, Springer and Australian Academy of Sciences, 235 pp.

Buchwald, V.T. and J.K. Adams, 1968: The propagation of continental shelf waves. Proceedings of the Royal Society, A, 305, 235-250.

Chao, S.-Y., L.J. Pietrafesa and G.S. Janowitz, 1979: The scattering of continental shelf waves by an isolated topographic irregularity. Journal of Physical Oceanography, 9, 687-695.

Chapman, D.C., 1983: On the influence of stratification and continental shelf and slope topography on the dispersion of subinertial coastally trapped waves. Journal of Physical Oceanography, 13, 1641-1652.

Chapman, D.C., 1987: Application of wind-forced, long, coastal-trapped wave theory along the California coast. Journal of Geophysical Research, 92, 1798-1816.

Church, J.A., H.J. Freeland and R.L. Smith, 1986a: Coastal-trapped waves on the East Australian continental shelf, Part I: Propagation of modes. Journal of Physical Oceanography, 16, 1929-1943.

Church, J.A., N.J. White, A.J. Clarke, H.J. Freeland and R.L. Smith, 1986b: Coastal-trapped waves on the East Australian continental shelf, Part II: Model verification. Journal of Physical Oceanography, 16, 1945-1957.

Clarke, A.J., 1977a: Observational and numerical evidence for wind-forced coastal trapped long waves. Journal of Physical Oceanography, 7, 231-247.

Clarke, A.J., 1977b: Wind-forced linear and non-linear Kelvin waves along an irregular coastline. Journal of Fluid Mechanics, 83, 337-348.

Clarke, A.J. and S. Van Gorder, 1986: A method for estimating wind driven frictional, timedependent, stratified shelf and slope water flow. Journal of Physical Oceanography, 16, 1013-1028.

Cox, M.D., 1985: An eddy-resolving numerical model of the ventilated thermocline. Journal of Physical Oceanography, 15, 1312-1324. 
Davis, A.M.J., 1981: The scattering by a headland of the dominant continental shelf wave. Philosophical Transactions of the Royal Society of London A, 303, 383-431.

Davis, A.M.J., 1983: Shelf similar topographies for free continental shelf waves. Geophysical and Astrophysical Fluid Dynamics, 23, 321-331.

Dorr, A. and R. Grimshaw, 1986: Barotropic continental shelf waves on a $\beta$-plane. Journal of Physical Oceanography, 16, 1345-1358.

Enfield, D.B. and J.S. Allen, 1983: The generation and propagation of sea level variability along the Pacific coast of Mexico. Journal of Physical Oceanography, 13, 1012-1033.

Freeland, H.J., F.M. Boland, J.A. Church, A.J. Clarke, A.M.G. Forbes, A. Huyer, R.L. Smith, R.O.R.Y. Thompson and N.J. White, 1986: The Australian Coastal Experiment: A search for coastal-trapped waves. Journal of Physical Oceanography, 16, 1230-1249.

Gill, A.E., 1982: Atmosphere-Ocean Dynamics, 662pp, Acadenic Press, New York.

Gill, A.E. and A.J. Clarke, 1974: Wind-induced upwelling, coastal currents and sea level changes. Deep Sea Research, 21, 325-345.

Gill, A.E. and E.H. Schumann, 1974: The generation of long shelf waves by the wind. Journal of Physical Oceanography, 4, 83-90.

Griffin, D.A. and J.H. Middleton, 1986: Coastal trapped waves behind a large continental shelf island, southern Great Barrier Reef. Journal of Physical Oceanography, 16, 1651-1664.

Grimshaw, R., 1977: The effects of a variable Coriolis parameter, coastline curvature and variable bottom topography on continental shelf waves. Journal of Physical Oceanography, $7,547-554$.

Haidvogel, D.B., J.L. Wilkin and R.E. Young, 1988: A semi-spectral primitive equation ocean circulation model using vertical sigma and horizontal orthogonal curvilinear coordinates. In preparation. To be submitted to the Journal of Computational Physics. 
Halliwell, G.R. and J.S. Allen, 1984: Large scale sea level response to atmospheric forcing along the west coast of North America, Summer 1973. Journal of Physical Oceanography, 14, 864-886.

Halliwell, G.R. and J.S. Allen, 1987: Wavenumber-frequency domain properties of coastal sea level response to alongshelf wind stress along the West Coast of North America, 1980-1984. Journal of Geophysical Research, 92, 11,761-11,788.

Hsueh, Y., 1980: Scattering of continental shelf waves by longshore variations in bottom topography. Journal of Geophysical Research, 85, 1147-1150.

Huthnance, J.M., 1975: On trapped waves over a continental shelf. Journal of Fluid Mechanics, 69, 689-704.

Huthnance, J.M., 1978: On coastal trapped waves: Analysis and numerical calculation by inverse iteration. Journal of Physical Oceanography, 8, 74-92.

Huthnance, J.M., L.A. Mysak and D.-P. Wang, 1986: Coastal trapped waves. In Baroclinic Processes on Continental Shelves, edited by C.N.K. Mooers, American Geophysical Union, Washington D.C., pp. 1-18.

Ives, D.C. and R.M. Zacharias: Conformal mapping and orthogonal grid generation, Paper No. 87-2057, AIAA/SAE/ASME/ASEE 23rd Joint Propulsion Conference, San Diego, California, June 1987.

Killworth, P.D., 1978: Coastal upwelling and Kelvin waves with small longshore topography. Journal of Physical Oceanography, 8, 188-205.

Margenau, H. and G.M. Murphy: The Mathematics of Physics and Chemistry, 581.pp., D. Van Nostrand, New York, 1943.

Miles, J.W., 1972: Kelvin waves on oceanic boundaries. Journal of Fluid Mechanics, 55, 113127.

Mitchum, G.T. and A.J. Clarke, 1986: Evaluation of frictional, wind forced long wave theory on the West Florida shelf. Journal of Physical Oceanography, 16, 1029-1037. 
Mysak, L.A., 1980a: Recent advances in shelf wave dynamics. Reviews of Geophysics and Space Physics, 18, 211-241.

Mysak, L.A., 1980b: Topographically trapped waves. Annual Review of Fluid Mechanics, 12, $45-76$.

Mysak, L.A. and C.L. Tang, 1974: Kelvin wave propagation along an irregular coastline. Journal of Fluid Mechanics, 64, 241-261.

Orlanski, I, 1976: A simple boundary condition for unbounded hyperbolic flows. Journal of Computational Physics, 21, 251-269.

Packham, B. and W. Williams, 1968: Diffraction of Kelvin waves at a sharp bend. Journal of Fluid Mechanics, 34, 517-529.

Smith, R.L., 1978: Poleward propagating perturbations in currents and sea levels along the Peru coast. Journal of Geophysical Research, 83, 6083-6092.

Wang, D.-P., 1980: Diffraction of continental shelf waves by irregular alongshore geometry. Journal of Physical Oceanography, 10, 1187-1199.

Webster, I., 1987: Scattering of coastally trapped waves by changes in continental shelf width. Journal of Physical Oceanography, 17, 928-937.

Wilkin, J.L., A computer program for calculating frequencies and modal structures of free coastal-trapped waves, Technical Report WHOI 87-53, 50pp., Woods Hole Oceanographic Institution, 1987.

Wilkin, J.L., A computer program for generating two-dimensional orthogonal curvilinear coordinate grids, Technical Report WHOI 88- , in preparation, Woods Hole Oceanographic Institution, 1988.

Yeh, H.-C., 1975: Method of solving potential field problems with complicated geometries. Journal of Applied Physics, 46, 4431-4440. 